\title{
Absolute Steady-State Thermal Conductivity Measurements by Use of a Transient Hot-Wire System
}

Hans M. Roder, Richard A. Perkins, and Arno Laesecke

National Institute of Standards and Technology, Boulder, CO 80303, USA

hans.roder@nist.gov

richard.perkins@nist.gov

arno.laesecke@nist.gov

and

\section{Carlos A. Nieto de Castro}

Departamento de Qumica and Centro de Cincias da Universidade de Lisboa, 1700 Lisboa, Portugal

carlos.castro@fc.ul.pt
A transient hot-wire apparatus was used to measure the thermal conductivity of argon with both steady-state and transient methods. The effects of wire diameter, eccentricity of the wire in the cavity, axial conduction, and natural convection were accounted for in the analysis of the steadystate measurements. Based on measurements on argon, the relative uncertainty at the $95 \%$ level of confidence of the new steady-state measurements is $2 \%$ at low densities. Using the same hot wires, the relative uncertainty of the transient measurements is $1 \%$ at the $95 \%$ level of confidence. This is the first report of thermal conductivity measurements made by two different methods in the same apparatus. The steady-state method is shown to complement normal transient measurements at low densities, particularly for fluids where the thermophysical properties at low densities are not known with high accuracy.

Key words: argon; convection; dilute gas, hot wire instrument; steady state; thermal conductivity; transient.

Accepted: February 4, 2000

Available online: http://www.nist.gov/jres

\section{Introduction}

The transient hot-wire system has been accepted widely as the most accurate technique for measuring the thermal conductivity of fluids over a wide range of physical states removed from the critical region. However, one of the drawbacks of this system is the need for increasingly larger corrections for the finite wire diameter and the outer boundary in the limit of zero fluid density. This effectively establishes a lower limit in pressure, at approximately $1 \mathrm{MPa}$ for argon (corresponding to $28 \mathrm{~kg} \mathrm{~m}^{-3}$ in density), where the uncertainty in thermal conductivity increases dramatically. If transient measurements are made on gases below $1 \mathrm{MPa}$, the thermal conductivity is generally higher than the best theoretical estimates. Below $1 \mathrm{MPa}$, the linear region in the temperature rise vs the logarithm of time is greatly reduced or no longer exists for transient hot-wire measurements of gases. This curvature in the transient temperature rise is due to extremely large effects of the correction for finite physical properties of the wire at short times, and to the penetration of the transient thermal wave to the outer boundary at longer times, as the thermal diffusivity increases significantly in the limit of 
zero density. At low densities, the magnitudes of these corrections (comparable to the measured temperature rise itself) make it almost impossible to obtain an accurate mathematical description of the observed transient heat transfer in the hot-wire cells.

To overcome these difficulties, researchers often extrapolate measurements along an isotherm from higher densities to obtain the thermal conductivity of the dilute gas. The dilute-gas thermal-conductivity data obtained by such an extrapolation procedure have significantly more uncertainty than the data used in the extrapolation. Near the critical temperature the critical enhancement contributes significantly to the total thermal conductivity even at relatively low densities. This introduces curvature in thermal conductivity isotherms and makes the extrapolation to the dilute gas limit even more uncertain for isotherms near the critical temperature. Furthermore, the $1 \mathrm{MPa}$ restriction makes the transient hot-wire instruments inappropriate for measuring thermal conductivity in the vapor phase at temperatures where the vapor pressure is below $1 \mathrm{MPa}$. Inconsistencies in dilutegas thermal conductivities obtained by various researchers using transient hot wires have seriously weakened the credibility of the technique.

These problems increase the relative uncertainty at the level of $95 \%$ confidence of the thermal conductivity obtained with the transient hot-wire technique from $0.3 \%$ for measurements at higher densities to about $2 \%$ at the lower densities, which are the focus of the present work. This $2 \%$ relative uncertainty is comparable to the relative uncertainty of measurements obtained with accurate steady-state instruments. The largest uncertainty in steady-state measurements is due to fluid convection and this is known to decrease dramatically in the limit of zero density. Corrections to steady-state measurements actually decrease and become negligible in this dilutegas region where transient measurements encounter their most serious difficulties.

At low densities the transient mode of heat transfer occurs at extremely short real times, where the wire heat-capacity correction is still quite large. This is because the thermal diffusivity of the gas is very large at low densities. This fast approach to steady state at low gas densities is an advantage for steady-state measurements using the same wire geometry. This paper examines the possibility of using the steady-state mode of operation to obtain the thermal conductivity of the dilute gas which is consistent with the higher density data obtained with the transient mode. This would allow any hot-wire instrument to operate in a transient mode at higher densities and in a steady-state mode down to the dilute-gas limit.

Measurements at low density have been made on argon gas to test this concept. Argon was selected because the dilute-gas value can be evaluated from the secondorder Chapman-Enskog kinetic theory using the known pair-interaction potential. In addition, there are many accurate measurements of the thermal conductivity of argon available in this region using both steady-state and transient techniques. Each series of measurements was made over a wide range of applied powers and included a transient and a steady-state measurement at each power level. Both the transient and the steady-state measurements are compared with the best available predictions from kinetic theory and the other data from the literature. Agreement between measurements using both modes of operation demonstrates the validity of both techniques. Researchers with transient hot wire instruments can potentially select the optimum technique for a given fluid state.

The transient hot-wire systems at NIST are completely described in previous papers [1-4]. The apparatus for high-temperature measurements [4] was used in the present work. The transient measurements were of one-second duration, as is typical in our previous measurements. The major change was modification of the data-acquisition system to operate in a steady-state mode, at times up to $40 \mathrm{~s}$. A composite picture of the voltage rises across the Wheatstone bridge, obtained in five transient runs made at the same power level but with different experimental times, is shown in Fig. 1. Time is shown on a logarithmic scale, with the experimental times ranging from $1 \mathrm{~s}$ to $40 \mathrm{~s}$. Two linear segments of the voltage rises are clearly visible. The transient thermal conductivity is obtained from the linear portion of finite slope which is proportional to the logarithm of time, while the steady-state thermal conductivity is calculated from the horizontal portion.

In Fig. 2, a typical voltage rise for a measurement in argon gas is shown, at a pressure below $1 \mathrm{kPa}$ (mild vacuum), with an experimental duration of $1 \mathrm{~s}$. This run would normally be evaluated as a transient experiment, the thermal conductivity being obtained from the apparent linear portion between $0.05 \mathrm{~s}$ and $0.15 \mathrm{~s}$. There are two reasons to make measurements under these conditions. First, one can show that there is a sufficient section of a horizontal straight line, even at times below $1 \mathrm{~s}$, to obtain a reliable result for steady-state conditions. Secondly, it may be possible to extract values for axial conduction or end effects. In addition, this extreme example shows exactly the origin of the problem with the transient experiment at low densities. The linear region in the temperature rise vs. the logarithm of time is extremely limited, degrading the accuracy of the resulting thermal conductivity data. In other words, a valid constant slope cannot be extracted from such a curve for temperature rise. 


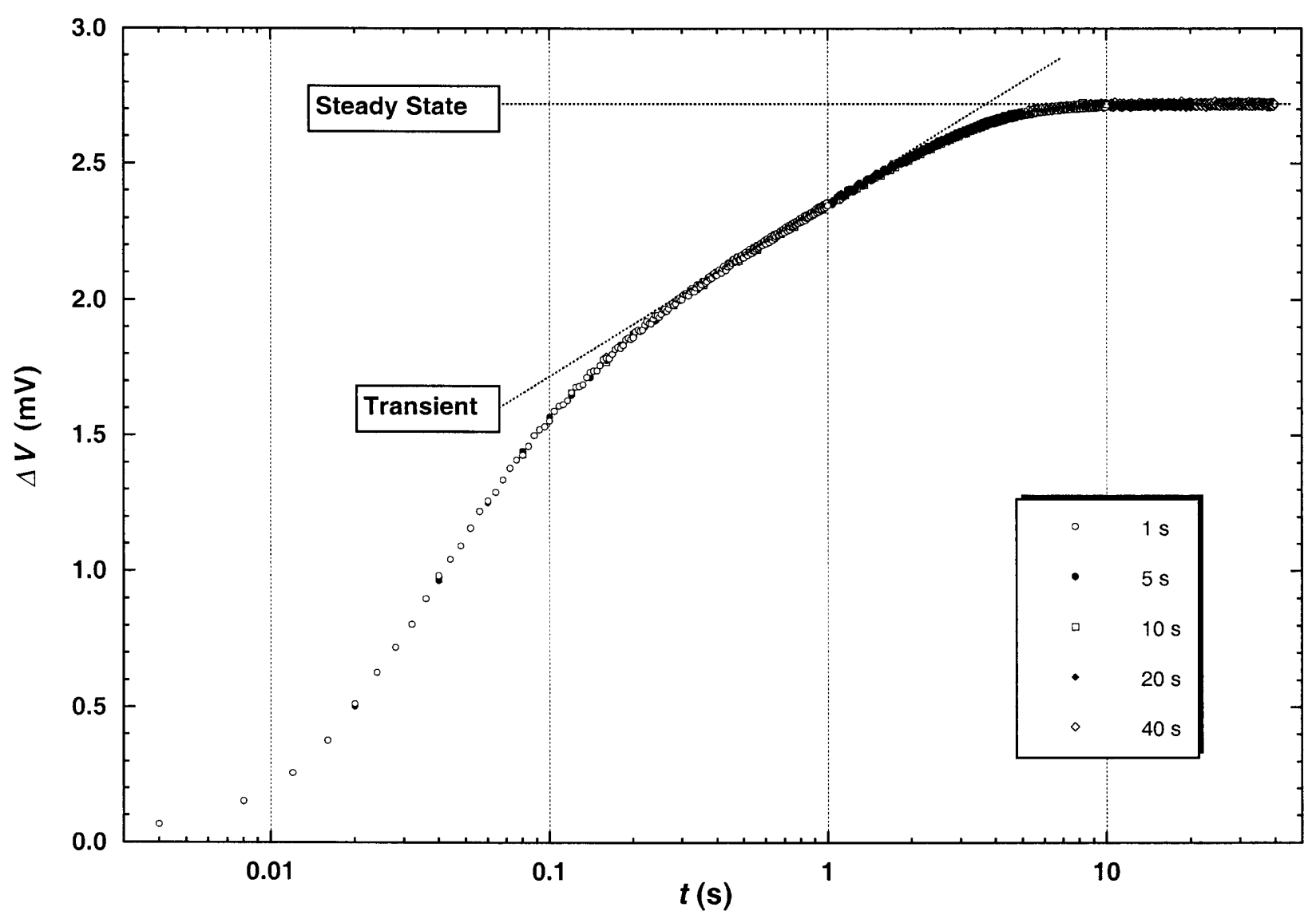

Fig. 1. A composite of bridge imbalances from five experiments with different durations for argon at $1 \mathrm{MPa}$ and $300 \mathrm{~K}$. Both the rising transient region and the horizontal steady-state region are shown. Short time curvature is due to the finite wire diameter, while curvature between the transient and steady-state regions is due to penetration of the temperature gradient to the outer cell wall.

\section{Transient Mode}

Since transient measurements are increasingly unreliable as pressure decreases below $1 \mathrm{MPa}$, all assumptions made in the application of the theory of the instrument must be carefully examined. The theory for transient hot-wire measurements is well developed [5,6], although proper application of the theory requires significant care and judgment. The hot-wire cells are designed to approximate a transient line source as closely as possible, and deviations from this model are treated as corrections to the experimental temperature rise. The ideal temperature rise $\Delta T_{\mathrm{id}}$ is given by

$$
\Delta T_{\mathrm{id}}=\frac{q}{4 \pi \lambda}\left[\ln (t)+\ln \left(\frac{4 a}{r_{0}^{2} C}\right)\right]=\Delta T_{\mathrm{W}}+\sum_{i=1}^{10} \delta T_{i}
$$

where $q$ is the power applied divided by length, $\lambda$ is the thermal conductivity of the fluid, $t$ is the elapsed time, $a=\lambda / \rho C_{p}$ is the thermal diffusivity of the fluid, $\rho$ is the mass density of the fluid, $C_{p}$ is the isobaric heat capac- ity of the fluid, $r_{0}$ is the radius of the hot wire, $C=\mathrm{e}^{\gamma}=1.781 \ldots$ is the exponential of Euler's constant, $\Delta T_{\mathrm{w}}$ is the measured temperature rise of the wire, and $\delta T_{i}$ are corrections to account for deviations from ideal line-source conduction $[5,6]$. The two most significant corrections account for the finite radius of the wire and for penetration of the fluid temperature gradient to the outer cell wall. The finite wire radius produces the short-time temperature lag relative to the ideal model, as shown in Fig. 1. Penetration of the temperature gradient to the outer wall produces the transition from the linear transient region to the steady-state conduction mode, which is also shown in Fig. 1.

It is apparent in Eq. (1) that the thermal conductivity can be found from the slope of the ideal temperature rise as a function of the logarithm of elapsed time. The thermal diffusivity can be found from the intercept of this linear function. The uncertainty of the thermal conductivity at a level of confidence of $95 \%$ is obtained from a linear fit of the ideal temperature rise data to Eq. (1) and is characterized by the parameter STAT. A STAT 


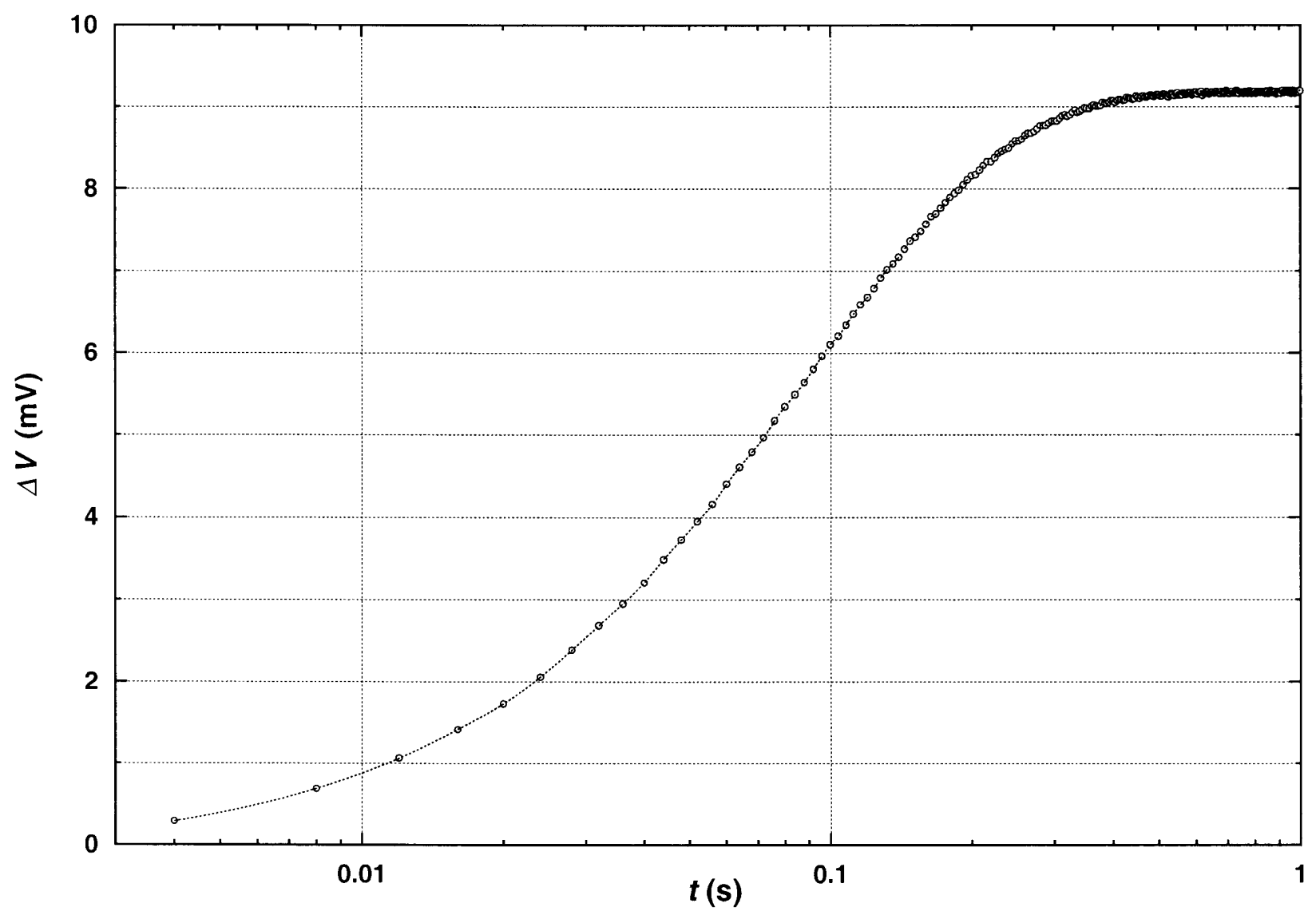

Fig. 2. Bridge imbalance at conditions close to vacuum. Here, steady-state heat transfer is reached in less than $1 \mathrm{~s}$ and the transient region is almost nonexistent.

of 0.003 , for example, corresponds to a reproducibility of $0.3 \%$ for the reported thermal conductivity. The principal corrections to the ideal model account for the finite dimensions of the wire $\delta T_{1}$, penetration of the expanding thermal wave to the outer boundary $\delta T_{2}$, and thermal radiation $\delta T_{5}$. The relative magnitude of each correction depends on the fluid properties and the elapsed time in the experiment. In this work we apply the standard corrections [5,6], including the outerboundary correction and the thermal-radiation correction for a transparent gas. The correction for the finite wire dimensions requires careful examination.

The hot wires have finite diameters and their specific heat introduces a temperature lag from the ideal line source model of Eq. (1). The temperature response of an infinitely long wire of finite radius $r_{0}$ is given [5] by

$$
=\frac{4 q \lambda a}{\pi^{3} r_{0}^{3}} \int_{0}^{\infty} \frac{\left(1-\exp \left(-a_{\mathrm{w}} u^{2} t\right)\right) \mathrm{J}_{0}(u r) \mathrm{J}_{1}\left(u r_{0}\right)}{u^{4}\left(\phi^{2}(u)+\psi^{2}(u)\right)} \mathrm{d} u,
$$

where

$$
\begin{gathered}
\phi(u)=\lambda_{\mathrm{w}} a^{1 / 2} \mathrm{~J}_{1}\left(r_{0} u\right) \mathrm{J}_{0}\left(r_{0} u \sqrt{a_{\mathrm{w}} / a}\right) \\
-\lambda a_{\mathrm{w}}^{0.5} \mathrm{~J}_{0}\left(r_{0} u\right) \mathrm{J}_{1}\left(r_{0} u \sqrt{a_{\mathrm{w}} / a}\right)
\end{gathered}
$$

and

$$
\begin{aligned}
& \psi(u)=\lambda_{\mathrm{w}} a^{1 / 2} \mathrm{~J}_{1}\left(r_{0} u\right) \mathrm{Y}_{0}\left(r_{0} u \sqrt{a_{\mathrm{w}} / a}\right) \\
& -\lambda a_{\mathrm{w}}^{1 / 2} \mathrm{~J}_{0}\left(r_{0} u\right) \mathrm{Y}_{1}\left(r_{0} u \sqrt{a_{\mathrm{w}} / a}\right),
\end{aligned}
$$

where $\mathrm{J}_{n}$ is the Bessel function of the first kind with order $n, \mathrm{Y}_{n}$ is the Bessel function of the second kind with order $n$. In Eqs. (2)-(4) the thermal conductivity of the wire is $\lambda_{\mathrm{w}}$ and the thermal diffusivity of the wire is $a_{\mathrm{w}}$. Eqs. (2)-(4) are defined for any point in the wire, and it is the volume-averaged temperature from $r=0$ to $r=r_{0}$ which is required to correct our experimental observed rises in temperature. The resulting correction, $\delta T_{1 \mathrm{f}}$, for the finite wire dimension is 


$$
\delta T_{1 \mathrm{f}}=\int_{0}^{r_{0}} \frac{2 \Delta T_{\text {full }}(r, t) r}{r_{0}{ }^{2}} \mathrm{~d} r-\Delta T_{\text {id }} .
$$

Although it is fairly simple to implement this full solution to correct the experimental temperature rise, it is the first-order expansion of this solution which has been recommended because of its simplicity $[5,6]$. This first-order expansion for a bare wire is $[5,6]$

$$
\begin{gathered}
\delta T_{1}=\frac{q}{4 \pi \lambda}\left[\frac{r_{0}^{2}\left(\left(\rho C_{p}\right)_{\mathrm{w}}-\rho C_{p}\right)}{2 \lambda t} \ln \left(\frac{4 a t}{r_{0}^{2} C}\right)\right. \\
\left.-\frac{r_{0}^{2}}{2 a t}+\frac{r_{0}^{2}}{4 a_{\mathrm{w}} t}-\frac{\lambda}{2 \lambda_{\mathrm{w}}}\right] .
\end{gathered}
$$

Before an approximation such as Eq. (6) can be used, the truncation error for the case of our relatively large $12.7 \mu \mathrm{m}$ platinum hot wire must be evaluated. Figure 3 shows the full solution for the transient temperature rise [Eqs. (2)-(5)], along with the first- [Eq. (6)] and secondorder approximations [7], for the case of a $12.7 \mu \mathrm{m}$ platinum hot wire in argon gas at $300 \mathrm{~K}$ and $0.1 \mathrm{MPa}$. It is apparent in Fig. 3 that the truncation error is quite large for dilute gases, so the first-order approximation of
Eq. (6) cannot be used to correct the data. Both the magnitude of the correction for physical properties of the finite wire and the associated truncation error can be minimized by using thinner hot wires. Table 1 shows how truncation corrections depend on the wire diameter for argon gas at $300 \mathrm{~K}$ and $0.1 \mathrm{MPa}$. Also included in Table 1 are the relative uncertainties in thermal conductivity resulting from a relative uncertainty of $10 \%$ in the thermal diffusivity of the fluid.

In the limit of zero density, the transient thermal wave will penetrate to the outer boundary. The outerboundary correction, which accounts for penetration of the transient heat pulse $[5,6]$, is given by

$\delta T_{2}=\frac{q}{4 \pi \lambda}\left[\ln \left(\frac{4 a t}{r_{\mathrm{b}}^{2} C}\right)+\sum_{v=1}^{\infty} \exp \left(\frac{-g_{v} a t}{r_{\mathrm{b}}^{2}}\right)\left[\pi \mathrm{Y}_{0}\left(g_{v}\right)\right]^{2}\right]$

where $g_{\nu}$ are the roots of $\mathbf{J}_{0}\left(g_{\nu}\right)=0$ and $r_{\mathrm{b}}$ is the radius of the outer boundary. In the limit of infinite time, Eq. (7) approaches the steady-state solution given below.

The final corrections, which must be considered because of their increasing significance at low densities, account for compression work $\delta T_{3}$ and radial convection $\delta T_{4}$. A recent analysis by Assael et al. [8] concludes that $\delta T_{3}$ and $\delta T_{4}$ must be considered simultaneously and that

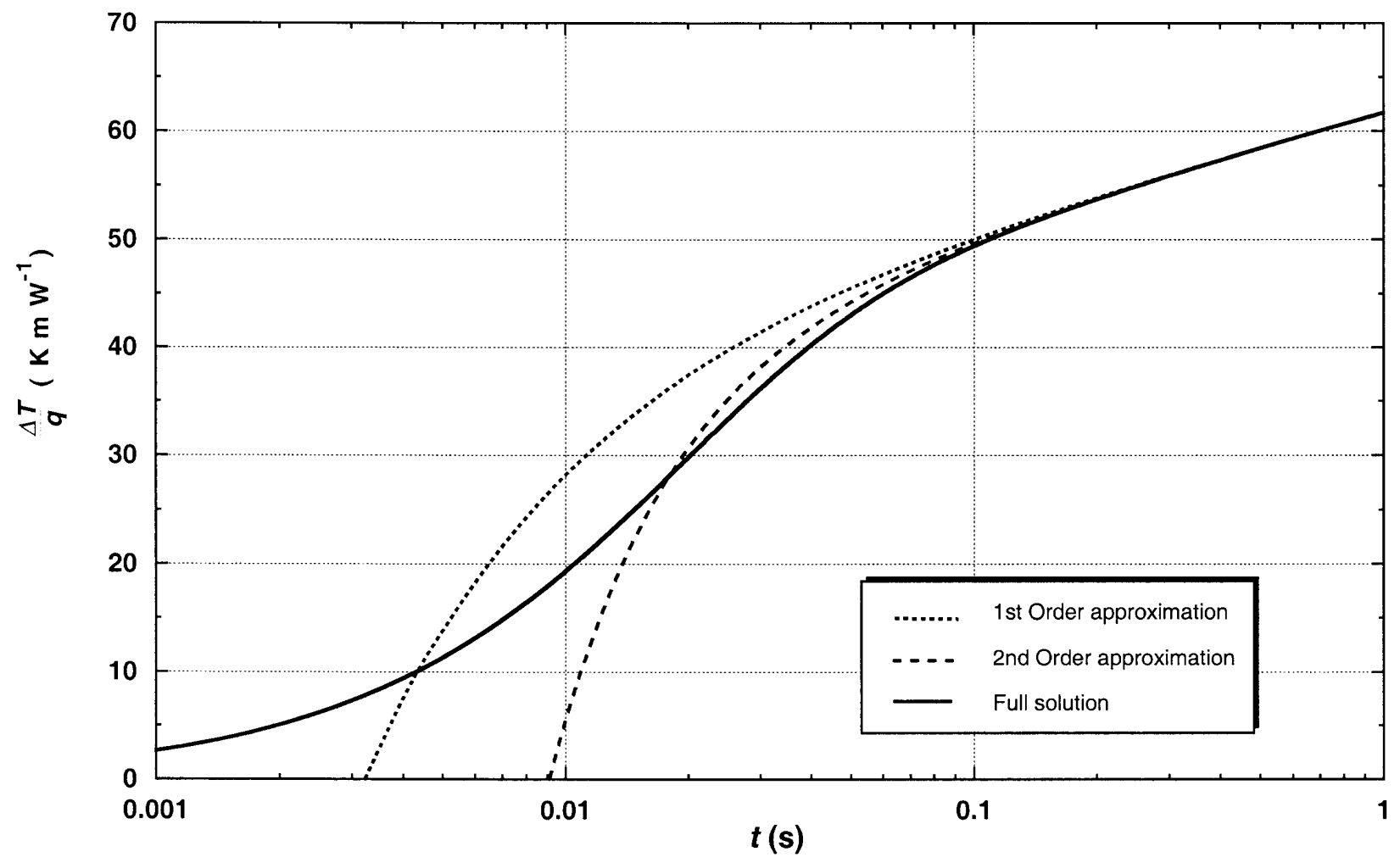

Fig. 3. Calculated temperature rise for finite physical properties of the wire for argon gas at $300 \mathrm{~K}$ and $0.1 \mathrm{MPa}$ using first-order and second-order approximations as well as the full integral solution. 
Table 1. Uncertainties due to corrections for finite wire diameter for argon gas at $300 \mathrm{~K}$ and $0.1 \mathrm{MPa}$. First, differences are examined between the actual heat transfer from a finite diameter wire and the first and second order series approximations of this solution for various wire diameters. Second, the effect of a $10 \%$ error in the thermal diffusivity $a$ used for finite wire diameter correction is calculated for various wire diameters. This correction can introduce significant errors in thermal conductivity measurements with large wires if the thermal diffusivity or wire diameter is not well known.

\begin{tabular}{|c|c|c|c|c|c|c|}
\hline Fluid & $\begin{array}{l}\text { Wire diameter } \\
\qquad(\mu \mathrm{m})\end{array}$ & Ideal slope & $\begin{array}{l}\text { Slope after } 1 \text { st } \\
\text { order correction } \\
\text { is applied }\end{array}$ & $\begin{array}{l}\text { Slope after } 2 \mathrm{nd} \\
\text { order correction } \\
\text { is applied }\end{array}$ & $\begin{array}{c}\text { Relative } \\
\text { deviation in } \\
\lambda \text { after } 1 \text { st order } \\
\text { correction }\end{array}$ & $\begin{array}{c}\text { Relative } \\
\text { deviation in } \\
\lambda \text { after } 2 \text { nd } \\
\text { order correction }\end{array}$ \\
\hline
\end{tabular}

\begin{tabular}{|c|c|c|c|c|c|c|}
\hline \multicolumn{7}{|c|}{ Mathematical approximation errors } \\
\hline Argon & 7.0 & 4.445669 & 4.451398 & 4.446015 & $-0.12 \%$ & $-0.01 \%$ \\
\hline Argon & 12.5 & 4.445669 & 4.504379 & 4.456487 & $-1.32 \%$ & $-0.24 \%$ \\
\hline Argon & 25.0 & 4.445669 & 5.508840 & 4.952375 & $-23.91 \%$ & $-11.40 \%$ \\
\hline Fluid & $\begin{array}{l}\text { Wire diameter } \\
\qquad(\mu \mathrm{m})\end{array}$ & Ideal slope & $\begin{array}{l}\text { Apparent slope } \\
\text { with a }-10 \% \\
\text { error in thermal } \\
\text { diffusivity }\end{array}$ & $\begin{array}{l}\text { Apparent slope } \\
\text { with a }+10 \% \\
\text { error in thermal } \\
\text { diffusivity }\end{array}$ & $\begin{array}{c}\text { Relative } \\
\text { deviation in } \\
\lambda \text { with a }-10 \% \\
\text { error in thermal } \\
\text { diffusivity }\end{array}$ & $\begin{array}{c}\text { Relative } \\
\text { deviation in } \\
\lambda \text { with a }+10 \% \\
\text { error in thermal } \\
\text { diffusivity }\end{array}$ \\
\hline
\end{tabular}

Thermal conductivity uncertainty associated with $10 \%$ uncertainty in fluid thermal diffusivity

\begin{tabular}{lrrrrrr}
\hline Argon & 7.0 & 4.445669 & 4.444565 & 4.446668 & $-0.03 \%$ & $+0.02 \%$ \\
Argon & 12.5 & 4.445669 & 4.441077 & 4.449814 & $-0.10 \%$ & $+0.09 \%$ \\
Argon & 25.0 & 4.445669 & 4.398395 & 4.487762 & $-1.06 \%$ & $+0.94 \%$ \\
\hline
\end{tabular}

the previous analysis of Healy et al. [5] is in error. Based on the work of Assael et al. [8] we have set both $\delta T_{3}$ and $\delta T_{4}$ equal to zero in the present analysis.

At low densities, the thermal diffusivity of the fluid increases almost linearly with inverse pressure. Large corrections to the experimental temperature rise are required for both heat-capacity and outer-boundary effects because of this large thermal diffusivity. Any uncertainties in the wire diameter and the fluid properties used in these corrections become increasingly important at low densities. The full heat-capacity correction must be used to correct the measured temperature rises at low densities since this correction is so significant. The measurements at low density must be carefully examined to verify that the thermal wave has not reached the outer boundary since the thermal diffusivity increases so dramatically in this region.

Transient results obtained by use of the traditional corrections employed in earlier work $[5,6]$ and the revised corrections as discussed above are shown for argon at $300 \mathrm{~K}$ in Fig. 4. The differences are primarily due to setting the compression work $\delta T_{3}$ equal to zero [8], use of the full heat-capacity correction of Eqs. (2)-(5), and careful restriction of the regression limits to exclude times where the outer-boundary correction $\delta T_{2}$ is significant. The results for all power levels were averaged at each pressure level in Fig. 4. It can be seen that the results at low densities are more linear in terms of density with the revised corrections (hook due to increasing contributions from the outer boundary correction), while the results at the higher densities are not changed appreciably. This linear dependence on density is expected from the kinetic theory of low-density gases.

\section{Steady-State Mode}

The working equation for the steady-state mode is based on a different solution of Fourier's law but the geometry is still that of concentric cylinders. The solution can be found in standard texts for the case of constant thermal conductivity (see, for example, Reference [9], page 114). This equation can be solved for the thermal conductivity of the fluid $\lambda$;

$$
\lambda=\frac{q \ln \left(\frac{r_{2}}{r_{1}}\right)}{2 \pi\left(T_{1}-T_{2}\right)},
$$

where $q$ is the applied power divided by length, $r_{2}$ is the internal radius of the outer cylinder, $r_{1}$ is the external radius of the inner cylinder (hot wire), and $\Delta T=\left(T_{1}-T_{2}\right)$ is the measured temperature difference between the hot wire and its surrounding cavity. 


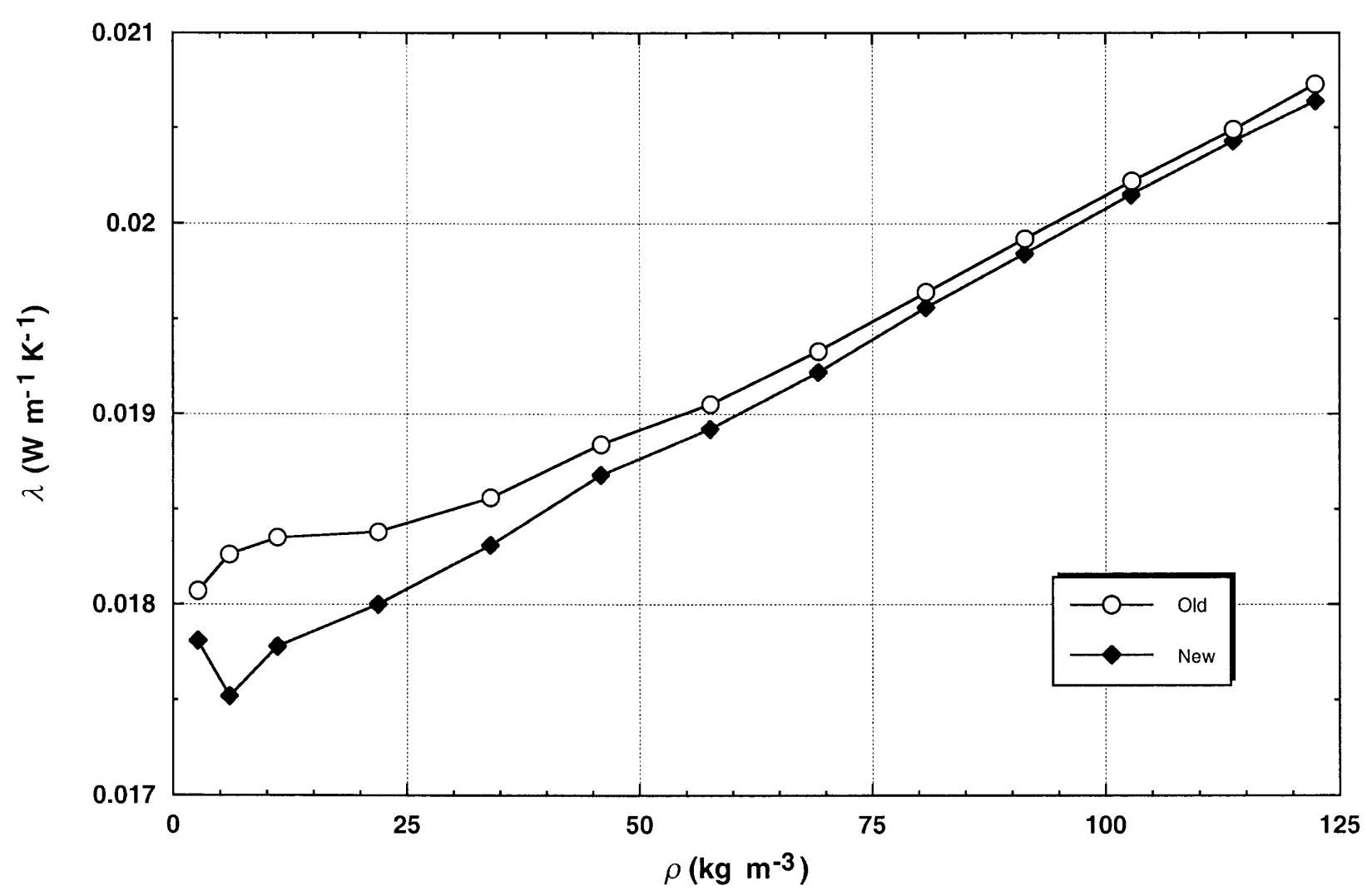

Fig. 4. Transient results for argon at $300 \mathrm{~K}$ with the corrections applied in earlier papers as well as the revised corrections proposed herein. Results were averaged at each pressure.

For the concentric-cylinder geometry described above the total heat flux divided by length, $q$, remains constant and is not a function of the radial position. Assuming that the thermal conductivity is a linear function of temperature, such that $\lambda=\lambda_{0}\left(1+b_{\lambda} T\right)$, it can be shown that the measured thermal conductivity is given by $\lambda=\lambda_{0}\left(1+b_{\lambda}\left(T_{1}+T_{2}\right) / 2\right)$. Thus, the thermal conductivity that is measured corresponds to the value at the mean temperature of the inner and outer cylinders, where

$$
\bar{T}=\left(T_{1}+T_{2}\right) / 2
$$

This assumption of a linear temperature dependence for the thermal conductivity is valid for experiments with small temperature rises. The density of the fluid assigned to the measured thermal conductivity is taken from an equation of state [10] using the temperature from Eq. (9) and the experimentally measured pressure.

Equation (8) assumes that the dimensions of the wire and cavity are well known and that the wire is perfectly concentric with the outer cylindrical cavity. The diameter of our wire is $13.14 \mu \mathrm{m}$ and it is known with a relative uncertainty of $0.5 \%$ at a level of confidence of
$95 \%$. This uncertainty contributes a component of relative uncertainty of $0.07 \%$ to the uncertainty of the measured thermal conductivity. Since it is nearly impossible to keep the wire perfectly concentric with the outer cavity, the uncertainty associated with the eccentricity of the wire with respect to the cavity must be assessed. For a wire that is eccentric, the thermal conductivity is given by

$$
\begin{gathered}
\lambda=\frac{q}{2 \pi\left(T_{1}-T_{2}\right)} \\
\ln \left[\frac{\sqrt{\left(r_{2}+r_{1}\right)^{2}-b^{2}}+\sqrt{\left(r_{2}-r_{1}\right)^{2}-b^{2}}}{\sqrt{\left(r_{2}+r_{1}\right)^{2}-b^{2}}-\sqrt{\left(r_{2}-r_{1}\right)^{2}-b^{2}}}\right],
\end{gathered}
$$

where $b$ is the distance between the wire's axis and the axis of the outer cylinder. The eccentricity correction is shown in Fig. 5 for a $12.7 \mu \mathrm{m}$ diameter wire in a $9 \mathrm{~mm}$ diameter cavity. The hot wires in the present cell are concentric with the cavity within $0.5 \mathrm{~mm}$, so it is apparent from Fig. 5 that the relative uncertainty is about $0.2 \%$ due to misalignment of the wire in the cavity. The combined relative uncertainty, due to both the diameter and eccentricity of the wire is $0.3 \%$ in the measured thermal conductivity. 


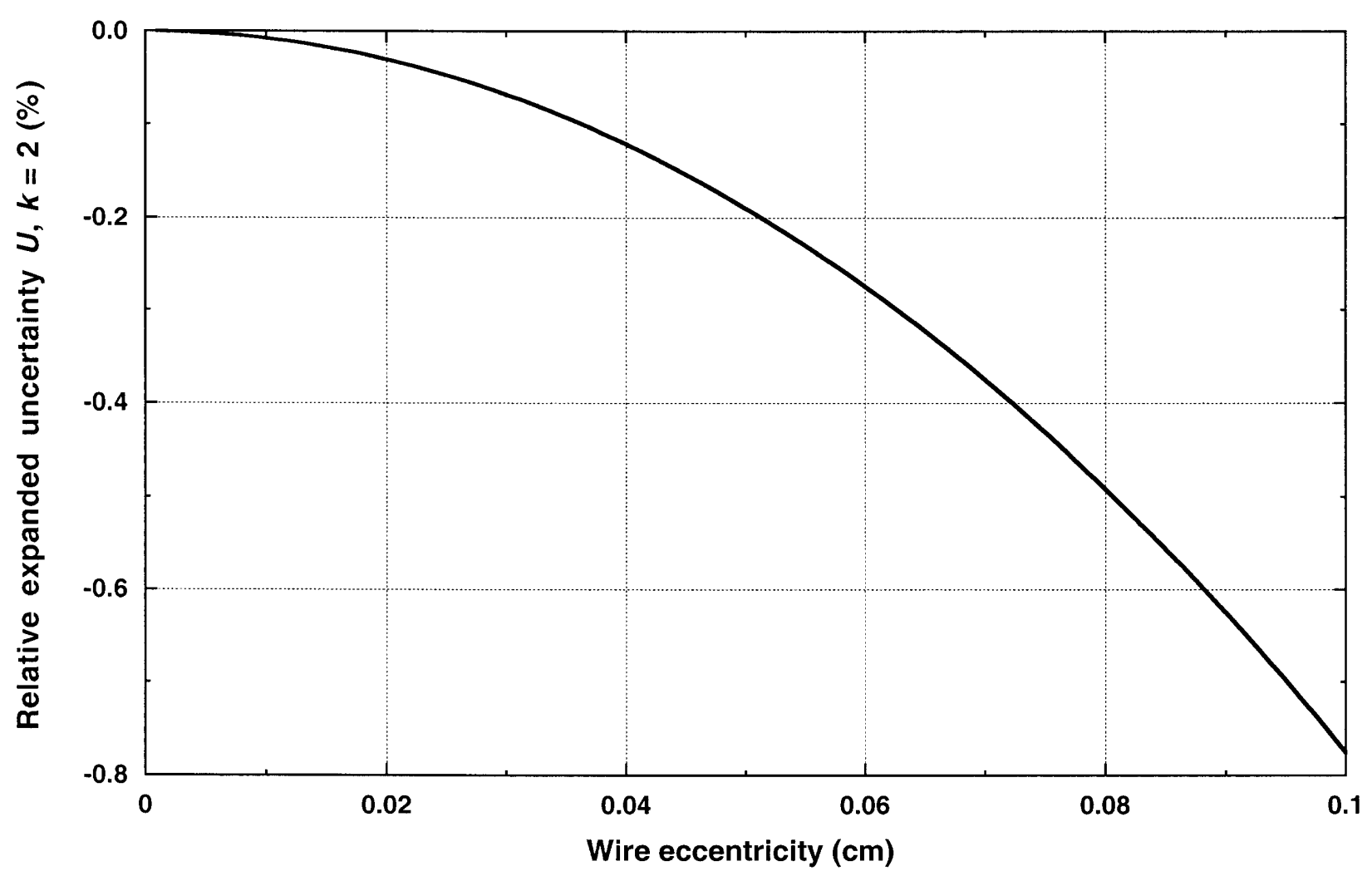

Fig. 5. Error estimate for steady-state results due to the eccentricity of the hot wire. The thermal conductivity measurement is not sensitive to eccentricity for hot wires with small diameters.

While $40 \mathrm{~s}$ may seem to be a very short time in comparison to normal steady-state measurements, it still allows the very small wires used in transient hot-wire systems to equilibrate in the gas phase. The time of the steady-state experiments is restricted to $40 \mathrm{~s}$ since the temperature of the cell wall $T_{1}$ is assumed to be the initial cell temperature. Both transient and steady-state measurements of thermal conductivity should be made at several power levels. The thermal conductivity should be valid and free of convection if a plot of the measured values of thermal conductivity as a function of applied power is constant. The present measurements are made over a large range of applied powers, and the powers of the transient measurements overlap those used for the steady-state measurements as much as possible.

\section{Data Reduction}

Three isotherms were measured for gaseous argon at $300 \mathrm{~K}, 320 \mathrm{~K}$, and $340 \mathrm{~K}$. There were 13 to 14 different pressure levels covering a density range from $120 \mathrm{~kg}$ $\mathrm{m}^{-3}$ down to $2.4 \mathrm{~kg} \mathrm{~m}^{-3}$. At each pressure level, experimental results were collected at 7 to 11 different applied powers. Transient measurements were made for an ex- perimental time of $1 \mathrm{~s}$, while for the steady-state measurements the total elapsed time used was $40 \mathrm{~s}$. In either case, 250 measurements of the bridge imbalance voltage were obtained. To elucidate the end effects in the experiment, a special series of runs were made using an additional digital voltmeter to measure directly across the long hot wire, the short hot wire, and the bridge. Finally, an abbreviated set of measurements was made for pressures below $1 \mathrm{kPa}$. In all, we made 883 measurements. The temperatures were measured on the International Practical Temperature Scale of 1968 (IPTS 68) but the effect of converting the temperatures to the International Temperature Scale of 1990 (ITS 90) on the reported thermal conductivity is less than $1 \mu \mathrm{W} \mathrm{m}{ }^{-1}$ $\mathrm{K}^{-1}$.

The steady-state measurements required the development of a new data-analysis procedure. The rises in steady-state voltage as a function of time were always examined to select reliable measurements. Five typical profiles of the bridge imbalance, used to select the appropriate range of power levels, are shown in Fig. 6. Trace b in Fig. 6 is considered reliable since it is nearly horizontal after $20 \mathrm{~s}$. In trace a, the power level is too low, so electronic noise is significant in the imbalance voltages. In traces $\mathrm{c}, \mathrm{d}$, and e the power levels are too large, so convection makes a visible contribution. 


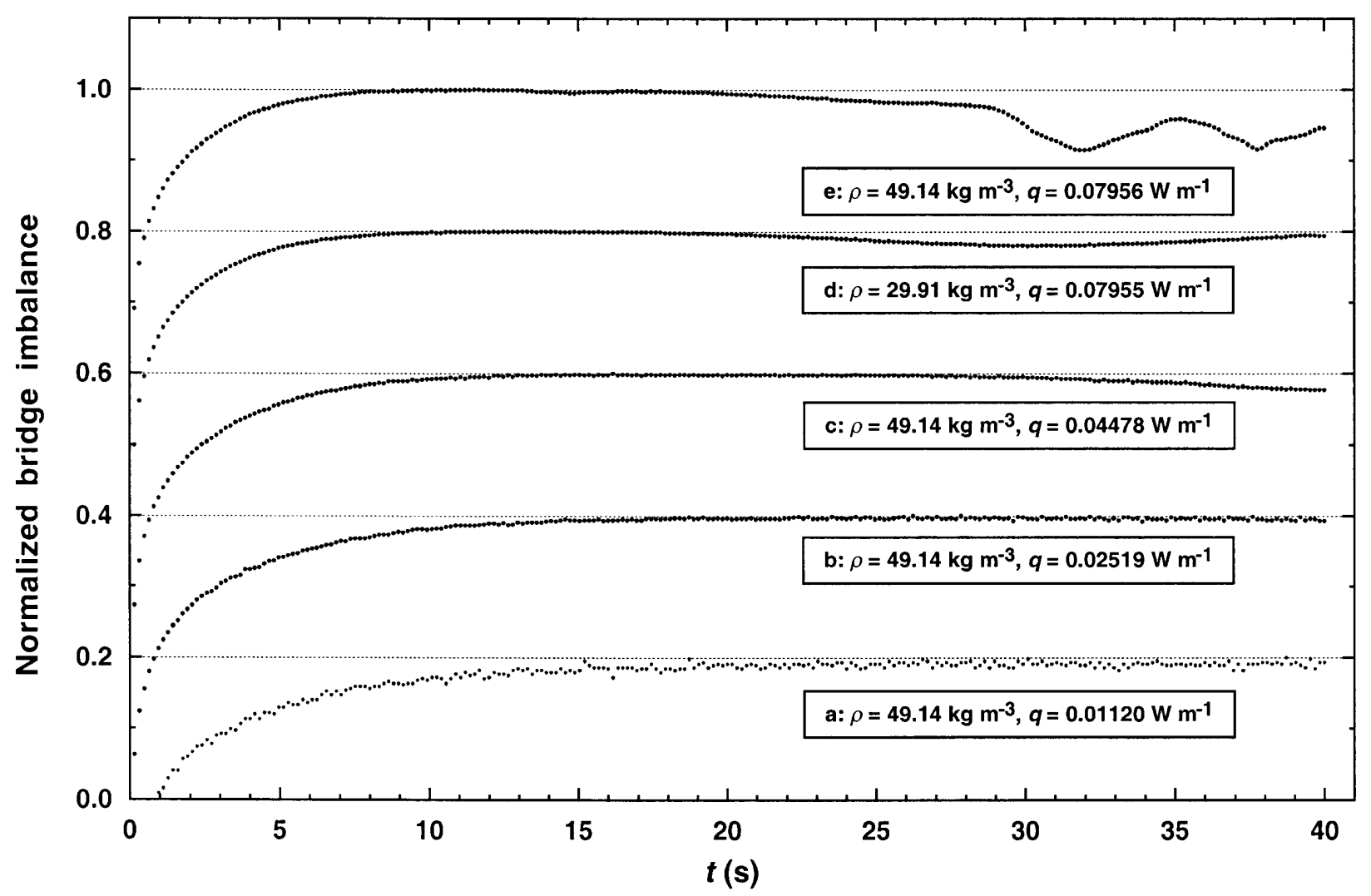

Fig. 6. Typical voltage-rise profiles for steady-state measurements at $T=340 \mathrm{~K}$. The increase in convection with the increase of applied power is shown in curves a through $\mathrm{c}$. The increase in convection with increase in fluid density is shown in curves $\mathrm{d}$ and $\mathrm{e}$.

The next step was to determine the experimental temperature rise $\Delta T$. The experimental voltage rises were averaged over a time interval where they were nearly constant. To find the optimum time to begin the averaging, the last 50 bridge imbalance voltages were averaged to find a reference imbalance voltage. The actual average, $V_{\text {ave }}$, is obtained by averaging the points from the first voltage, which is $0.5 \%$ below this reference imbalance voltage up to the final data point. Solving the bridge equation with $V_{\text {ave }}$ yields the change in resistance in the variable arms of the bridge. The resistance change is finally converted into $\Delta T$ using the calibration of the wire resistance as a function of temperature. The maximum and minimum values of the voltage rises were also obtained over the range averaged. The difference between them was expressed as a percentage of $V_{\text {ave }}$, and is designated by the parameter TBAND. TBAND is a direct measure of the precision in $\triangle T$ at the level of 3 standard deviations. In Fig. 7, the values of TBAND are plotted for all of the steady-state measurements made near 320 $\mathrm{K}$. A final selection of valid measurements was made by rejecting all points with a TBAND larger than $2 \%$. This is equivalent to rejecting those points that have voltage traces similar to traces c, d, and e in Fig. 6.
A correction for radiation was also applied to $\Delta T$. The radiation correction for transparent fluids $\delta T_{5}$ was used as given in Ref. [4]. The maximum effect of this correction was $0.13 \%$ at $340 \mathrm{~K}$. Additional corrections have been considered by other authors for steady-state hotwire systems (see for example Refs. [9,11]). These include corrections for temperature jump, end conduction in the wire, lead-wire conduction, and temperature rise in the outer wall. The temperature-jump correction does not apply because the present pressures are not low enough. The correction for end conduction in the wire and the lead-wire correction were found to be negligible in our experiments because a bridge with a compensating hot wire was used. A special series of measurements was made to determine the size of the end effects by directly measuring the temperature rise of each wire. Temperature gradients in the outer wall were considered negligible for our thick-walled pressure vessel. The primary platinum resistance thermometer (PRT) was mounted on the outside of the pressure vessel. The temperature $T_{\text {ref }}$ of the PRT increased by about $30 \mathrm{mK}$ for a series of measurements at a single pressure level. The temperature of the long hot wire, which is inside the cell, increased by a nearly identical amount. Since a 


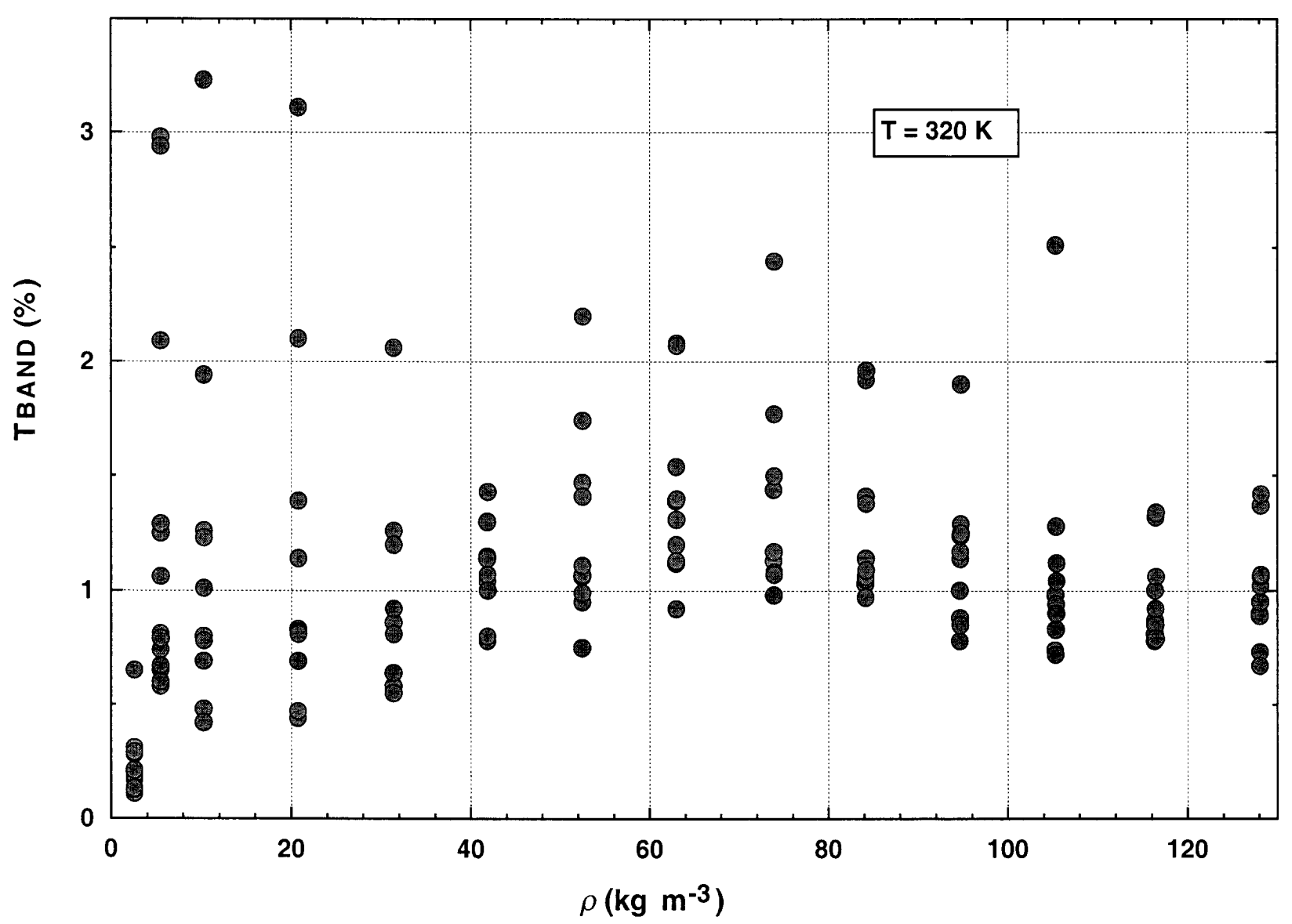

Fig. 7. Temperature uncertainty, TBAND, for the $320 \mathrm{~K}$ isotherm of argon. Data with TBAND values greater that $2 \%$ were considered invalid due to free-convection contributions.

measurement series normally consisted of about 20 measurements at time intervals $1 \mathrm{~min}$ apart, an average change of $1 \mathrm{mK}$ per measurement was negligible in comparison to the measured $\Delta T$, which was typically from $1 \mathrm{~K}$ to $4 \mathrm{~K}$.

\subsection{Free Convection}

Convection has always been a problem in measuring thermal conductivity; its onset has been associated with the Rayleigh number. One of the major advantages of the transient method is the ability to detect and avoid contributions from convection. The present measurements using the steady-state mode also show the evolution of convection very clearly. Figure 8 shows the deviations between the steady-state measurements near 320 $\mathrm{K}$, calculated before application of the correction for convection, and the thermal conductivity surface for argon [12]. Since the diameter of the hot wires is comparable to the boundary-layer thickness for heat transfer, the standard engineering models for vertical flat plates are not applicable, and so an empirical expression was developed for the thin-wire geometry.

The dimensionless Rayleigh number is commonly used to characterize the onset of free convection. For a concentric-cylinder geometry, the Rayleigh number is given by

$$
R a=\frac{g_{\mathrm{c}}\left(r_{\mathrm{b}}-r_{0}\right)^{3}\left(\frac{\partial \rho}{\partial T}\right)_{P} \Delta T}{\eta a}
$$

where $g_{\mathrm{c}}$ is the local acceleration of free fall, and $\eta$ is the fluid viscosity. The correction for natural or free convection was obtained from two equations given by Le Neindre and Tufeu [13] for a concentric-cylinder apparatus:

$$
q=q_{\text {meas }}-q_{\mathrm{c}}
$$

and

$$
\frac{q_{\mathrm{c}}}{q}=\left(\frac{d}{720 l}\right) R a=K \cdot R a,
$$




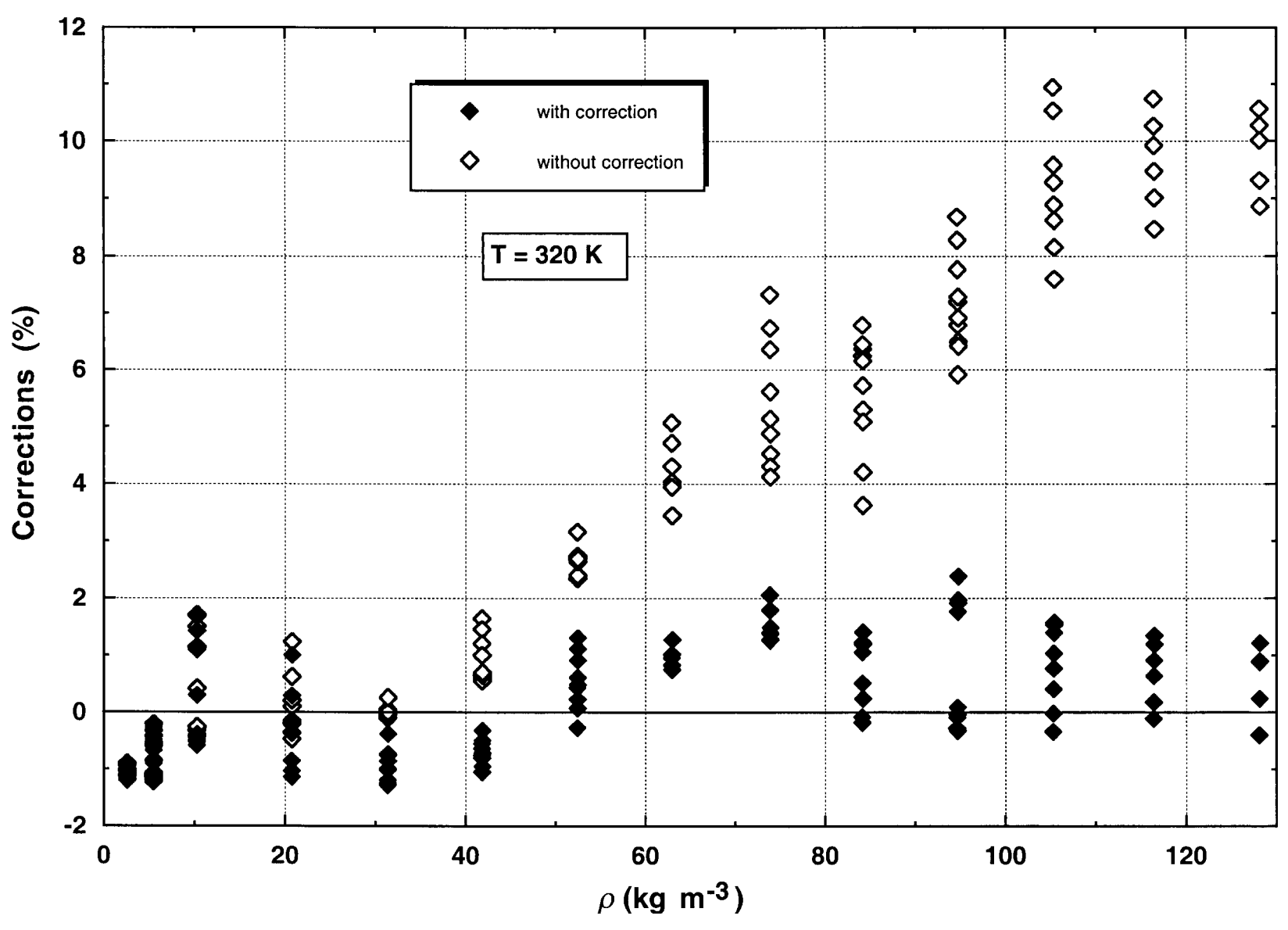

Fig. 8. Correction of steady-state results for free convection along the $320 \mathrm{~K}$ isotherm. The baseline is the correlation of Younglove and Hanley [12].

where $q$ is the applied power divided by length of Eq. (1), $q_{\text {meas }}$ is the experimental heat flow determined from the measured voltage and current, $q_{\mathrm{c}}$ is the heat transfer by natural convection, $K$ is a numerical apparatus constant, and $R a$ is the Rayleigh number. Le Neindre and Tufeu use a numerical constant of 720 , but also values of $d$, the thickness of the fluid layer, and $l$, the length of the internal cylinder. For our system, both $d$ and $l$ are constant and our ratio of $d / l$ is much larger than that of Le Neindre and Tufeu. Since the aspect ratio $d / l$ is constant in our apparatus, it is incorporated into the experimentally determined apparatus constant $K$ for our hot-wire cell. Equations (12) and (13) together give

$$
\frac{q}{q_{\text {meas }}}=\frac{1}{1+K \cdot R a}
$$

Next, Eq. (8) is applied twice, once for uncorrected conditions and once for corrected conditions. Forming a ratio we can solve for the corrected thermal conductivity

$$
\lambda_{\text {corr }}=\left(\frac{q}{q_{\text {meas }}}\right) \lambda_{\text {meas }},
$$

or with the use of Eq. (14),

$$
\lambda_{\text {corr }}=\frac{1}{(1+K \cdot R a)} \lambda_{\text {meas }} .
$$

The best value for $K$ in Eq. (16) was $1.8435 \times 10^{-6}$ and was obtained by comparing the experimental points for each isotherm against a parabolic fit of the companion transient measurements. This procedure is justified because the deviations of the companion transient measurements from the thermal conductivity surface of argon are less than $1 \%$ [12]. After applying this correction for free convection, Eq. (16), to all of the steady-state measurements, the resulting deviations are plotted in Fig. 8 for the $320 \mathrm{~K}$ isotherm. Figure 8 contains three different regions. For densities from 0 to 40 $\mathrm{kg} \mathrm{m}^{-3}$, convection contributes very little to the measured conductivity, i.e., the corrections for convection 
are less than $1 \%$. In this region, the Rayleigh numbers range from near 0 to 17000 . For densities between 40 $\mathrm{kg} \mathrm{m}^{-3}$ and $80 \mathrm{~kg} \mathrm{~m}^{-3}$ the corrections given by Eq. (16) gradually increase to about $5 \%$, while the Rayleigh numbers range up to 65000 . The correction is highly successful, as the band or range of measured values at each pressure level decreases. For steady-state thermal conductivities at densities above about $80 \mathrm{~kg} \mathrm{~m}^{-3}$, for which some of the Rayleigh numbers range well above 65000 , the corrections for simple natural convection increase to about $12 \%$. However, the uncertainty bands associated with the uncorrected thermal conductivities no longer decrease. The measured voltage rises suggest that there is flow turbulence in the cells at the larger power levels. This could be along either the short hot wire, the long hot wire, or both. Measurements associated with Rayleigh numbers greater than 70000 have to be rejected, and are omitted from Fig. 8.

\subsection{Results}

The transient measurements for all three isotherms are given in Table 2. All of the transient points with a
STAT greater than 0.003 were eliminated; thus, 98 points remain at a nominal temperature of $300 \mathrm{~K}, 105$ points remain at $320 \mathrm{~K}$, and 102 points remain at $340 \mathrm{~K}$. Figure 9 shows the deviations between all transient points and the thermal-conductivity surface of argon [12]. Based on Fig. 9 it can be concluded that, over the range of densities shown here, the deviations fit within a band of $\pm 1 \%$. The problem with the transient measurements at low densities shows up clearly as a systematic deviation. However, as shown later, this systematic deviation must be ascribed to a difference in the dilute gas $\lambda_{0}$ values used for the surface [12].

The steady-state measurements are listed in Table 3. As explained before, all steady-state results with TBAND greater than $2 \%$ and all points with Rayleigh numbers greater than 70000 were not considered. Thus, there are 104 points at a nominal temperature of $300 \mathrm{~K}$, 120 points at $320 \mathrm{~K}$, and 119 points at $340 \mathrm{~K}$. The deviations between all steady-state points and the thermal-conductivity surface of argon [12] are plotted in Fig. 10. It can be concluded from Fig. 10 that over the range of densities shown, the deviations fit within a band of $\pm 2 \%$ at a level of confidence of $95 \%$. The

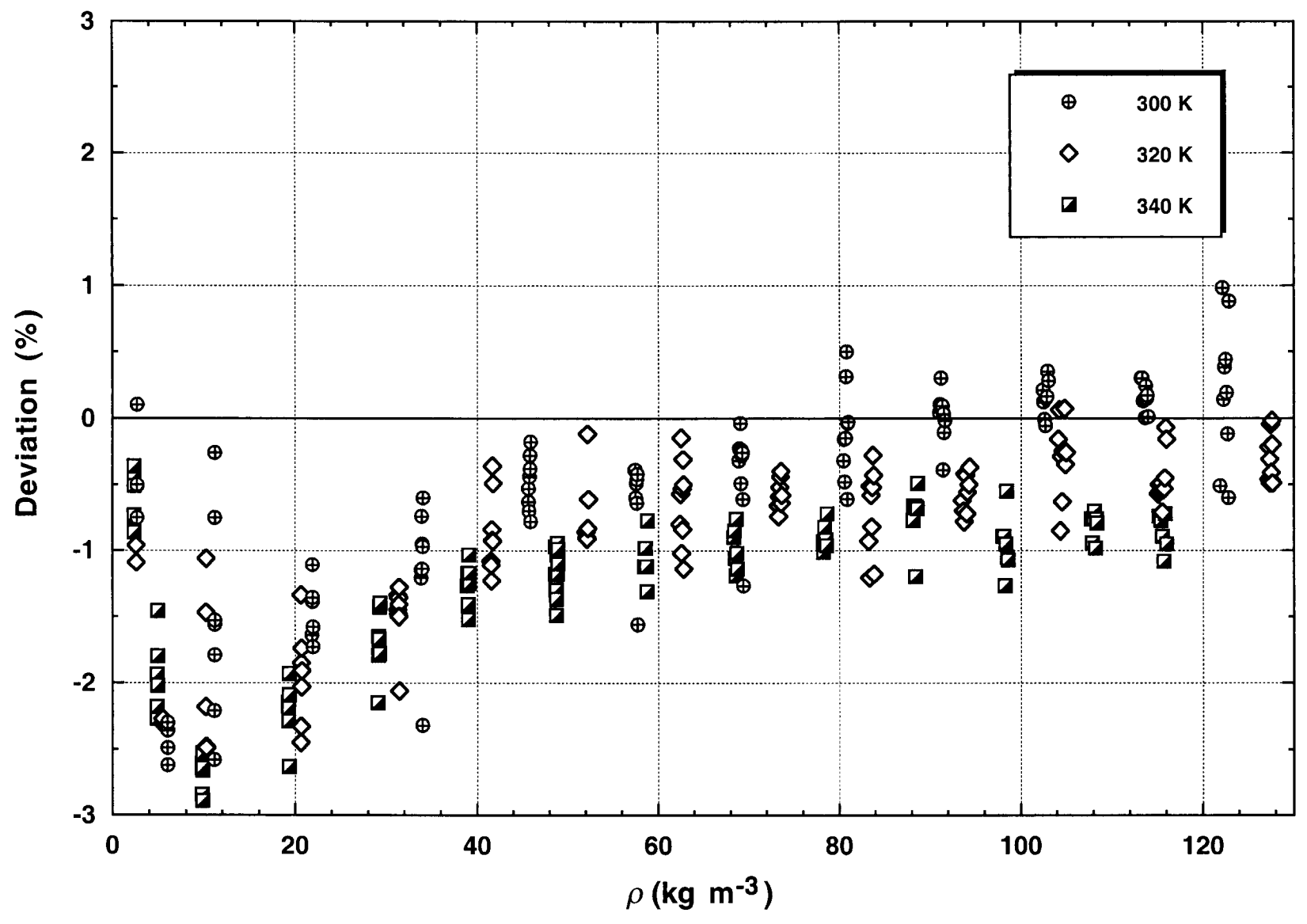

Fig. 9. Transient thermal conductivity results for argon. The baseline is the correlation of Younglove and Hanley [12]. 
Table 2. Thermal conductivity of argon, transient method

\begin{tabular}{|c|c|c|c|c|c|c|c|}
\hline $\begin{array}{c}\text { Point } \\
\text { no. }\end{array}$ & $\begin{array}{c}p \\
(\mathrm{MPa})\end{array}$ & $\begin{array}{c}\rho \\
\left(\mathrm{kg} \mathrm{m}^{-3}\right)\end{array}$ & $\begin{array}{l}T_{\exp } \\
(\mathrm{K})\end{array}$ & $\begin{array}{c}\lambda_{\exp } \\
\left(\mathrm{W} \mathrm{m}^{-1} \mathrm{~K}^{-1}\right)\end{array}$ & STAT & $\begin{array}{l}\text { Relative } \\
\text { dev. }(\%)\end{array}$ & $\begin{array}{c}q \\
\left(\mathrm{~W} \mathrm{~m}^{-1}\right)\end{array}$ \\
\hline \multicolumn{8}{|c|}{ Nominal temperature $300 \mathrm{~K}$} \\
\hline 3001 & 7.4440 & 122.916 & 301.662 & 0.02092 & 0.002 & 0.88 & 0.03941 \\
\hline 3002 & 7.4441 & 122.832 & 301.836 & 0.02062 & 0.003 & -0.60 & 0.04485 \\
\hline 3003 & 7.4440 & 122.736 & 302.022 & 0.02073 & 0.001 & -0.12 & 0.05063 \\
\hline 3004 & 7.4441 & 122.636 & 302.231 & 0.02080 & 0.001 & 0.19 & 0.05676 \\
\hline 3005 & 7.4442 & 122.525 & 302.454 & 0.02086 & 0.001 & 0.44 & 0.06324 \\
\hline 3006 & 7.4438 & 122.409 & 302.682 & 0.02086 & 0.001 & 0.38 & 0.07007 \\
\hline 3007 & 7.4440 & 122.297 & 302.914 & 0.02082 & 0.001 & 0.14 & 0.07725 \\
\hline 3008 & 7.4441 & 122.169 & 303.178 & 0.02101 & 0.001 & 0.98 & 0.08479 \\
\hline 3010 & 7.4436 & 121.893 & 303.728 & 0.02072 & 0.001 & -0.51 & 0.10090 \\
\hline 3022 & 6.9077 & 113.988 & 301.325 & 0.02049 & 0.003 & 0.01 & 0.02960 \\
\hline 3023 & 6.9078 & 113.916 & 301.488 & 0.02053 & 0.003 & 0.17 & 0.03433 \\
\hline 3024 & 6.9079 & 113.836 & 301.667 & 0.02053 & 0.002 & 0.15 & 0.03941 \\
\hline 3025 & 6.9076 & 113.744 & 301.853 & 0.02055 & 0.002 & 0.24 & 0.04484 \\
\hline 3026 & 6.9076 & 113.652 & 302.061 & 0.02051 & 0.001 & 0.00 & 0.05062 \\
\hline 3027 & 6.9077 & 113.560 & 302.270 & 0.02055 & 0.001 & 0.14 & 0.05676 \\
\hline 3028 & 6.9078 & 113.460 & 302.495 & 0.02056 & 0.001 & 0.13 & 0.06324 \\
\hline 3029 & 6.9078 & 113.348 & 302.744 & 0.02061 & 0.001 & 0.30 & 0.07007 \\
\hline 3030 & 6.9078 & 113.237 & 302.994 & 0.02062 & 0.001 & 0.30 & 0.07726 \\
\hline 3043 & 6.2650 & 103.038 & 301.530 & 0.02027 & 0.003 & 0.28 & 0.03433 \\
\hline 3044 & 6.2650 & 102.962 & 301.714 & 0.02029 & 0.002 & 0.35 & 0.03942 \\
\hline 3045 & 6.2651 & 102.886 & 301.910 & 0.02026 & 0.002 & 0.16 & 0.04485 \\
\hline 3046 & 6.2651 & 102.806 & 302.110 & 0.02027 & 0.001 & 0.15 & 0.05063 \\
\hline 3047 & 6.2651 & 102.714 & 302.333 & 0.02024 & 0.001 & -0.06 & 0.05676 \\
\hline 3048 & 6.2652 & 102.622 & 302.566 & 0.02026 & 0.001 & -0.01 & 0.06325 \\
\hline 3049 & 6.2652 & 102.527 & 302.805 & 0.02029 & 0.001 & 0.12 & 0.07008 \\
\hline 3050 & 6.2652 & 102.423 & 303.069 & 0.02032 & 0.001 & 0.21 & 0.07727 \\
\hline 3062 & 5.5849 & 91.645 & 301.383 & 0.01991 & 0.003 & -0.02 & 0.02961 \\
\hline 3063 & 5.5849 & 91.581 & 301.557 & 0.01990 & 0.002 & -0.11 & 0.03434 \\
\hline 3064 & 5.5851 & 91.521 & 301.737 & 0.01994 & 0.002 & 0.03 & 0.03942 \\
\hline 3065 & 5.5849 & 91.445 & 301.942 & 0.01987 & 0.002 & -0.39 & 0.04485 \\
\hline 3066 & 5.5851 & 91.377 & 302.150 & 0.01997 & 0.002 & 0.09 & 0.05063 \\
\hline 3067 & 5.5850 & 91.293 & 302.377 & 0.01998 & 0.001 & 0.09 & 0.05676 \\
\hline 3068 & 5.5848 & 91.201 & 302.628 & 0.02003 & 0.002 & 0.30 & 0.06325 \\
\hline 3069 & 5.5848 & 91.121 & 302.859 & 0.02001 & 0.001 & 0.10 & 0.07008 \\
\hline 3070 & 5.5849 & 91.034 & 303.121 & 0.02001 & 0.001 & 0.04 & 0.07727 \\
\hline 3082 & 4.9512 & 81.011 & 301.407 & 0.01965 & 0.003 & -0.03 & 0.02961 \\
\hline 3083 & 4.9514 & 80.959 & 301.589 & 0.01966 & 0.002 & -0.04 & 0.03434 \\
\hline 3084 & 4.9514 & 80.899 & 301.776 & 0.01956 & 0.002 & -0.61 & 0.03942 \\
\hline 3085 & 4.9516 & 80.839 & 301.980 & 0.01978 & 0.002 & 0.50 & 0.04485 \\
\hline 3086 & 4.9517 & 80.775 & 302.198 & 0.01976 & 0.001 & 0.31 & 0.05063 \\
\hline 3087 & 4.9517 & 80.707 & 302.427 & 0.01968 & 0.001 & -0.15 & 0.05677 \\
\hline 3088 & 4.9518 & 80.631 & 302.669 & 0.01962 & 0.001 & -0.48 & 0.06325 \\
\hline 3089 & 4.9519 & 80.555 & 302.926 & 0.01970 & 0.001 & -0.16 & 0.07008 \\
\hline 3090 & 4.9519 & 80.475 & 303.193 & 0.01968 & 0.001 & -0.32 & 0.07727 \\
\hline 3102 & 4.2568 & 69.414 & 301.448 & 0.01914 & 0.003 & -1.27 & 0.02961 \\
\hline 3103 & 4.2569 & 69.370 & 301.624 & 0.01928 & 0.002 & -0.61 & 0.03434 \\
\hline 3104 & 4.2569 & 69.318 & 301.821 & 0.01935 & 0.002 & -0.26 & 0.03942 \\
\hline 3105 & 4.2570 & 69.266 & 302.025 & 0.01936 & 0.002 & -0.28 & 0.04485 \\
\hline 3106 & 4.2570 & 69.206 & 302.258 & 0.01938 & 0.001 & -0.24 & 0.05063 \\
\hline 3107 & 4.2572 & 69.150 & 302.495 & 0.01934 & 0.001 & -0.49 & 0.05677 \\
\hline 3108 & 4.2569 & 69.082 & 302.739 & 0.01944 & 0.001 & -0.04 & 0.06325 \\
\hline 3109 & 4.2570 & 69.014 & 303.009 & 0.01942 & 0.001 & -0.23 & 0.07009 \\
\hline 3110 & 4.2572 & 68.946 & 303.284 & 0.01941 & 0.001 & -0.32 & 0.07728 \\
\hline 3123 & 3.5548 & 57.757 & 301.495 & 0.01883 & 0.003 & -1.56 & 0.02961 \\
\hline 3124 & 3.5545 & 57.713 & 301.678 & 0.01905 & 0.002 & -0.42 & 0.03434 \\
\hline 3125 & 3.5546 & 57.669 & 301.892 & 0.01902 & 0.002 & -0.64 & 0.03942 \\
\hline
\end{tabular}


Table 2. Thermal conductivity of argon, transient method-Continued

\begin{tabular}{|c|c|c|c|c|c|c|c|}
\hline $\begin{array}{c}\text { Point } \\
\text { no. }\end{array}$ & $\begin{array}{c}p \\
(\mathrm{MPa})\end{array}$ & $\begin{array}{c}\rho \\
\left(\mathrm{kg} \mathrm{m}^{-3}\right)\end{array}$ & $\begin{array}{l}T_{\exp } \\
(\mathrm{K})\end{array}$ & $\begin{array}{c}\lambda_{\exp } \\
\left(\mathrm{W} \mathrm{m}^{-1} \mathrm{~K}^{-1}\right)\end{array}$ & STAT & $\begin{array}{l}\text { Relative } \\
\text { dev. }(\%)\end{array}$ & $\begin{array}{c}q \\
\left(\mathrm{~W} \mathrm{~m} \mathrm{~m}^{-1}\right)\end{array}$ \\
\hline \multicolumn{8}{|c|}{ Nominal temperature $300 \mathrm{~K}$} \\
\hline 3126 & 3.5546 & 57.625 & 302.108 & 0.01906 & 0.002 & -0.46 & 0.04485 \\
\hline 3127 & 3.5546 & 57.577 & 302.335 & 0.01907 & 0.001 & -0.49 & 0.05064 \\
\hline 3128 & 3.5547 & 57.529 & 302.581 & 0.01906 & 0.001 & -0.60 & 0.05677 \\
\hline 3129 & 3.5547 & 57.473 & 302.836 & 0.01911 & 0.001 & -0.39 & 0.06326 \\
\hline 3130 & 3.5545 & 57.413 & 303.112 & 0.01912 & 0.001 & -0.39 & 0.07009 \\
\hline 4003 & 2.8395 & 45.944 & 301.638 & 0.01883 & 0.003 & -0.18 & 0.02960 \\
\hline 4004 & 2.8397 & 45.916 & 301.831 & 0.01873 & 0.002 & -0.78 & 0.03433 \\
\hline 4005 & 2.8397 & 45.880 & 302.047 & 0.01881 & 0.002 & -0.38 & 0.03941 \\
\hline 4006 & 2.8397 & 45.844 & 302.264 & 0.01884 & 0.002 & -0.28 & 0.04484 \\
\hline 4007 & 2.8397 & 45.804 & 302.508 & 0.01882 & 0.001 & -0.44 & 0.05063 \\
\hline 4008 & 2.8397 & 45.764 & 302.765 & 0.01879 & 0.001 & -0.70 & 0.05676 \\
\hline 4009 & 2.8398 & 45.720 & 303.036 & 0.01883 & 0.001 & -0.53 & 0.06324 \\
\hline 4010 & 2.8397 & 45.673 & 303.324 & 0.01883 & 0.001 & -0.63 & 0.07007 \\
\hline 4023 & 2.1132 & 34.056 & 301.681 & 0.01850 & 0.003 & -0.60 & 0.02962 \\
\hline 4024 & 2.1132 & 34.032 & 301.896 & 0.01844 & 0.003 & -0.97 & 0.03435 \\
\hline 4025 & 2.1131 & 34.004 & 302.124 & 0.01821 & 0.002 & -2.32 & 0.03943 \\
\hline 4026 & 2.1130 & 33.976 & 302.346 & 0.01846 & 0.002 & -0.95 & 0.04487 \\
\hline 4027 & 2.1131 & 33.948 & 302.592 & 0.01844 & 0.001 & -1.14 & 0.05065 \\
\hline 4028 & 2.1132 & 33.916 & 302.857 & 0.01853 & 0.001 & -0.74 & 0.05679 \\
\hline 4029 & 2.1131 & 33.880 & 303.141 & 0.01847 & 0.001 & -1.15 & 0.06328 \\
\hline 4030 & 2.1132 & 33.848 & 303.444 & 0.01847 & 0.001 & -1.21 & 0.07011 \\
\hline 4045 & 1.3696 & 21.963 & 301.960 & 0.01808 & 0.002 & -1.58 & 0.03435 \\
\hline 4046 & 1.3696 & 21.947 & 302.195 & 0.01807 & 0.002 & -1.73 & 0.03943 \\
\hline 4047 & 1.3696 & 21.927 & 302.446 & 0.01815 & 0.002 & -1.36 & 0.04487 \\
\hline 4048 & 1.3697 & 21.907 & 302.709 & 0.01820 & 0.001 & -1.11 & 0.05065 \\
\hline 4049 & 1.3697 & 21.888 & 302.995 & 0.01817 & 0.001 & -1.39 & 0.05679 \\
\hline 4050 & 1.3697 & 21.864 & 303.298 & 0.01814 & 0.001 & -1.64 & 0.06327 \\
\hline 4084 & 0.7010 & 11.197 & 301.997 & 0.01788 & 0.003 & -1.53 & 0.03435 \\
\hline 4085 & 0.7012 & 11.189 & 302.256 & 0.01803 & 0.003 & -0.75 & 0.03943 \\
\hline 4086 & 0.7010 & 11.177 & 302.533 & 0.01813 & 0.003 & -0.26 & 0.04487 \\
\hline 4087 & 0.7010 & 11.165 & 302.796 & 0.01788 & 0.002 & -1.79 & 0.05065 \\
\hline 4088 & 0.7011 & 11.157 & 303.109 & 0.01793 & 0.002 & -1.56 & 0.05679 \\
\hline 4089 & 0.7011 & 11.145 & 303.417 & 0.01777 & 0.002 & -2.58 & 0.06327 \\
\hline 4090 & 0.7011 & 11.134 & 303.762 & 0.01785 & 0.003 & -2.21 & 0.07011 \\
\hline 4107 & 0.3782 & 6.024 & 302.352 & 0.01763 & 0.003 & -2.49 & 0.03943 \\
\hline 4108 & 0.3784 & 6.020 & 302.636 & 0.01768 & 0.002 & -2.30 & 0.04487 \\
\hline 4109 & 0.3784 & 6.016 & 302.930 & 0.01768 & 0.002 & -2.36 & 0.05065 \\
\hline 4110 & 0.3783 & 6.008 & 303.254 & 0.01765 & 0.002 & -2.62 & 0.05679 \\
\hline 4127 & 0.1684 & 2.681 & 302.206 & 0.01786 & 0.003 & -0.75 & 0.03435 \\
\hline 4128 & 0.1686 & 2.681 & 302.500 & 0.01792 & 0.003 & -0.50 & 0.03943 \\
\hline 4129 & 0.1686 & 2.677 & 302.817 & 0.01794 & 0.003 & -0.51 & 0.04487 \\
\hline 4130 & 0.1686 & 2.673 & 303.142 & 0.01806 & 0.003 & 0.10 & 0.05065 \\
\hline
\end{tabular}

Nominal temperature $320 \mathrm{~K}$

\begin{tabular}{|c|c|c|c|c|c|c|c|}
\hline 5001 & 8.3272 & 127.678 & 321.430 & 0.02186 & 0.002 & -0.49 & 0.04215 \\
\hline 5002 & 8.3273 & 127.630 & 321.533 & 0.02193 & 0.002 & -0.20 & 0.04501 \\
\hline 5003 & 8.3273 & 127.590 & 321.617 & 0.02197 & 0.002 & -0.02 & 0.04796 \\
\hline 5004 & 8.3273 & 127.542 & 321.720 & 0.02189 & 0.002 & -0.41 & 0.05100 \\
\hline 5005 & 8.3275 & 127.494 & 321.822 & 0.02188 & 0.001 & -0.47 & 0.05414 \\
\hline 5006 & 8.3273 & 127.442 & 321.927 & 0.02198 & 0.001 & -0.05 & 0.05737 \\
\hline 5007 & 8.3275 & 127.394 & 322.036 & 0.02188 & 0.001 & -0.49 & 0.06069 \\
\hline 5008 & 8.3277 & 127.346 & 322.145 & 0.02193 & 0.001 & -0.31 & 0.06411 \\
\hline 5009 & 8.3275 & 127.286 & 322.262 & 0.02195 & 0.001 & -0.22 & 0.06762 \\
\hline 5010 & 8.3275 & 127.234 & 322.379 & 0.02190 & 0.001 & -0.46 & 0.07123 \\
\hline 5021 & 7.5805 & 116.005 & 321.557 & 0.02162 & 0.002 & -0.16 & 0.04501 \\
\hline
\end{tabular}


Table 2. Thermal conductivity of argon, transient method-Continued

\begin{tabular}{|c|c|c|c|c|c|c|c|}
\hline $\begin{array}{l}\text { Point } \\
\text { no. }\end{array}$ & $\begin{array}{c}p \\
(\mathrm{MPa})\end{array}$ & $\begin{array}{c}\rho \\
\left(\mathrm{kg} \mathrm{m}^{-3}\right)\end{array}$ & $\begin{array}{l}T_{\exp } \\
(\mathrm{K})\end{array}$ & $\begin{array}{c}\lambda_{\exp } \\
\left(\mathrm{W} \mathrm{m}^{-1} \mathrm{~K}^{-1}\right)\end{array}$ & STAT & $\begin{array}{l}\text { Relative } \\
\text { dev. }(\%)\end{array}$ & $\begin{array}{c}q \\
\left(\mathrm{~W} \mathrm{~m}^{-1}\right)\end{array}$ \\
\hline \multicolumn{8}{|c|}{ Nominal temperature $320 \mathrm{~K}$} \\
\hline 5022 & 7.5807 & 115.925 & 321.760 & 0.02164 & 0.002 & -0.07 & 0.05100 \\
\hline 5023 & 7.5808 & 115.837 & 321.967 & 0.02157 & 0.001 & -0.45 & 0.05737 \\
\hline 5024 & 7.5808 & 115.745 & 322.187 & 0.02157 & 0.001 & -0.53 & 0.06411 \\
\hline 5025 & 7.5808 & 115.645 & 322.424 & 0.02157 & 0.001 & -0.54 & 0.07123 \\
\hline 5026 & 7.5809 & 115.542 & 322.675 & 0.02155 & 0.001 & -0.71 & 0.07871 \\
\hline 5027 & 7.5812 & 115.438 & 322.931 & 0.02161 & 0.001 & -0.49 & 0.08658 \\
\hline 5028 & 7.5811 & 115.322 & 323.204 & 0.02160 & 0.001 & -0.55 & 0.09482 \\
\hline 5029 & 7.5812 & 115.202 & 323.490 & 0.02163 & 0.001 & -0.50 & 0.10343 \\
\hline 5030 & 7.5812 & 115.078 & 323.792 & 0.02162 & 0.001 & -0.57 & 0.11241 \\
\hline 5041 & 6.8721 & 104.995 & 321.578 & 0.02130 & 0.002 & -0.26 & 0.04501 \\
\hline 5042 & 6.8720 & 104.915 & 321.785 & 0.02129 & 0.001 & -0.35 & 0.05100 \\
\hline 5043 & 6.8720 & 104.836 & 321.999 & 0.02139 & 0.001 & 0.07 & 0.05737 \\
\hline 5044 & 6.8721 & 104.744 & 322.246 & 0.02134 & 0.001 & -0.24 & 0.06411 \\
\hline 5045 & 6.8721 & 104.652 & 322.484 & 0.02135 & 0.001 & -0.25 & 0.07122 \\
\hline 5046 & 6.8721 & 104.560 & 322.735 & 0.02128 & 0.001 & -0.63 & 0.07871 \\
\hline 5047 & 6.8721 & 104.460 & 323.002 & 0.02136 & 0.001 & -0.29 & 0.08658 \\
\hline 5048 & 6.8721 & 104.356 & 323.279 & 0.02125 & 0.001 & -0.85 & 0.09481 \\
\hline 5049 & 6.8722 & 104.248 & 323.571 & 0.02146 & 0.001 & 0.06 & 0.10343 \\
\hline 5050 & 6.8721 & 104.128 & 323.884 & 0.02143 & 0.001 & -0.16 & 0.11241 \\
\hline 5061 & 6.1871 & 94.369 & 321.598 & 0.02100 & 0.002 & -0.37 & 0.04500 \\
\hline 5062 & 6.1870 & 94.297 & 321.809 & 0.02099 & 0.002 & -0.50 & 0.05099 \\
\hline 5064 & 6.1869 & 94.133 & 322.288 & 0.02100 & 0.001 & -0.56 & 0.06410 \\
\hline 5065 & 6.1869 & 94.054 & 322.526 & 0.02097 & 0.001 & -0.72 & 0.07122 \\
\hline 5066 & 6.1868 & 93.970 & 322.773 & 0.02104 & 0.001 & -0.43 & 0.07871 \\
\hline 5067 & 6.1868 & 93.882 & 323.047 & 0.02106 & 0.001 & -0.42 & 0.08657 \\
\hline 5068 & 6.1867 & 93.782 & 323.335 & 0.02100 & 0.001 & -0.78 & 0.09481 \\
\hline 5069 & 6.1866 & 93.682 & 323.629 & 0.02103 & 0.001 & -0.70 & 0.10342 \\
\hline 5070 & 6.1866 & 93.578 & 323.942 & 0.02106 & 0.001 & -0.62 & 0.11241 \\
\hline 5082 & 5.5060 & 83.859 & 321.512 & 0.02057 & 0.002 & -1.18 & 0.04215 \\
\hline 5083 & 5.5060 & 83.803 & 321.705 & 0.02073 & 0.002 & -0.43 & 0.04795 \\
\hline 5084 & 5.5061 & 83.735 & 321.934 & 0.02077 & 0.001 & -0.28 & 0.05414 \\
\hline 5085 & 5.5061 & 83.667 & 322.164 & 0.02073 & 0.001 & -0.52 & 0.06069 \\
\hline 5086 & 5.5061 & 83.587 & 322.430 & 0.02068 & 0.002 & -0.82 & 0.06762 \\
\hline 5087 & 5.5061 & 83.515 & 322.685 & 0.02074 & 0.001 & -0.58 & 0.07492 \\
\hline 5088 & 5.5061 & 83.435 & 322.959 & 0.02077 & 0.001 & -0.51 & 0.08260 \\
\hline 5089 & 5.5061 & 83.352 & 323.250 & 0.02064 & 0.001 & -1.21 & 0.09065 \\
\hline 5090 & 5.5061 & 83.268 & 323.532 & 0.02071 & 0.001 & -0.93 & 0.09908 \\
\hline 5103 & 4.8431 & 73.672 & 321.333 & 0.02043 & 0.003 & -0.58 & 0.03672 \\
\hline 5104 & 4.8431 & 73.624 & 321.518 & 0.02042 & 0.002 & -0.64 & 0.04215 \\
\hline 5105 & 4.8432 & 73.572 & 321.728 & 0.02048 & 0.002 & -0.40 & 0.04796 \\
\hline 5106 & 4.8431 & 73.512 & 321.960 & 0.02048 & 0.002 & -0.44 & 0.05414 \\
\hline 5107 & 4.8432 & 73.452 & 322.198 & 0.02048 & 0.001 & -0.52 & 0.06069 \\
\hline 5108 & 4.8431 & 73.388 & 322.449 & 0.02048 & 0.001 & -0.59 & 0.06762 \\
\hline 5109 & 4.8431 & 73.321 & 322.712 & 0.02046 & 0.001 & -0.74 & 0.07492 \\
\hline 5110 & 4.8431 & 73.249 & 323.000 & 0.02049 & 0.001 & -0.66 & 0.08260 \\
\hline 5122 & 4.1373 & 62.842 & 321.147 & 0.02004 & 0.003 & -1.14 & 0.03166 \\
\hline 5123 & 4.1373 & 62.798 & 321.339 & 0.02018 & 0.003 & -0.50 & 0.03672 \\
\hline 5124 & 4.1374 & 62.758 & 321.541 & 0.02023 & 0.002 & -0.31 & 0.04215 \\
\hline 5125 & 4.1374 & 62.706 & 321.776 & 0.02013 & 0.002 & -0.84 & 0.04796 \\
\hline 5126 & 4.1375 & 62.658 & 322.005 & 0.02021 & 0.002 & -0.53 & 0.05414 \\
\hline 5127 & 4.1375 & 62.603 & 322.266 & 0.02012 & 0.002 & -1.02 & 0.06069 \\
\hline 5128 & 4.1375 & 62.547 & 322.531 & 0.02031 & 0.001 & -0.15 & 0.06762 \\
\hline 5129 & 4.1375 & 62.491 & 322.791 & 0.02023 & 0.001 & -0.57 & 0.07492 \\
\hline 5130 & 4.1375 & 62.431 & 323.081 & 0.02020 & 0.001 & -0.80 & 0.08260 \\
\hline 5143 & 3.4551 & 52.320 & 321.388 & 0.01992 & 0.003 & -0.61 & 0.03672 \\
\hline 5145 & 3.4549 & 52.240 & 321.841 & 0.01989 & 0.002 & -0.83 & 0.04795 \\
\hline
\end{tabular}


Table 2. Thermal conductivity of argon, transient method-Continued

\begin{tabular}{|c|c|c|c|c|c|c|c|}
\hline $\begin{array}{l}\text { Point } \\
\text { no. }\end{array}$ & $\begin{array}{c}p \\
(\mathrm{MPa})\end{array}$ & $\begin{array}{c}\rho \\
\left(\mathrm{kg} \mathrm{m}^{-3}\right)\end{array}$ & $\begin{array}{l}T_{\exp } \\
(\mathrm{K})\end{array}$ & $\begin{array}{c}\lambda_{\exp } \\
\left(\mathrm{W} \mathrm{m}^{-1} \mathrm{~K}^{-1}\right)\end{array}$ & STAT & $\begin{array}{l}\text { Relative } \\
\text { dev. (\%) }\end{array}$ & $\begin{array}{c}q \\
\left(\mathrm{~W} \mathrm{~m}^{-1}\right)\end{array}$ \\
\hline \multicolumn{8}{|c|}{ Nominal temperature $320 \mathrm{~K}$} \\
\hline 5146 & 3.4550 & 52.200 & 322.077 & 0.02004 & 0.002 & -0.12 & 0.05413 \\
\hline 5147 & 3.4550 & 52.156 & 322.325 & 0.01990 & 0.001 & -0.91 & 0.06069 \\
\hline 5148 & 3.4546 & 52.104 & 322.584 & 0.01991 & 0.001 & -0.90 & 0.06762 \\
\hline 5149 & 3.4548 & 52.052 & 322.889 & 0.01994 & 0.001 & -0.86 & 0.07492 \\
\hline 5150 & 3.4549 & 52.000 & 323.191 & 0.01995 & 0.001 & -0.86 & 0.08259 \\
\hline 5163 & 2.7632 & 41.770 & 321.224 & 0.01969 & 0.003 & -0.49 & 0.03165 \\
\hline 5164 & 2.7631 & 41.738 & 321.429 & 0.01961 & 0.003 & -0.93 & 0.03671 \\
\hline 5165 & 2.7632 & 41.706 & 321.678 & 0.01973 & 0.002 & -0.36 & 0.04214 \\
\hline 5166 & 2.7633 & 41.678 & 321.896 & 0.01964 & 0.002 & -0.92 & 0.04795 \\
\hline 5167 & 2.7633 & 41.642 & 322.159 & 0.01966 & 0.002 & -0.84 & 0.05413 \\
\hline 5168 & 2.7632 & 41.602 & 322.422 & 0.01960 & 0.002 & -1.23 & 0.06068 \\
\hline 5169 & 2.7633 & 41.566 & 322.688 & 0.01964 & 0.001 & -1.11 & 0.06761 \\
\hline 5170 & 2.7633 & 41.526 & 322.985 & 0.01966 & 0.001 & -1.08 & 0.07491 \\
\hline 5193 & 2.0865 & 31.455 & 321.287 & 0.01916 & 0.003 & -2.06 & 0.03165 \\
\hline 5194 & 2.0865 & 31.435 & 321.494 & 0.01932 & 0.003 & -1.28 & 0.03671 \\
\hline 5195 & 2.0864 & 31.411 & 321.726 & 0.01929 & 0.002 & -1.50 & 0.04215 \\
\hline 5196 & 2.0866 & 31.387 & 321.970 & 0.01932 & 0.002 & -1.41 & 0.04795 \\
\hline 5197 & 2.0865 & 31.359 & 322.228 & 0.01934 & 0.002 & -1.36 & 0.05413 \\
\hline 5198 & 2.0865 & 31.331 & 322.499 & 0.01933 & 0.001 & -1.48 & 0.06068 \\
\hline 5199 & 2.0863 & 31.299 & 322.791 & 0.01935 & 0.001 & -1.45 & 0.06761 \\
\hline 5200 & 2.0863 & 31.267 & 323.096 & 0.01938 & 0.001 & -1.34 & 0.07491 \\
\hline 5214 & 1.3800 & 20.729 & 321.581 & 0.01897 & 0.003 & -1.91 & 0.03671 \\
\hline 5215 & 1.3798 & 20.713 & 321.822 & 0.01902 & 0.002 & -1.74 & 0.04214 \\
\hline 5216 & 1.3798 & 20.697 & 322.075 & 0.01898 & 0.003 & -2.03 & 0.04795 \\
\hline 5217 & 1.3798 & 20.677 & 322.346 & 0.01902 & 0.002 & -1.85 & 0.05412 \\
\hline 5218 & 1.3800 & 20.661 & 322.636 & 0.01895 & 0.001 & -2.33 & 0.06068 \\
\hline 5219 & 1.3800 & 20.641 & 322.948 & 0.01915 & 0.002 & -1.34 & 0.06760 \\
\hline 5220 & 1.3800 & 20.617 & 323.267 & 0.01895 & 0.001 & -2.45 & 0.07490 \\
\hline 5235 & 0.6849 & 10.251 & 321.919 & 0.01867 & 0.003 & -2.49 & 0.04214 \\
\hline 5236 & 0.6849 & 10.239 & 322.206 & 0.01894 & 0.003 & -1.06 & 0.04794 \\
\hline 5237 & 0.6849 & 10.231 & 322.494 & 0.01870 & 0.002 & -2.48 & 0.05412 \\
\hline 5239 & 0.6849 & 10.211 & 323.147 & 0.01891 & 0.002 & -1.47 & 0.06760 \\
\hline 5240 & 0.6849 & 10.203 & 323.465 & 0.01880 & 0.001 & -2.18 & 0.07490 \\
\hline 5259 & 0.3678 & 5.485 & 322.625 & 0.01864 & 0.002 & -2.27 & 0.05412 \\
\hline 5260 & 0.3679 & 5.481 & 322.970 & 0.01865 & 0.002 & -2.31 & 0.06067 \\
\hline 5277 & 0.1732 & 2.581 & 322.807 & 0.01881 & 0.002 & -1.09 & 0.05412 \\
\hline 5278 & 0.1734 & 2.577 & 323.722 & 0.01885 & 0.002 & -1.09 & 0.07120 \\
\hline 5279 & 0.1733 & 2.565 & 324.762 & 0.01893 & 0.002 & -0.96 & 0.09062 \\
\hline
\end{tabular}

\begin{tabular}{|c|c|c|c|c|c|c|c|}
\hline \multicolumn{8}{|c|}{ Nominal temperature $340 \mathrm{~K}$} \\
\hline 6001 & 8.1063 & 112.607 & 342.889 & 0.02259 & 0.001 & -0.74 & 0.12914 \\
\hline 6002 & 8.1064 & 112.786 & 342.417 & 0.02257 & 0.001 & -0.77 & 0.11441 \\
\hline 6003 & 8.1065 & 112.954 & 341.979 & 0.02252 & 0.001 & -0.89 & 0.10058 \\
\hline 6004 & 8.1066 & 113.118 & 341.557 & 0.02247 & 0.001 & -1.08 & 0.08763 \\
\hline 6005 & 8.1067 & 113.267 & 341.170 & 0.02253 & 0.001 & -0.72 & 0.07556 \\
\hline 6006 & 8.1068 & 113.403 & 340.814 & 0.02246 & 0.001 & -0.95 & 0.06439 \\
\hline 6018 & 7.5842 & 105.344 & 342.791 & 0.02238 & 0.001 & -0.76 & 0.12412 \\
\hline 6019 & 7.5843 & 105.453 & 342.487 & 0.02233 & 0.001 & -0.94 & 0.11439 \\
\hline 6020 & 7.5845 & 105.566 & 342.175 & 0.02236 & 0.001 & -0.76 & 0.10507 \\
\hline 6021 & 7.5846 & 105.664 & 341.892 & 0.02236 & 0.001 & -0.70 & 0.09614 \\
\hline 6022 & 7.5845 & 105.762 & 341.615 & 0.02228 & 0.001 & -0.98 & 0.08761 \\
\hline 6023 & 7.5848 & 105.859 & 341.349 & 0.02232 & 0.001 & -0.76 & 0.07948 \\
\hline 6024 & 7.5848 & 105.949 & 341.101 & 0.02231 & 0.001 & -0.79 & 0.07173 \\
\hline 6036 & 6.9060 & 95.828 & 342.896 & 0.02210 & 0.001 & -0.89 & 0.12412 \\
\hline 6037 & 6.9061 & 95.933 & 342.567 & 0.02208 & 0.001 & -0.89 & 0.11440 \\
\hline
\end{tabular}


Table 2. Thermal conductivity of argon, transient method-Continued

\begin{tabular}{|c|c|c|c|c|c|c|c|}
\hline $\begin{array}{l}\text { Point } \\
\text { no. }\end{array}$ & $\begin{array}{c}p \\
(\mathrm{MPa})\end{array}$ & $\begin{array}{c}\rho \\
\left(\mathrm{kg} \mathrm{m}^{-3}\right)\end{array}$ & $\begin{array}{l}T_{\exp } \\
(\mathrm{K})\end{array}$ & $\begin{array}{c}\lambda_{\exp } \\
\left(\mathrm{W} \mathrm{m}^{-1} \mathrm{~K}^{-1}\right)\end{array}$ & STAT & $\begin{array}{l}\text { Relative } \\
\text { dev. }(\%)\end{array}$ & $\begin{array}{c}q \\
\left(\mathrm{~W} \mathrm{~m}^{-1}\right)\end{array}$ \\
\hline \multicolumn{8}{|c|}{ Nominal temperature $340 \mathrm{~K}$} \\
\hline 6038 & 6.9062 & 96.027 & 342.270 & 0.02199 & 0.002 & -1.27 & 0.10508 \\
\hline 6039 & 6.9065 & 96.128 & 341.971 & 0.02205 & 0.001 & -0.95 & 0.09615 \\
\hline 6040 & 6.9062 & 96.214 & 341.683 & 0.02212 & 0.001 & -0.55 & 0.08761 \\
\hline 6041 & 6.9064 & 96.304 & 341.419 & 0.02200 & 0.001 & -1.05 & 0.07948 \\
\hline 6042 & 6.9065 & 96.386 & 341.167 & 0.02198 & 0.002 & -1.07 & 0.07173 \\
\hline 6054 & 6.2153 & 86.152 & 342.983 & 0.02187 & 0.001 & -0.77 & 0.12412 \\
\hline 6055 & 6.2153 & 86.241 & 342.663 & 0.02188 & 0.001 & -0.66 & 0.11440 \\
\hline 6056 & 6.2154 & 86.327 & 342.362 & 0.02186 & 0.001 & -0.66 & 0.10508 \\
\hline 6057 & 6.2155 & 86.413 & 342.061 & 0.02173 & 0.002 & -1.20 & 0.09615 \\
\hline 6058 & 6.2156 & 86.499 & 341.768 & 0.02184 & 0.001 & -0.67 & 0.08762 \\
\hline 6059 & 6.2155 & 86.573 & 341.498 & 0.02182 & 0.001 & -0.68 & 0.07948 \\
\hline 6060 & 6.2156 & 86.651 & 341.226 & 0.02185 & 0.001 & -0.49 & 0.07174 \\
\hline 6072 & 5.5214 & 76.433 & 343.103 & 0.02158 & 0.001 & -0.93 & 0.12412 \\
\hline 6073 & 5.5215 & 76.518 & 342.765 & 0.02155 & 0.001 & -1.01 & 0.11440 \\
\hline 6074 & 5.5216 & 76.597 & 342.443 & 0.02158 & 0.001 & -0.82 & 0.10507 \\
\hline 6075 & 5.5215 & 76.675 & 342.137 & 0.02153 & 0.001 & -0.95 & 0.09615 \\
\hline 6076 & 5.5216 & 76.749 & 341.833 & 0.02153 & 0.001 & -0.92 & 0.08761 \\
\hline 6077 & 5.5215 & 76.819 & 341.552 & 0.02151 & 0.001 & -0.96 & 0.07948 \\
\hline 6078 & 5.5216 & 76.886 & 341.288 & 0.02155 & 0.001 & -0.72 & 0.07173 \\
\hline 6090 & 4.8340 & 66.823 & 343.216 & 0.02135 & 0.001 & -0.90 & 0.12412 \\
\hline 6091 & 4.8341 & 66.901 & 342.864 & 0.02134 & 0.001 & -0.85 & 0.11440 \\
\hline 6092 & 4.8341 & 66.971 & 342.531 & 0.02128 & 0.001 & -1.06 & 0.10508 \\
\hline 6093 & 4.8340 & 67.038 & 342.223 & 0.02124 & 0.001 & -1.19 & 0.09614 \\
\hline 6094 & 4.8341 & 67.104 & 341.915 & 0.02132 & 0.001 & -0.76 & 0.08762 \\
\hline 6095 & 4.8342 & 67.170 & 341.622 & 0.02125 & 0.001 & -1.02 & 0.07948 \\
\hline 6096 & 4.8341 & 67.225 & 341.353 & 0.02121 & 0.001 & -1.14 & 0.07174 \\
\hline 6108 & 4.1410 & 57.158 & 343.345 & 0.02106 & 0.001 & -1.12 & 0.12414 \\
\hline 6109 & 4.1411 & 57.221 & 342.987 & 0.02107 & 0.001 & -0.98 & 0.11441 \\
\hline 6110 & 4.1409 & 57.280 & 342.651 & 0.02106 & 0.001 & -0.98 & 0.10508 \\
\hline 6112 & 4.1408 & 57.397 & 342.005 & 0.02100 & 0.001 & -1.12 & 0.08762 \\
\hline 6113 & 4.1410 & 57.455 & 341.706 & 0.02095 & 0.001 & -1.31 & 0.07949 \\
\hline 6114 & 4.1411 & 57.506 & 341.425 & 0.02105 & 0.001 & -0.77 & 0.07174 \\
\hline 6126 & 3.4501 & 47.568 & 343.297 & 0.02085 & 0.001 & -0.97 & 0.11921 \\
\hline 6127 & 3.4501 & 47.619 & 342.946 & 0.02077 & 0.001 & -1.30 & 0.10969 \\
\hline 6128 & 3.4499 & 47.670 & 342.599 & 0.02071 & 0.001 & -1.49 & 0.10057 \\
\hline 6130 & 3.4495 & 47.760 & 341.955 & 0.02080 & 0.001 & -0.94 & 0.08351 \\
\hline 6131 & 3.4496 & 47.806 & 341.653 & 0.02077 & 0.001 & -0.99 & 0.07556 \\
\hline 6132 & 3.4496 & 47.849 & 341.371 & 0.02074 & 0.001 & -1.10 & 0.06802 \\
\hline 6144 & 3.4481 & 47.537 & 343.306 & 0.02081 & 0.001 & -1.18 & 0.11923 \\
\hline 6145 & 3.4481 & 47.592 & 342.944 & 0.02078 & 0.001 & -1.25 & 0.10970 \\
\hline 6146 & 3.4483 & 47.646 & 342.606 & 0.02075 & 0.001 & -1.30 & 0.10058 \\
\hline 6147 & 3.4481 & 47.693 & 342.273 & 0.02072 & 0.001 & -1.37 & 0.09184 \\
\hline 6148 & 3.4481 & 47.740 & 341.956 & 0.02078 & 0.001 & -1.03 & 0.08351 \\
\hline 6149 & 3.4481 & 47.783 & 341.663 & 0.02074 & 0.001 & -1.18 & 0.07556 \\
\hline 6150 & 3.4481 & 47.826 & 341.373 & 0.02074 & 0.001 & -1.08 & 0.06802 \\
\hline 6162 & 2.7654 & 38.056 & 343.459 & 0.02057 & 0.001 & -1.27 & 0.11920 \\
\hline 6163 & 2.7655 & 38.103 & 343.089 & 0.02057 & 0.001 & -1.17 & 0.10968 \\
\hline 6164 & 2.7655 & 38.142 & 342.738 & 0.02049 & 0.001 & -1.52 & 0.10056 \\
\hline 6165 & 2.7656 & 38.189 & 342.377 & 0.02049 & 0.001 & -1.41 & 0.09184 \\
\hline 6166 & 2.7655 & 38.224 & 342.061 & 0.02055 & 0.001 & -1.03 & 0.08350 \\
\hline 6167 & 2.7655 & 38.259 & 341.746 & 0.02050 & 0.001 & -1.25 & 0.07557 \\
\hline 6168 & 2.7655 & 38.294 & 341.445 & 0.02050 & 0.001 & -1.17 & 0.06802 \\
\hline 6191 & 2.0713 & 28.462 & 343.464 & 0.02017 & 0.001 & -2.15 & 0.11439 \\
\hline 6192 & 2.0713 & 28.497 & 343.073 & 0.02025 & 0.001 & -1.65 & 0.10508 \\
\hline 6193 & 2.0713 & 28.528 & 342.708 & 0.02021 & 0.001 & -1.79 & 0.09615 \\
\hline 6194 & 2.0714 & 28.560 & 342.363 & 0.02021 & 0.001 & -1.67 & 0.08762 \\
\hline
\end{tabular}


Table 2. Thermal conductivity of argon, transient method-Continued

\begin{tabular}{|c|c|c|c|c|c|c|c|}
\hline $\begin{array}{c}\text { Point } \\
\text { no. }\end{array}$ & $\begin{array}{c}p \\
(\mathrm{MPa})\end{array}$ & $\begin{array}{c}\rho \\
\left(\mathrm{kg} \mathrm{m}^{-3}\right)\end{array}$ & $\begin{array}{l}T_{\exp } \\
(\mathrm{K})\end{array}$ & $\begin{array}{c}\lambda_{\exp } \\
\left(\mathrm{W} \mathrm{m}^{-1} \mathrm{~K}^{-1}\right)\end{array}$ & STAT & $\begin{array}{l}\text { Relative } \\
\text { dev. }(\%)\end{array}$ & $\begin{array}{c}q \\
\left(\mathrm{~W} \mathrm{~m} \mathrm{~m}^{-1}\right)\end{array}$ \\
\hline \multicolumn{8}{|c|}{ Nominal temperature $340 \mathrm{~K}$} \\
\hline 6195 & 2.0712 & 28.587 & 342.028 & 0.02024 & 0.001 & -1.43 & 0.07948 \\
\hline 6196 & 2.0712 & 28.614 & 341.716 & 0.02016 & 0.001 & -1.78 & 0.07174 \\
\hline 6197 & 2.0713 & 28.642 & 341.421 & 0.02022 & 0.001 & -1.40 & 0.06439 \\
\hline 6198 & 1.3694 & 18.786 & 343.481 & 0.01995 & 0.001 & -2.15 & 0.10968 \\
\hline 6199 & 1.3694 & 18.809 & 343.095 & 0.01993 & 0.001 & -2.17 & 0.10055 \\
\hline 6200 & 1.3694 & 18.829 & 342.723 & 0.01988 & 0.001 & -2.29 & 0.09182 \\
\hline 6201 & 1.3694 & 18.848 & 342.368 & 0.01989 & 0.001 & -2.19 & 0.08350 \\
\hline 6202 & 1.3694 & 18.868 & 342.026 & 0.01992 & 0.001 & -1.93 & 0.07555 \\
\hline 6203 & 1.3695 & 18.888 & 341.699 & 0.01988 & 0.001 & -2.09 & 0.06801 \\
\hline 6204 & 1.3694 & 18.903 & 341.405 & 0.01976 & 0.002 & -2.63 & 0.06086 \\
\hline 6216 & 0.6979 & 9.559 & 343.548 & 0.01966 & 0.001 & -2.61 & 0.10506 \\
\hline 6217 & 0.6979 & 9.567 & 343.146 & 0.01963 & 0.001 & -2.64 & 0.09613 \\
\hline 6218 & 0.6978 & 9.578 & 342.756 & 0.01958 & 0.001 & -2.84 & 0.08761 \\
\hline 6219 & 0.6978 & 9.590 & 342.387 & 0.01959 & 0.001 & -2.66 & 0.07947 \\
\hline 6220 & 0.6979 & 9.602 & 342.037 & 0.01960 & 0.001 & -2.53 & 0.07173 \\
\hline 6221 & 0.6978 & 9.610 & 341.703 & 0.01952 & 0.002 & -2.89 & 0.06439 \\
\hline 6222 & 0.6978 & 9.618 & 341.387 & 0.01955 & 0.002 & -2.64 & 0.05744 \\
\hline 6234 & 0.3486 & 4.764 & 343.883 & 0.01963 & 0.002 & -2.27 & 0.10505 \\
\hline 6235 & 0.3485 & 4.772 & 343.440 & 0.01963 & 0.001 & -2.18 & 0.09612 \\
\hline 6236 & 0.3486 & 4.776 & 343.023 & 0.01966 & 0.002 & -1.94 & 0.08760 \\
\hline 6237 & 0.3485 & 4.779 & 342.632 & 0.01959 & 0.001 & -2.18 & 0.07947 \\
\hline 6238 & 0.3485 & 4.787 & 342.259 & 0.01965 & 0.002 & -1.80 & 0.07173 \\
\hline 6239 & 0.3485 & 4.791 & 341.896 & 0.01959 & 0.002 & -2.02 & 0.06438 \\
\hline 6240 & 0.3486 & 4.799 & 341.572 & 0.01968 & 0.003 & -1.46 & 0.05743 \\
\hline 6252 & 0.1686 & 2.304 & 343.987 & 0.01986 & 0.002 & -0.86 & 0.10052 \\
\hline 6253 & 0.1686 & 2.304 & 343.531 & 0.01986 & 0.002 & -0.76 & 0.09180 \\
\hline 6254 & 0.1686 & 2.308 & 343.096 & 0.01984 & 0.002 & -0.75 & 0.08346 \\
\hline 6255 & 0.1685 & 2.312 & 342.690 & 0.01982 & 0.002 & -0.73 & 0.07553 \\
\hline 6256 & 0.1685 & 2.312 & 342.308 & 0.01986 & 0.002 & -0.44 & 0.06800 \\
\hline 6257 & 0.1685 & 2.316 & 341.932 & 0.01983 & 0.002 & -0.51 & 0.06085 \\
\hline 6258 & 0.1685 & 2.319 & 341.589 & 0.01984 & 0.003 & -0.36 & 0.05410 \\
\hline
\end{tabular}

scales of Figs. 9 and 10 are deliberately the same. Superimposing Figs. 9 and 10 shows that the systematic deviations at low densities are also seen in the steady-state measurements, which is why we ascribe this systematic deviation to a difference in the values for dilute gas thermal conductivity $\lambda_{0}$. The agreement between the transient measurements and the steady-state results is quite good; the mean deviation between the two methods is $1 \%$.

The steady-state single-wire results are given in Table 4 under several different headings. The single-wire experiments are an attempt to measure the end effects in each wire. At the ends of each wire, heat is flowing from the wire ends to the wire supports. In addition, a part of the applied heat is flowing from the end of the wire through the fluid to the cell ends, a geometry quite different from the center portions of the wire. The lines in Table 4 are in pairs by run and point number. The first line is the regular or normal result calculated from the measured bridge imbalances, while the result on the second line uses the voltages measured directly across the individual hot wires. As a check the added voltmeter was also connected across the bridge. In this case the two lines given in Table 4 are virtually identical. Relative deviations of the data from the thermal conductivity surface of argon [12] are also provided in Table 4 . These deviations are shown in Fig. 11 as a function of the applied power $q$. We see that for the short hot wire the thermal conductivity results are about $20 \%$ above the normal steady-state bridge values, while for the long hot wire the deviation is around $4 \%$ for the higher powers. An inspection of the single-wire voltage profiles indicates that turbulent convection (see trace c of Fig. 6) is first seen in the short-wire cell; however, it carries over into the full-bridge measurement. For the low-temperature system [1], using wire lengths of $0.05 \mathrm{~m}$ and 0.10 $\mathrm{m}$, end effects of $8.3 \%$ for the short wire and $4.8 \%$ for the long wire were observed in nitrogen gas. The present 


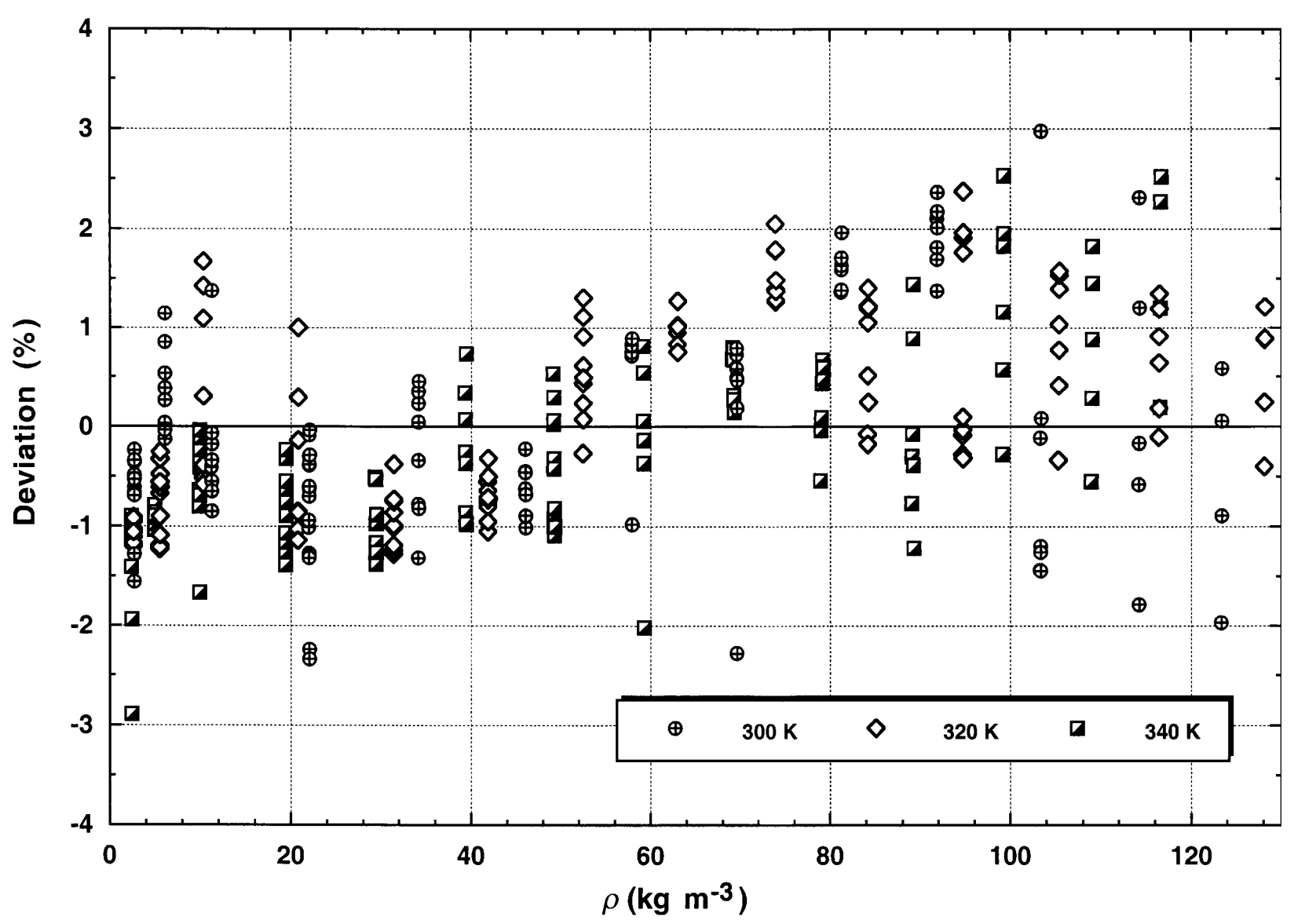

Fig. 10. Steady-state thermal conductivity results for argon. The baseline is the correlation of Younglove and Hanley [12].

wires have lengths of $0.05 \mathrm{~m}$ and $0.20 \mathrm{~m}$, with end effects of $16 \%$ and $4 \%$, respectively. For equivalent wire lengths (short wires), the present end effects are larger by about a factor of 2 , quite reasonable given that the present steady-state measurements run considerably longer in time on a different gas.

The very last segment in Table 4 shows a series of runs made at a pressure considerably below ambient with the added voltmeter connected across the short hot wire. The exact pressure was difficult to determine because we did not have an appropriate pressure gage. We estimate that the pressure must be between $2 \mathrm{~Pa}$ and $1300 \mathrm{~Pa}$, somewhere between the limit of the forepump and the rather approximate reading of the regular pressure gage. We can be certain that these measurements fall into the Knudsen region where the thermal conductivity is proportional to pressure. The deviations for the two highest power levels in this series fall between the ones for the long and the short hot wires. We might expect the end effects to be smaller under these conditions because the heat flow from the wire ends to the cell ends is reduced.
It is shown that for all conditions, except perhaps turbulent convection in either cell, having the short hot wire in the bridge insures sufficient compensation for end effects. This seems to be in agreement with the conclusions of Taxis and Stephan [7]. Finally, the steady-state results, the first line of each pair, are independent of applied power, an excellent verification of the key requirement that the results are free from the influence of natural convection.

\subsection{Analysis}

Argon was selected as the test fluid because the dilute-gas thermal conductivity $\lambda_{0}$ and the first density correction $\lambda_{1}=(\partial \lambda / \partial \rho)_{\mathrm{T}}$ are well known from Chapman-Enskog theory, as is the pair interaction potential for argon. The present results must be compared with the values derived from theory to validate the technique. To make the analysis easier, the large number of points was reduced by averaging as follows. All of the results were shifted from their experimental temperatures to the even temperatures of $300 \mathrm{~K}, 320 \mathrm{~K}$, and $340 \mathrm{~K}$ using the 
Table 3. Thermal conductivity of argon, steady-state method

\begin{tabular}{|c|c|c|c|c|c|c|c|c|c|}
\hline $\begin{array}{c}\text { Point } \\
\text { no. }\end{array}$ & $\begin{array}{c}p \\
(\mathrm{MPa})\end{array}$ & $\begin{array}{c}\rho \\
\left(\mathrm{kg} \mathrm{m}^{-3}\right)\end{array}$ & $\begin{array}{l}T_{\text {exp }} \\
(\mathrm{K})\end{array}$ & $\begin{array}{c}\lambda_{\exp } \\
\left(\mathrm{W} \mathrm{m}^{-1} \mathrm{~K}^{-1}\right)\end{array}$ & $T B A N D$ & $\begin{array}{l}\text { Relative } \\
\text { dev. }(\%)\end{array}$ & $\begin{array}{c}q \\
\left(\mathrm{~W} \mathrm{~m}^{-1}\right)\end{array}$ & $\Delta T$ & $R a$ \\
\hline \multicolumn{10}{|c|}{ Nominal temperature $300 \mathrm{~K}$} \\
\hline 3015 & 7.4443 & 123.423 & 300.647 & 0.02051 & 1.33 & -0.89 & 0.01420 & 0.663 & 51646 \\
\hline 3016 & 7.4439 & 123.407 & 300.667 & 0.02082 & 1.45 & 0.58 & 0.01582 & 0.722 & 56238 \\
\hline 3017 & 7.4442 & 123.391 & 300.713 & 0.02071 & 1.42 & 0.05 & 0.01753 & 0.796 & 61990 \\
\hline 3018 & 7.4439 & 123.363 & 300.757 & 0.02030 & 1.10 & -1.97 & 0.01933 & 0.886 & 68872 \\
\hline 3034 & 6.9070 & 114.295 & 300.621 & 0.02093 & 1.46 & 2.31 & 0.01267 & 0.591 & 39372 \\
\hline 3035 & 6.9071 & 114.283 & 300.651 & 0.02070 & 1.44 & 1.20 & 0.01420 & 0.665 & 44239 \\
\hline 3036 & 6.9069 & 114.263 & 300.694 & 0.02010 & 1.61 & -1.79 & 0.01582 & 0.755 & 50224 \\
\hline 3038 & 6.9073 & 114.235 & 300.766 & 0.02042 & 1.12 & -0.17 & 0.01933 & 0.894 & 59373 \\
\hline 3039 & 6.9074 & 114.215 & 300.812 & 0.02034 & 1.81 & -0.58 & 0.02122 & 0.977 & 64788 \\
\hline 3051 & 6.2656 & 103.318 & 300.866 & 0.02016 & 1.21 & -0.12 & 0.02319 & 1.088 & 58667 \\
\hline 3052 & 6.2652 & 103.326 & 300.830 & 0.01989 & 1.43 & -1.44 & 0.02122 & 1.015 & 54769 \\
\hline 3053 & 6.2653 & 103.345 & 300.788 & 0.01994 & 1.13 & -1.20 & 0.01933 & 0.930 & 50217 \\
\hline 3054 & 6.2652 & 103.357 & 300.747 & 0.01993 & 1.22 & -1.26 & 0.01754 & 0.850 & 45946 \\
\hline 3055 & 6.2654 & 103.377 & 300.709 & 0.01989 & 1.63 & -1.45 & 0.01583 & 0.774 & 41870 \\
\hline 3056 & 6.2654 & 103.393 & 300.672 & 0.02019 & 1.53 & 0.08 & 0.01420 & 0.690 & 37329 \\
\hline 3058 & 6.2654 & 103.425 & 300.592 & 0.02079 & 1.77 & 2.97 & 0.01122 & 0.537 & 29104 \\
\hline 3071 & 5.5847 & 91.824 & 300.867 & 0.02017 & 1.18 & 1.37 & 0.02319 & 1.109 & 46858 \\
\hline 3072 & 5.5847 & 91.836 & 300.831 & 0.02023 & 1.13 & 1.69 & 0.02122 & 1.018 & 43048 \\
\hline 3073 & 5.5848 & 91.856 & 300.783 & 0.02025 & 1.27 & 1.81 & 0.01933 & 0.933 & 39457 \\
\hline 3074 & 5.5848 & 91.872 & 300.738 & 0.02031 & 1.07 & 2.10 & 0.01753 & 0.849 & 35936 \\
\hline 3075 & 5.5848 & 91.884 & 300.700 & 0.02029 & 1.57 & 2.01 & 0.01582 & 0.771 & 32670 \\
\hline 3076 & 5.5847 & 91.896 & 300.663 & 0.02032 & 1.46 & 2.17 & 0.01420 & 0.695 & 29460 \\
\hline 3077 & 5.5846 & 91.908 & 300.625 & 0.02036 & 1.50 & 2.36 & 0.01267 & 0.622 & 26388 \\
\hline 3094 & 4.9524 & 81.270 & 300.629 & 0.02002 & 1.65 & 1.96 & 0.01267 & 0.639 & 21009 \\
\hline 3095 & 4.9525 & 81.258 & 300.670 & 0.01997 & 1.85 & 1.71 & 0.01421 & 0.715 & 23490 \\
\hline 3096 & 4.9525 & 81.246 & 300.709 & 0.01995 & 1.32 & 1.63 & 0.01583 & 0.793 & 26058 \\
\hline 3097 & 4.9526 & 81.234 & 300.751 & 0.01997 & 1.56 & 1.71 & 0.01754 & 0.874 & 28690 \\
\hline 3098 & 4.9526 & 81.222 & 300.797 & 0.01990 & 1.49 & 1.38 & 0.01933 & 0.962 & 31549 \\
\hline 3099 & 4.9527 & 81.210 & 300.845 & 0.01995 & 1.12 & 1.59 & 0.02122 & 1.048 & 34349 \\
\hline 3100 & 4.9528 & 81.198 & 300.887 & 0.01990 & 1.38 & 1.36 & 0.02319 & 1.142 & 37404 \\
\hline 3113 & 4.2565 & 69.625 & 300.610 & 0.01891 & 1.77 & -2.28 & 0.01122 & 0.606 & 14468 \\
\hline 3115 & 4.2560 & 69.601 & 300.674 & 0.01944 & 1.26 & 0.46 & 0.01420 & 0.742 & 17689 \\
\hline 3116 & 4.2561 & 69.589 & 300.727 & 0.01938 & 1.18 & 0.18 & 0.01583 & 0.826 & 19675 \\
\hline 3117 & 4.2561 & 69.581 & 300.764 & 0.01945 & 1.13 & 0.49 & 0.01754 & 0.909 & 21640 \\
\hline 3118 & 4.2562 & 69.569 & 300.812 & 0.01947 & 1.35 & 0.58 & 0.01933 & 0.997 & 23725 \\
\hline 3119 & 4.2562 & 69.561 & 300.846 & 0.01951 & 1.20 & 0.79 & 0.02122 & 1.088 & 25868 \\
\hline 3120 & 4.2561 & 69.545 & 300.902 & 0.01950 & 1.37 & 0.72 & 0.02319 & 1.185 & 28147 \\
\hline 3134 & 3.5546 & 57.937 & 300.649 & 0.01889 & 1.41 & -0.98 & 0.01267 & 0.689 & 11239 \\
\hline 3135 & 3.5547 & 57.929 & 300.680 & 0.01925 & 1.44 & 0.89 & 0.01420 & 0.756 & 12333 \\
\hline 3136 & 3.5546 & 57.917 & 300.725 & 0.01925 & 1.78 & 0.89 & 0.01583 & 0.841 & 13697 \\
\hline 3137 & 3.5545 & 57.909 & 300.770 & 0.01924 & 0.95 & 0.82 & 0.01754 & 0.929 & 15132 \\
\hline 3138 & 3.5544 & 57.897 & 300.814 & 0.01923 & 0.90 & 0.75 & 0.01933 & 1.023 & 16635 \\
\hline 3139 & 3.5544 & 57.885 & 300.865 & 0.01923 & 1.10 & 0.71 & 0.02122 & 1.119 & 18194 \\
\hline 3140 & 3.5544 & 57.877 & 300.910 & 0.01924 & 1.23 & 0.76 & 0.02319 & 1.219 & 19801 \\
\hline 4013 & 2.8395 & 46.096 & 300.725 & 0.01865 & 1.54 & -0.89 & 0.01122 & 0.623 & 6343 \\
\hline 4014 & 2.8395 & 46.092 & 300.758 & 0.01865 & 1.52 & -0.90 & 0.01267 & 0.703 & 7145 \\
\hline 4015 & 2.8395 & 46.084 & 300.807 & 0.01863 & 1.22 & -1.01 & 0.01420 & 0.787 & 7999 \\
\hline 4016 & 2.8395 & 46.076 & 300.844 & 0.01870 & 1.48 & -0.68 & 0.01582 & 0.873 & 8863 \\
\hline 4017 & 2.8395 & 46.068 & 300.897 & 0.01871 & 0.89 & -0.62 & 0.01753 & 0.965 & 9788 \\
\hline 4018 & 2.8394 & 46.060 & 300.938 & 0.01874 & 0.91 & -0.46 & 0.01933 & 1.060 & 10745 \\
\hline 4019 & 2.8392 & 46.048 & 300.987 & 0.01875 & 0.95 & -0.45 & 0.02121 & 1.161 & 11757 \\
\hline 4020 & 2.8392 & 46.040 & 301.037 & 0.01879 & 1.12 & -0.23 & 0.02319 & 1.264 & 12786 \\
\hline 4033 & 2.1134 & 34.180 & 300.707 & 0.01864 & 1.92 & 0.45 & 0.01123 & 0.627 & 3453 \\
\hline 4034 & 2.1132 & 34.172 & 300.753 & 0.01841 & 1.89 & -0.82 & 0.01267 & 0.716 & 3941 \\
\hline 4035 & 2.1133 & 34.168 & 300.795 & 0.01863 & 1.40 & 0.35 & 0.01421 & 0.793 & 4360 \\
\hline 4036 & 2.1132 & 34.160 & 300.840 & 0.01861 & 1.24 & 0.23 & 0.01583 & 0.884 & 4855 \\
\hline
\end{tabular}


Table 3. Thermal conductivity of argon, steady-state method—Continued

\begin{tabular}{|c|c|c|c|c|c|c|c|c|c|}
\hline $\begin{array}{c}\text { Point } \\
\text { no. }\end{array}$ & $\begin{array}{c}p \\
(\mathrm{MPa})\end{array}$ & $\begin{array}{c}\rho \\
\left(\mathrm{kg} \mathrm{m}^{-3}\right)\end{array}$ & $\begin{array}{l}T_{\exp } \\
(\mathrm{K})\end{array}$ & $\left(\mathrm{W} \mathrm{m}^{-1} \mathrm{~K}^{-1}\right)$ & $T B A N D$ & $\begin{array}{l}\text { Relative } \\
\text { dev. (\%) }\end{array}$ & $\begin{array}{c}q \\
\left(\mathrm{~W} \mathrm{~m}^{-1}\right)\end{array}$ & $\Delta T$ & $R a$ \\
\hline \multicolumn{10}{|c|}{ Nominal temperature $300 \mathrm{~K}$} \\
\hline 4037 & 2.1132 & 34.152 & 300.885 & 0.01858 & 1.48 & 0.04 & 0.01754 & 0.980 & 5379 \\
\hline 4038 & 2.1132 & 34.148 & 300.932 & 0.01851 & 0.95 & -0.34 & 0.01934 & 1.084 & 5942 \\
\hline 4039 & 2.1132 & 34.140 & 300.990 & 0.01843 & 0.85 & -0.78 & 0.02123 & 1.193 & 6535 \\
\hline 4040 & 2.1132 & 34.132 & 301.050 & 0.01833 & 0.99 & -1.32 & 0.02320 & 1.309 & 7164 \\
\hline 4053 & 1.3696 & 22.059 & 300.691 & 0.01830 & 1.67 & -0.04 & 0.01123 & 0.641 & 1445 \\
\hline 4054 & 1.3697 & 22.055 & 300.739 & 0.01789 & 1.76 & -2.34 & 0.01267 & 0.740 & 1667 \\
\hline 4055 & 1.3696 & 22.051 & 300.789 & 0.01791 & 1.31 & -2.24 & 0.01421 & 0.829 & 1864 \\
\hline 4056 & 1.3698 & 22.051 & 300.822 & 0.01826 & 1.17 & -0.29 & 0.01583 & 0.905 & 2036 \\
\hline 4057 & 1.3696 & 22.047 & 300.869 & 0.01830 & 0.82 & -0.09 & 0.01754 & 1.001 & 2248 \\
\hline 4058 & 1.3698 & 22.043 & 300.922 & 0.01826 & 0.86 & -0.30 & 0.01934 & 1.105 & 2481 \\
\hline 4059 & 1.3698 & 22.043 & 300.975 & 0.01825 & 0.75 & -0.38 & 0.02122 & 1.213 & 2721 \\
\hline 4060 & 1.3698 & 22.035 & 301.030 & 0.01825 & 1.04 & -0.39 & 0.02320 & 1.325 & 2970 \\
\hline 4061 & 1.3700 & 22.035 & 301.091 & 0.01826 & 0.52 & -0.38 & 0.02526 & 1.442 & 3229 \\
\hline 4062 & 1.3698 & 22.027 & 301.147 & 0.01822 & 0.66 & -0.60 & 0.02741 & 1.567 & 3504 \\
\hline 4063 & 1.3698 & 22.023 & 301.211 & 0.01821 & 0.59 & -0.70 & 0.02964 & 1.695 & 3788 \\
\hline 4064 & 1.3699 & 22.019 & 301.275 & 0.01822 & 0.61 & -0.62 & 0.03197 & 1.825 & 4075 \\
\hline 4065 & 1.3699 & 22.015 & 301.353 & 0.01810 & 0.67 & -1.32 & 0.03438 & 1.975 & 4404 \\
\hline 4066 & 1.3699 & 22.007 & 301.417 & 0.01811 & 0.57 & -1.29 & 0.03688 & 2.116 & 4714 \\
\hline 4067 & 1.3700 & 22.003 & 301.494 & 0.01812 & 0.45 & -1.27 & 0.03947 & 2.262 & 5034 \\
\hline 4068 & 1.3701 & 21.999 & 301.569 & 0.01811 & 0.50 & -1.31 & 0.04214 & 2.415 & 5367 \\
\hline 4069 & 1.3699 & 21.991 & 301.641 & 0.01818 & 0.46 & -0.94 & 0.04491 & 2.562 & 5686 \\
\hline 4070 & 1.3700 & 21.987 & 301.724 & 0.01817 & 0.46 & -1.01 & 0.04776 & 2.724 & 6039 \\
\hline 4072 & 0.7011 & 11.249 & 300.661 & 0.01834 & 1.79 & 1.37 & 0.01123 & 0.641 & 369 \\
\hline 4073 & 0.7010 & 11.245 & 300.753 & 0.01794 & 1.36 & -0.85 & 0.01421 & 0.829 & 477 \\
\hline 4075 & 0.7012 & 11.241 & 300.957 & 0.01799 & 1.24 & -0.65 & 0.02122 & 1.235 & 708 \\
\hline 4076 & 0.7010 & 11.229 & 301.197 & 0.01802 & 0.59 & -0.55 & 0.02964 & 1.722 & 983 \\
\hline 4077 & 0.7009 & 11.221 & 301.325 & 0.01811 & 0.47 & -0.07 & 0.03438 & 1.986 & 1131 \\
\hline 4078 & 0.7010 & 11.217 & 301.470 & 0.01810 & 0.53 & -0.18 & 0.03947 & 2.281 & 1297 \\
\hline 4079 & 0.7011 & 11.213 & 301.632 & 0.01807 & 0.39 & -0.34 & 0.04491 & 2.597 & 1473 \\
\hline 4080 & 0.7011 & 11.205 & 301.799 & 0.01807 & 0.34 & -0.41 & 0.05070 & 2.932 & 1658 \\
\hline 4093 & 0.3781 & 6.056 & 300.717 & 0.01820 & 1.87 & 1.14 & 0.01421 & 0.818 & 135 \\
\hline 4094 & 0.3780 & 6.052 & 300.816 & 0.01815 & 1.06 & 0.85 & 0.01754 & 1.013 & 167 \\
\hline 4095 & 0.3780 & 6.048 & 300.919 & 0.01810 & 0.84 & 0.53 & 0.02122 & 1.229 & 202 \\
\hline 4096 & 0.3780 & 6.044 & 301.043 & 0.01807 & 0.72 & 0.38 & 0.02526 & 1.464 & 241 \\
\hline 4097 & 0.3780 & 6.044 & 301.170 & 0.01806 & 0.53 & 0.26 & 0.02964 & 1.720 & 282 \\
\hline 4098 & 0.3780 & 6.040 & 301.307 & 0.01802 & 0.39 & 0.03 & 0.03438 & 1.998 & 327 \\
\hline 4099 & 0.3780 & 6.040 & 301.447 & 0.01802 & 0.32 & -0.04 & 0.03947 & 2.294 & 375 \\
\hline 4100 & 0.3781 & 6.036 & 301.614 & 0.01801 & 0.28 & -0.13 & 0.04491 & 2.612 & 426 \\
\hline 4111 & 0.1684 & 2.692 & 300.811 & 0.01765 & 0.95 & -1.56 & 0.01754 & 1.041 & 34 \\
\hline 4112 & 0.1685 & 2.692 & 300.920 & 0.01771 & 0.79 & -1.28 & 0.02122 & 1.256 & 41 \\
\hline 4113 & 0.1686 & 2.692 & 301.025 & 0.01790 & 0.68 & -0.24 & 0.02526 & 1.479 & 48 \\
\hline 4114 & 0.1686 & 2.692 & 301.159 & 0.01789 & 0.56 & -0.34 & 0.02964 & 1.737 & 56 \\
\hline 4115 & 0.1685 & 2.689 & 301.295 & 0.01789 & 0.42 & -0.36 & 0.03438 & 2.014 & 65 \\
\hline 4116 & 0.1684 & 2.689 & 301.447 & 0.01787 & 0.32 & -0.49 & 0.03946 & 2.314 & 74 \\
\hline 4117 & 0.1685 & 2.689 & 301.601 & 0.01787 & 0.36 & -0.53 & 0.04490 & 2.633 & 85 \\
\hline 4118 & 0.1684 & 2.685 & 301.772 & 0.01787 & 0.22 & -0.61 & 0.05070 & 2.974 & 95 \\
\hline 4119 & 0.1685 & 2.685 & 301.958 & 0.01787 & 0.16 & -0.66 & 0.05684 & 3.333 & 106 \\
\hline 4120 & 0.1686 & 2.685 & 302.138 & 0.01787 & 0.20 & -0.69 & 0.06333 & 3.713 & 118 \\
\hline \multicolumn{10}{|c|}{ Nominal temperature $320 \mathrm{~K}$} \\
\hline 5011 & 8.3286 & 128.181 & 320.414 & 0.02219 & 1.42 & 1.21 & 0.01519 & 0.662 & 45411 \\
\hline 5012 & 8.3287 & 128.165 & 320.448 & 0.02212 & 1.07 & 0.89 & 0.01693 & 0.734 & 50310 \\
\hline 5013 & 8.3287 & 128.149 & 320.483 & 0.02212 & 1.37 & 0.88 & 0.01876 & 0.807 & 55252 \\
\hline 5014 & 8.3288 & 128.133 & 320.524 & 0.02198 & 1.06 & 0.24 & 0.02068 & 0.887 & 60707 \\
\hline 5015 & 8.3287 & 128.109 & 320.566 & 0.02184 & 1.02 & -0.40 & 0.02270 & 0.970 & 66368 \\
\hline
\end{tabular}


Table 3. Thermal conductivity of argon, steady-state method-Continued

\begin{tabular}{|c|c|c|c|c|c|c|c|c|c|}
\hline $\begin{array}{c}\text { Point } \\
\text { no. }\end{array}$ & $\begin{array}{c}p \\
(\mathrm{MPa})\end{array}$ & $\begin{array}{c}\rho \\
\left(\mathrm{kg} \mathrm{m}^{-3}\right)\end{array}$ & $\begin{array}{l}T_{\exp } \\
(\mathrm{K})\end{array}$ & $\begin{array}{c}\lambda_{\exp } \\
\left(\mathrm{W} \mathrm{m}^{-1} \mathrm{~K}^{-1}\right)\end{array}$ & $T B A N D$ & $\begin{array}{l}\text { Relative } \\
\text { dev. (\%) }\end{array}$ & $\begin{array}{c}q \\
\left(\mathrm{~W} \mathrm{~m}^{-1}\right)\end{array}$ & $\Delta T$ & $R a$ \\
\hline \multicolumn{10}{|c|}{ Nominal temperature $320 \mathrm{~K}$} \\
\hline 5031 & 7.5812 & 116.492 & 320.447 & 0.02190 & 1.34 & 1.34 & 0.01693 & 0.752 & 42454 \\
\hline 5032 & 7.5812 & 116.480 & 320.478 & 0.02187 & 1.06 & 1.19 & 0.01876 & 0.828 & 46742 \\
\hline 5033 & 7.5813 & 116.460 & 320.521 & 0.02181 & 0.79 & 0.91 & 0.02068 & 0.908 & 51240 \\
\hline 5034 & 7.5815 & 116.444 & 320.564 & 0.02175 & 1.32 & 0.64 & 0.02270 & 0.992 & 55906 \\
\hline 5035 & 7.5815 & 116.428 & 320.604 & 0.02165 & 0.92 & 0.18 & 0.02480 & 1.080 & 60833 \\
\hline 5036 & 7.5816 & 116.412 & 320.644 & 0.02159 & 0.85 & -0.11 & 0.02701 & 1.169 & 65840 \\
\hline 5051 & 6.8718 & 105.431 & 320.434 & 0.02165 & 1.12 & 1.57 & 0.01693 & 0.769 & 35481 \\
\hline 5052 & 6.8721 & 105.419 & 320.474 & 0.02164 & 1.04 & 1.53 & 0.01876 & 0.847 & 39058 \\
\hline 5053 & 6.8719 & 105.403 & 320.510 & 0.02161 & 0.90 & 1.39 & 0.02068 & 0.930 & 42813 \\
\hline 5054 & 6.8720 & 105.383 & 320.564 & 0.02154 & 1.28 & 1.03 & 0.02269 & 1.017 & 46793 \\
\hline 5055 & 6.8722 & 105.367 & 320.607 & 0.02148 & 0.94 & 0.77 & 0.02480 & 1.106 & 50882 \\
\hline 5056 & 6.8722 & 105.355 & 320.644 & 0.02141 & 0.83 & 0.41 & 0.02701 & 1.200 & 55164 \\
\hline 5059 & 6.8724 & 105.299 & 320.793 & 0.02125 & 0.98 & -0.34 & 0.03418 & 1.497 & 68604 \\
\hline 5071 & 6.1864 & 94.785 & 320.337 & 0.02154 & 1.90 & 2.37 & 0.01355 & 0.632 & 23485 \\
\hline 5072 & 6.1863 & 94.769 & 320.377 & 0.02145 & 1.25 & 1.96 & 0.01519 & 0.708 & 26285 \\
\hline 5073 & 6.1864 & 94.757 & 320.422 & 0.02144 & 1.29 & 1.91 & 0.01693 & 0.785 & 29134 \\
\hline 5074 & 6.1863 & 94.745 & 320.459 & 0.02141 & 1.24 & 1.76 & 0.01876 & 0.867 & 32135 \\
\hline 5075 & 6.1864 & 94.725 & 320.510 & 0.02097 & 1.17 & -0.32 & 0.02068 & 0.970 & 35906 \\
\hline 5076 & 6.1863 & 94.709 & 320.556 & 0.02105 & 1.14 & 0.09 & 0.02269 & 1.054 & 39002 \\
\hline 5077 & 6.1863 & 94.697 & 320.596 & 0.02103 & 0.85 & -0.04 & 0.02480 & 1.147 & 42405 \\
\hline 5078 & 6.1861 & 94.677 & 320.645 & 0.02103 & 0.88 & -0.03 & 0.02701 & 1.241 & 45850 \\
\hline 5079 & 6.1860 & 94.661 & 320.689 & 0.02102 & 1.00 & -0.09 & 0.02930 & 1.339 & 49437 \\
\hline 5080 & 6.1860 & 94.641 & 320.741 & 0.02099 & 0.78 & -0.28 & 0.03169 & 1.442 & 53179 \\
\hline 5091 & 5.5066 & 84.234 & 320.284 & 0.02101 & 1.96 & 1.21 & 0.01200 & 0.581 & 16950 \\
\hline 5092 & 5.5067 & 84.222 & 320.324 & 0.02080 & 1.92 & 0.24 & 0.01355 & 0.659 & 19231 \\
\hline 5093 & 5.5068 & 84.210 & 320.360 & 0.02105 & 1.38 & 1.40 & 0.01519 & 0.728 & 21218 \\
\hline 5094 & 5.5065 & 84.194 & 320.403 & 0.02101 & 1.09 & 1.19 & 0.01693 & 0.809 & 23571 \\
\hline 5095 & 5.5066 & 84.186 & 320.437 & 0.02102 & 1.41 & 1.22 & 0.01875 & 0.893 & 25981 \\
\hline 5096 & 5.5068 & 84.174 & 320.483 & 0.02102 & 1.14 & 1.22 & 0.02068 & 0.980 & 28495 \\
\hline 5097 & 5.5067 & 84.158 & 320.530 & 0.02099 & 0.97 & 1.05 & 0.02269 & 1.072 & 31151 \\
\hline 5098 & 5.5070 & 84.150 & 320.573 & 0.02087 & 1.06 & 0.51 & 0.02480 & 1.172 & 34039 \\
\hline 5099 & 5.5071 & 84.138 & 320.627 & 0.02073 & 1.05 & -0.18 & 0.02700 & 1.278 & 37084 \\
\hline 5100 & 5.5070 & 84.119 & 320.682 & 0.02075 & 1.03 & -0.08 & 0.02930 & 1.378 & 39960 \\
\hline 5112 & 4.8436 & 73.944 & 320.298 & 0.02081 & 1.50 & 1.48 & 0.01355 & 0.665 & 14844 \\
\hline 5113 & 4.8438 & 73.940 & 320.334 & 0.02078 & 1.77 & 1.37 & 0.01519 & 0.743 & 16599 \\
\hline 5114 & 4.8438 & 73.932 & 320.369 & 0.02077 & 1.07 & 1.28 & 0.01693 & 0.826 & 18436 \\
\hline 5115 & 4.8438 & 73.916 & 320.420 & 0.02077 & 1.08 & 1.27 & 0.01876 & 0.912 & 20342 \\
\hline 5116 & 4.8439 & 73.908 & 320.463 & 0.02077 & 0.98 & 1.26 & 0.02068 & 1.002 & 22330 \\
\hline 5117 & 4.8438 & 73.892 & 320.506 & 0.02080 & 1.17 & 1.39 & 0.02269 & 1.095 & 24367 \\
\hline 5118 & 4.8440 & 73.884 & 320.554 & 0.02088 & 1.13 & 1.79 & 0.02480 & 1.187 & 26408 \\
\hline 5119 & 4.8441 & 73.876 & 320.597 & 0.02088 & 1.44 & 1.78 & 0.02700 & 1.287 & 28623 \\
\hline 5120 & 4.8442 & 73.864 & 320.650 & 0.02094 & 1.17 & 2.05 & 0.02930 & 1.388 & 30823 \\
\hline 5133 & 4.1375 & 63.018 & 320.337 & 0.02050 & 1.40 & 1.27 & 0.01519 & 0.760 & 12223 \\
\hline 5134 & 4.1375 & 63.010 & 320.384 & 0.02044 & 1.31 & 1.01 & 0.01693 & 0.847 & 13608 \\
\hline 5135 & 4.1375 & 62.998 & 320.437 & 0.02039 & 1.13 & 0.75 & 0.01876 & 0.938 & 15063 \\
\hline 5136 & 4.1377 & 62.994 & 320.471 & 0.02045 & 1.20 & 1.02 & 0.02068 & 1.029 & 16509 \\
\hline 5137 & 4.1377 & 62.982 & 320.521 & 0.02041 & 0.92 & 0.83 & 0.02269 & 1.128 & 18085 \\
\hline 5138 & 4.1376 & 62.970 & 320.572 & 0.02041 & 1.54 & 0.83 & 0.02480 & 1.229 & 19691 \\
\hline 5139 & 4.1375 & 62.954 & 320.633 & 0.02044 & 1.12 & 0.95 & 0.02700 & 1.332 & 21327 \\
\hline 5140 & 4.1373 & 62.942 & 320.679 & 0.02046 & 1.39 & 1.01 & 0.02930 & 1.440 & 23035 \\
\hline 5152 & 3.4547 & 52.508 & 320.300 & 0.02025 & 1.74 & 1.30 & 0.01355 & 0.692 & 7658 \\
\hline 5153 & 3.4547 & 52.500 & 320.342 & 0.02021 & 1.41 & 1.11 & 0.01519 & 0.776 & 8580 \\
\hline 5154 & 3.4548 & 52.496 & 320.386 & 0.02017 & 0.99 & 0.91 & 0.01693 & 0.864 & 9555 \\
\hline 5155 & 3.4549 & 52.488 & 320.434 & 0.02009 & 0.95 & 0.49 & 0.01875 & 0.960 & 10602 \\
\hline 5156 & 3.4551 & 52.484 & 320.479 & 0.02012 & 1.11 & 0.61 & 0.02068 & 1.055 & 11644 \\
\hline 5157 & 3.4549 & 52.468 & 320.537 & 0.02001 & 1.07 & 0.07 & 0.02269 & 1.161 & 12807 \\
\hline
\end{tabular}


Table 3. Thermal conductivity of argon, steady-state method—Continued

\begin{tabular}{|c|c|c|c|c|c|c|c|c|}
\hline $\begin{array}{l}\text { Point } \\
\text { no. }\end{array}$ & $\begin{array}{c}p \\
(\mathrm{MPa})\end{array}$ & $\begin{array}{c}\rho \\
\left(\mathrm{kg} \mathrm{m}^{-3}\right)\end{array}$ & $\begin{array}{l}T_{\exp } \\
(\mathrm{K})\end{array}$ & $\left(\begin{array}{c}\lambda_{\exp } \\
\left(\mathrm{W} \mathrm{m}^{-1} \mathrm{~K}^{-1}\right)\end{array}\right.$ & TBAND & $\begin{array}{l}\text { Relative } \\
\text { dev. }(\%)\end{array}$ & $\left(\begin{array}{c}q \\
\left(\mathrm{~W}^{-1}\right)\end{array}\right.$ & $\Delta T$ \\
\hline
\end{tabular}

\begin{tabular}{|c|c|c|c|c|c|c|c|c|c|}
\hline \multicolumn{10}{|c|}{ Nominal temperature $320 \mathrm{~K}$} \\
\hline 5158 & 3.4551 & 52.464 & 320.584 & 0.02004 & 1.47 & 0.23 & 0.02480 & 1.264 & 13936 \\
\hline 5159 & 3.4549 & 52.452 & 320.641 & 0.02009 & 0.75 & 0.43 & 0.02700 & 1.371 & 15094 \\
\hline 5160 & 3.4552 & 52.444 & 320.702 & 0.01995 & 1.06 & -0.27 & 0.02930 & 1.494 & 16438 \\
\hline 5171 & 2.7635 & 41.893 & 320.349 & 0.01968 & 1.43 & -0.32 & 0.01519 & 0.801 & 5582 \\
\hline 5172 & 2.7635 & 41.889 & 320.396 & 0.01965 & 1.00 & -0.50 & 0.01693 & 0.893 & 6219 \\
\hline 5173 & 2.7635 & 41.881 & 320.444 & 0.01961 & 1.07 & -0.71 & 0.01875 & 0.990 & 6891 \\
\hline 5174 & 2.7635 & 41.873 & 320.491 & 0.01961 & 1.04 & -0.73 & 0.02067 & 1.090 & 7581 \\
\hline 5175 & 2.7635 & 41.866 & 320.552 & 0.01957 & 0.80 & -0.95 & 0.02269 & 1.197 & 8319 \\
\hline 5176 & 2.7635 & 41.862 & 320.597 & 0.01955 & 0.78 & -1.05 & 0.02480 & 1.308 & 9081 \\
\hline 5177 & 2.7637 & 41.854 & 320.653 & 0.01960 & 0.80 & -0.80 & 0.02700 & 1.418 & 9841 \\
\hline 5178 & 2.7638 & 41.846 & 320.719 & 0.01961 & 1.14 & -0.76 & 0.02930 & 1.536 & 10646 \\
\hline 5179 & 2.7636 & 41.838 & 320.775 & 0.01964 & 1.30 & -0.64 & 0.03169 & 1.657 & 11471 \\
\hline 5180 & 2.7637 & 41.830 & 320.833 & 0.01966 & 1.15 & -0.55 & 0.03418 & 1.782 & 12328 \\
\hline 5182 & 2.0779 & 31.419 & 320.394 & 0.01944 & 1.20 & -0.38 & 0.01693 & 0.907 & 3514 \\
\hline 5183 & 2.0781 & 31.415 & 320.444 & 0.01937 & 1.26 & -0.73 & 0.01876 & 1.008 & 3901 \\
\hline 5184 & 2.0781 & 31.411 & 320.495 & 0.01937 & 0.92 & -0.75 & 0.02068 & 1.110 & 4295 \\
\hline 5185 & 2.0781 & 31.403 & 320.549 & 0.01935 & 0.81 & -0.86 & 0.02269 & 1.219 & 4710 \\
\hline 5186 & 2.0782 & 31.399 & 320.606 & 0.01933 & 0.86 & -0.99 & 0.02480 & 1.332 & 5146 \\
\hline 5187 & 2.0782 & 31.395 & 320.663 & 0.01933 & 0.55 & -1.01 & 0.02700 & 1.450 & 5594 \\
\hline 5188 & 2.0783 & 31.391 & 320.729 & 0.01930 & 0.64 & -1.19 & 0.02930 & 1.574 & 6070 \\
\hline 5189 & 2.0783 & 31.383 & 320.785 & 0.01929 & 0.58 & -1.24 & 0.03169 & 1.702 & 6555 \\
\hline 5190 & 2.0783 & 31.375 & 320.850 & 0.01929 & 0.64 & -1.28 & 0.03417 & 1.834 & 7057 \\
\hline 5203 & 1.3798 & 20.813 & 320.327 & 0.01947 & 1.39 & 1.00 & 0.01519 & 0.816 & 1370 \\
\hline 5204 & 1.3798 & 20.805 & 320.434 & 0.01934 & 1.14 & 0.29 & 0.01875 & 1.013 & 1699 \\
\hline 5205 & 1.3796 & 20.797 & 320.538 & 0.01926 & 0.81 & -0.14 & 0.02269 & 1.230 & 2059 \\
\hline 5207 & 1.3798 & 20.781 & 320.791 & 0.01913 & 0.83 & -0.86 & 0.03169 & 1.727 & 2880 \\
\hline 5208 & 1.3798 & 20.773 & 320.925 & 0.01909 & 0.69 & -1.14 & 0.03675 & 2.006 & 3338 \\
\hline 5209 & 1.3800 & 20.765 & 321.070 & 0.01915 & 0.47 & -0.85 & 0.04218 & 2.293 & 3809 \\
\hline 5210 & 1.3798 & 20.753 & 321.229 & 0.01912 & 0.44 & -1.03 & 0.04799 & 2.610 & 4324 \\
\hline 5222 & 0.6847 & 10.303 & 320.238 & 0.01937 & 1.94 & 1.67 & 0.01200 & 0.649 & 264 \\
\hline 5223 & 0.6849 & 10.303 & 320.324 & 0.01933 & 1.23 & 1.42 & 0.01519 & 0.823 & 334 \\
\hline 5224 & 0.6849 & 10.299 & 320.418 & 0.01927 & 1.26 & 1.09 & 0.01875 & 1.019 & 413 \\
\hline 5225 & 0.6848 & 10.295 & 320.528 & 0.01912 & 0.78 & 0.30 & 0.02269 & 1.243 & 503 \\
\hline 5226 & 0.6849 & 10.291 & 320.656 & 0.01900 & 1.01 & -0.39 & 0.02700 & 1.488 & 601 \\
\hline 5227 & 0.6847 & 10.283 & 320.784 & 0.01898 & 0.80 & -0.52 & 0.03169 & 1.748 & 704 \\
\hline 5228 & 0.6849 & 10.283 & 320.930 & 0.01897 & 0.42 & -0.58 & 0.03675 & 2.027 & 816 \\
\hline 5229 & 0.6848 & 10.275 & 321.074 & 0.01900 & 0.69 & -0.49 & 0.04218 & 2.324 & 932 \\
\hline 5230 & 0.6849 & 10.275 & 321.232 & 0.01901 & 0.48 & -0.45 & 0.04799 & 2.641 & 1058 \\
\hline 5244 & 0.3681 & 5.529 & 320.427 & 0.01875 & 1.06 & -1.09 & 0.01875 & 1.048 & 122 \\
\hline 5245 & 0.3680 & 5.525 & 320.532 & 0.01880 & 1.25 & -0.90 & 0.02269 & 1.265 & 146 \\
\hline 5246 & 0.3680 & 5.525 & 320.645 & 0.01892 & 1.29 & -0.26 & 0.02700 & 1.495 & 173 \\
\hline 5247 & 0.3680 & 5.521 & 320.776 & 0.01891 & 0.79 & -0.33 & 0.03169 & 1.755 & 203 \\
\hline 5248 & 0.3680 & 5.517 & 320.911 & 0.01889 & 0.81 & -0.48 & 0.03675 & 2.038 & 235 \\
\hline 5249 & 0.3680 & 5.517 & 321.067 & 0.01888 & 0.60 & -0.56 & 0.04218 & 2.340 & 269 \\
\hline 5250 & 0.3680 & 5.513 & 321.230 & 0.01888 & 0.74 & -0.61 & 0.04799 & 2.662 & 305 \\
\hline 5261 & 0.3679 & 5.505 & 321.489 & 0.01888 & 0.67 & -0.67 & 0.05741 & 3.185 & 363 \\
\hline 5262 & 0.3680 & 5.501 & 321.774 & 0.01880 & 0.58 & -1.21 & 0.06767 & 3.770 & 429 \\
\hline 5263 & 0.3680 & 5.497 & 322.089 & 0.01882 & 0.65 & -1.19 & 0.07877 & 4.384 & 496 \\
\hline 5264 & 0.3680 & 5.493 & 322.413 & 0.01883 & 0.60 & -1.23 & 0.09071 & 5.045 & 568 \\
\hline 5265 & 0.1734 & 2.601 & 320.774 & 0.01872 & 0.65 & -1.06 & 0.03169 & 1.774 & 45 \\
\hline 5266 & 0.1733 & 2.597 & 320.913 & 0.01870 & 0.31 & -1.20 & 0.03675 & 2.060 & 52 \\
\hline 5267 & 0.1734 & 2.597 & 321.063 & 0.01871 & 0.28 & -1.20 & 0.04218 & 2.363 & 60 \\
\hline 5268 & 0.1734 & 2.597 & 321.227 & 0.01872 & 0.29 & -1.17 & 0.04799 & 2.687 & 68 \\
\hline 5269 & 0.1733 & 2.593 & 321.396 & 0.01877 & 0.21 & -0.92 & 0.05418 & 3.024 & 76 \\
\hline 5270 & 0.1732 & 2.589 & 321.582 & 0.01879 & 0.13 & -0.91 & 0.06073 & 3.388 & 85 \\
\hline 5271 & 0.1733 & 2.589 & 321.769 & 0.01879 & 0.19 & -0.95 & 0.06767 & 3.775 & 95 \\
\hline
\end{tabular}


Table 3. Thermal conductivity of argon, steady-state method—Continued

\begin{tabular}{|c|c|c|c|c|c|c|c|c|c|}
\hline $\begin{array}{c}\text { Point } \\
\text { no. }\end{array}$ & $\begin{array}{c}p \\
(\mathrm{MPa})\end{array}$ & $\begin{array}{c}\rho \\
\left(\mathrm{kg} \mathrm{m}^{-3}\right)\end{array}$ & $\begin{array}{l}T_{\exp } \\
(\mathrm{K})\end{array}$ & $\left(\mathrm{W} \mathrm{m}^{-1} \mathrm{~K}^{-1}\right)$ & $T B A N D$ & $\begin{array}{l}\text { Relative } \\
\text { dev. (\%) }\end{array}$ & $\left(\begin{array}{c}q \\
\left(\mathrm{~W}^{-1}\right)\end{array}\right.$ & $\Delta T$ & $R a$ \\
\hline \multicolumn{10}{|c|}{ Nominal temperature $320 \mathrm{~K}$} \\
\hline 5272 & 0.1733 & 2.589 & 321.972 & 0.01878 & 0.13 & -1.03 & 0.07497 & 4.184 & 105 \\
\hline 5273 & 0.1732 & 2.585 & 322.196 & 0.01879 & 0.11 & -1.03 & 0.08265 & 4.609 & 115 \\
\hline 5274 & 0.1733 & 2.585 & 322.410 & 0.01879 & 0.17 & -1.11 & 0.09071 & 5.060 & 126 \\
\hline \multicolumn{10}{|c|}{ Nominal temperature $340 \mathrm{~K}$} \\
\hline 6008 & 8.1066 & 116.696 & 339.086 & 0.02319 & 1.78 & 2.52 & 0.01524 & 0.652 & 31009 \\
\hline 6009 & 8.1065 & 116.660 & 339.178 & 0.02313 & 0.99 & 2.27 & 0.01991 & 0.840 & 39919 \\
\hline 6010 & 8.1065 & 116.616 & 339.287 & 0.02289 & 0.95 & 1.20 & 0.02519 & 1.056 & 50097 \\
\hline 6011 & 8.1063 & 116.564 & 339.407 & 0.02266 & 0.71 & 0.19 & 0.03110 & 1.293 & 61186 \\
\hline 6026 & 7.5849 & 109.126 & 339.099 & 0.02281 & 1.87 & 1.82 & 0.01524 & 0.666 & 27701 \\
\hline 6027 & 7.5850 & 109.094 & 339.192 & 0.02273 & 1.12 & 1.45 & 0.01991 & 0.861 & 35747 \\
\hline 6028 & 7.5849 & 109.050 & 339.305 & 0.02260 & 0.82 & 0.88 & 0.02519 & 1.079 & 44718 \\
\hline 6029 & 7.5848 & 109.006 & 339.427 & 0.02247 & 0.86 & 0.28 & 0.03110 & 1.318 & 54503 \\
\hline 6030 & 7.5849 & 108.958 & 339.556 & 0.02229 & 0.54 & -0.55 & 0.03762 & 1.579 & 65176 \\
\hline 6044 & 6.9067 & 99.287 & 339.107 & 0.02271 & 1.83 & 2.53 & 0.01524 & 0.675 & 23177 \\
\hline 6045 & 6.9067 & 99.255 & 339.209 & 0.02258 & 1.21 & 1.95 & 0.01991 & 0.876 & 30031 \\
\hline 6046 & 6.9070 & 99.223 & 339.317 & 0.02255 & 1.04 & 1.83 & 0.02519 & 1.095 & 37496 \\
\hline 6047 & 6.9070 & 99.179 & 339.450 & 0.02241 & 0.75 & 1.16 & 0.03110 & 1.342 & 45834 \\
\hline 6048 & 6.9070 & 99.135 & 339.582 & 0.02228 & 0.70 & 0.57 & 0.03763 & 1.608 & 54818 \\
\hline 6049 & 6.9067 & 99.083 & 339.726 & 0.02210 & 0.80 & -0.28 & 0.04477 & 1.898 & 64562 \\
\hline 6062 & 6.2157 & 89.268 & 339.122 & 0.02160 & 1.92 & -1.22 & 0.01524 & 0.714 & 19754 \\
\hline 6063 & 6.2156 & 89.232 & 339.235 & 0.02179 & 1.31 & -0.39 & 0.01991 & 0.915 & 25283 \\
\hline 6064 & 6.2156 & 89.204 & 339.340 & 0.02220 & 0.82 & 1.44 & 0.02519 & 1.125 & 31042 \\
\hline 6065 & 6.2153 & 89.164 & 339.462 & 0.02208 & 0.89 & 0.89 & 0.03110 & 1.380 & 37987 \\
\hline 6066 & 6.2155 & 89.120 & 339.610 & 0.02187 & 0.83 & -0.08 & 0.03762 & 1.663 & 45688 \\
\hline 6067 & 6.2156 & 89.080 & 339.753 & 0.02183 & 0.90 & -0.30 & 0.04477 & 1.956 & 53623 \\
\hline 6068 & 6.2156 & 89.032 & 339.914 & 0.02173 & 0.71 & -0.77 & 0.05254 & 2.273 & 62158 \\
\hline 6080 & 5.5216 & 79.213 & 339.130 & 0.02173 & 1.57 & 0.59 & 0.01524 & 0.715 & 15518 \\
\hline 6081 & 5.5217 & 79.185 & 339.241 & 0.02171 & 0.86 & 0.48 & 0.01991 & 0.927 & 20083 \\
\hline 6082 & 5.5218 & 79.157 & 339.358 & 0.02175 & 1.11 & 0.60 & 0.02519 & 1.161 & 25109 \\
\hline 6083 & 5.5219 & 79.125 & 339.487 & 0.02177 & 0.95 & 0.67 & 0.03110 & 1.418 & 30609 \\
\hline 6084 & 5.5218 & 79.089 & 339.618 & 0.02175 & 0.83 & 0.54 & 0.03763 & 1.699 & 36611 \\
\hline 6085 & 5.5219 & 79.049 & 339.774 & 0.02173 & 0.99 & 0.43 & 0.04477 & 2.001 & 43020 \\
\hline 6086 & 5.5219 & 79.009 & 339.942 & 0.02166 & 0.68 & 0.09 & 0.05254 & 2.328 & 49920 \\
\hline 6087 & 5.5218 & 78.965 & 340.106 & 0.02164 & 1.03 & -0.04 & 0.06092 & 2.670 & 57107 \\
\hline 6088 & 5.5219 & 78.913 & 340.301 & 0.02154 & 0.82 & -0.54 & 0.06993 & 3.040 & 64822 \\
\hline 6098 & 4.8343 & 69.270 & 339.143 & 0.02138 & 1.49 & 0.14 & 0.01524 & 0.731 & 12075 \\
\hline 6099 & 4.8342 & 69.246 & 339.248 & 0.02142 & 1.13 & 0.27 & 0.01991 & 0.947 & 15621 \\
\hline 6100 & 4.8341 & 69.214 & 339.376 & 0.02143 & 0.92 & 0.31 & 0.02519 & 1.189 & 19576 \\
\hline 6101 & 4.8341 & 69.186 & 339.505 & 0.02152 & 0.86 & 0.67 & 0.03110 & 1.451 & 23842 \\
\hline 6102 & 4.8341 & 69.154 & 339.648 & 0.02155 & 1.14 & 0.78 & 0.03762 & 1.739 & 28507 \\
\hline 6103 & 4.8341 & 69.118 & 339.810 & 0.02156 & 1.33 & 0.80 & 0.04477 & 2.050 & 33527 \\
\hline 6104 & 4.8340 & 69.082 & 339.973 & 0.02154 & 1.68 & 0.68 & 0.05254 & 2.386 & 38917 \\
\hline 6116 & 4.1414 & 59.263 & 339.161 & 0.02069 & 1.87 & -2.02 & 0.01525 & 0.760 & 9130 \\
\hline 6117 & 4.1415 & 59.243 & 339.262 & 0.02103 & 1.17 & -0.37 & 0.01991 & 0.971 & 11658 \\
\hline 6118 & 4.1415 & 59.219 & 339.391 & 0.02109 & 0.97 & -0.14 & 0.02520 & 1.220 & 14608 \\
\hline 6119 & 4.1416 & 59.195 & 339.521 & 0.02113 & 0.71 & 0.05 & 0.03110 & 1.494 & 17856 \\
\hline 6120 & 4.1417 & 59.167 & 339.668 & 0.02124 & 1.23 & 0.54 & 0.03763 & 1.786 & 21312 \\
\hline 6121 & 4.1415 & 59.135 & 339.834 & 0.02131 & 1.51 & 0.81 & 0.04478 & 2.105 & 25051 \\
\hline 6133 & 3.4497 & 49.312 & 339.056 & 0.02065 & 1.89 & -1.00 & 0.01120 & 0.564 & 4664 \\
\hline 6134 & 3.4499 & 49.296 & 339.158 & 0.02069 & 1.49 & -0.82 & 0.01525 & 0.763 & 6306 \\
\hline 6135 & 3.4499 & 49.280 & 339.273 & 0.02069 & 0.93 & -0.87 & 0.01991 & 0.994 & 8195 \\
\hline 6136 & 3.4498 & 49.256 & 339.405 & 0.02070 & 0.90 & -0.85 & 0.02519 & 1.252 & 10305 \\
\hline 6137 & 3.4499 & 49.236 & 339.544 & 0.02079 & 1.02 & -0.42 & 0.03110 & 1.532 & 12586 \\
\hline 6138 & 3.4499 & 49.212 & 339.704 & 0.02082 & 1.09 & -0.32 & 0.03763 & 1.843 & 15102 \\
\hline
\end{tabular}


Table 3. Thermal conductivity of argon, steady-state method-Continued

\begin{tabular}{|c|c|c|c|c|c|c|c|c|c|}
\hline $\begin{array}{l}\text { Point } \\
\text { no. }\end{array}$ & $\begin{array}{c}p \\
(\mathrm{MPa})\end{array}$ & $\begin{array}{c}\rho \\
\left(\mathrm{kg} \mathrm{m}^{-3}\right)\end{array}$ & $\begin{array}{l}T_{\exp } \\
(\mathrm{K})\end{array}$ & $\begin{array}{c}\lambda_{\exp } \\
\left(\mathrm{W} \mathrm{m}^{-1} \mathrm{~K}^{-1}\right)\end{array}$ & $T B A N D$ & $\begin{array}{l}\text { Relative } \\
\text { dev. (\%) }\end{array}$ & $\begin{array}{c}q \\
\left(\mathrm{~W} \mathrm{~m}^{-1}\right)\end{array}$ & $\Delta T$ & $R a$ \\
\hline \multicolumn{10}{|c|}{ Nominal temperature $340 \mathrm{~K}$} \\
\hline 6139 & 3.4501 & 49.192 & 339.859 & 0.02091 & 1.43 & 0.06 & 0.04477 & 2.173 & 17771 \\
\hline 6140 & 3.4499 & 49.160 & 340.041 & 0.02097 & 1.57 & 0.29 & 0.05255 & 2.531 & 20635 \\
\hline 6152 & 3.4481 & 49.272 & 339.157 & 0.02064 & 1.18 & -1.09 & 0.01525 & 0.765 & 6316 \\
\hline 6153 & 3.4481 & 49.252 & 339.278 & 0.02066 & 1.16 & -1.00 & 0.01991 & 0.995 & 8196 \\
\hline 6154 & 3.4481 & 49.232 & 339.408 & 0.02068 & 0.64 & -0.92 & 0.02520 & 1.253 & 10302 \\
\hline 6155 & 3.4481 & 49.212 & 339.550 & 0.02079 & 0.76 & -0.42 & 0.03110 & 1.532 & 12572 \\
\hline 6156 & 3.4483 & 49.188 & 339.706 & 0.02081 & 0.86 & -0.40 & 0.03763 & 1.844 & 15100 \\
\hline 6157 & 3.4483 & 49.164 & 339.869 & 0.02090 & 1.32 & 0.02 & 0.04478 & 2.175 & 17759 \\
\hline 6158 & 3.4483 & 49.136 & 340.042 & 0.02102 & 2.00 & 0.53 & 0.05254 & 2.525 & 20568 \\
\hline 6170 & 2.7656 & 39.461 & 339.156 & 0.02078 & 1.30 & 0.73 & 0.01525 & 0.763 & 4010 \\
\hline 6171 & 2.7655 & 39.445 & 339.283 & 0.02044 & 1.12 & -0.97 & 0.01991 & 1.011 & 5301 \\
\hline 6172 & 2.7657 & 39.429 & 339.424 & 0.02044 & 0.73 & -0.98 & 0.02519 & 1.276 & 6677 \\
\hline 6173 & 2.7658 & 39.413 & 339.566 & 0.02045 & 0.68 & -0.96 & 0.03110 & 1.570 & 8199 \\
\hline 6174 & 2.7658 & 39.393 & 339.727 & 0.02048 & 0.75 & -0.86 & 0.03763 & 1.891 & 9854 \\
\hline 6175 & 2.7657 & 39.369 & 339.889 & 0.02059 & 1.30 & -0.37 & 0.04477 & 2.232 & 11599 \\
\hline 6176 & 2.7658 & 39.349 & 340.086 & 0.02062 & 1.14 & -0.26 & 0.05254 & 2.606 & 13507 \\
\hline 6177 & 2.7658 & 39.325 & 340.282 & 0.02070 & 1.70 & 0.07 & 0.06093 & 3.000 & 15503 \\
\hline 6178 & 2.7659 & 39.301 & 340.493 & 0.02076 & 1.60 & 0.34 & 0.06993 & 3.419 & 17619 \\
\hline 6180 & 2.0708 & 29.506 & 339.062 & 0.02022 & 1.90 & -0.88 & 0.01120 & 0.579 & 1687 \\
\hline 6181 & 2.0708 & 29.498 & 339.165 & 0.02020 & 1.40 & -0.97 & 0.01524 & 0.788 & 2292 \\
\hline 6182 & 2.0710 & 29.490 & 339.293 & 0.02015 & 1.08 & -1.26 & 0.01991 & 1.030 & 2991 \\
\hline 6183 & 2.0710 & 29.478 & 339.423 & 0.02018 & 0.63 & -1.16 & 0.02519 & 1.300 & 3768 \\
\hline 6184 & 2.0711 & 29.462 & 339.580 & 0.02017 & 0.51 & -1.24 & 0.03110 & 1.602 & 4636 \\
\hline 6185 & 2.0710 & 29.446 & 339.741 & 0.02018 & 0.58 & -1.24 & 0.03763 & 1.935 & 5584 \\
\hline 6186 & 2.0710 & 29.434 & 339.922 & 0.02016 & 0.52 & -1.38 & 0.04477 & 2.300 & 6622 \\
\hline 6187 & 2.0710 & 29.414 & 340.117 & 0.02018 & 0.53 & -1.29 & 0.05254 & 2.690 & 7722 \\
\hline 6188 & 2.0711 & 29.398 & 340.325 & 0.02026 & 0.92 & -0.97 & 0.06092 & 3.101 & 8878 \\
\hline 6189 & 2.0712 & 29.378 & 340.536 & 0.02036 & 1.41 & -0.53 & 0.06993 & 3.535 & 10089 \\
\hline 6190 & 2.0712 & 29.358 & 340.775 & 0.02037 & 1.04 & -0.51 & 0.07955 & 4.009 & 11403 \\
\hline 6206 & 1.3695 & 19.475 & 339.154 & 0.02012 & 1.49 & -0.24 & 0.01525 & 0.793 & 996 \\
\hline 6207 & 1.3695 & 19.467 & 339.277 & 0.02011 & 1.01 & -0.32 & 0.01991 & 1.035 & 1298 \\
\hline 6208 & 1.3696 & 19.459 & 339.418 & 0.02007 & 0.72 & -0.55 & 0.02519 & 1.312 & 1641 \\
\hline 6209 & 1.3696 & 19.451 & 339.566 & 0.02007 & 0.69 & -0.63 & 0.03110 & 1.619 & 2022 \\
\hline 6210 & 1.3696 & 19.443 & 339.742 & 0.02005 & 0.63 & -0.75 & 0.03763 & 1.958 & 2440 \\
\hline 6211 & 1.3696 & 19.431 & 339.926 & 0.02003 & 0.61 & -0.89 & 0.04477 & 2.330 & 2896 \\
\hline 6212 & 1.3696 & 19.419 & 340.130 & 0.02001 & 0.47 & -1.07 & 0.05254 & 2.736 & 3390 \\
\hline 6213 & 1.3696 & 19.407 & 340.348 & 0.02000 & 0.46 & -1.16 & 0.06092 & 3.171 & 3917 \\
\hline 6214 & 1.3696 & 19.391 & 340.575 & 0.01999 & 0.43 & -1.26 & 0.06993 & 3.637 & 4479 \\
\hline 6215 & 1.3696 & 19.379 & 340.835 & 0.01997 & 0.39 & -1.39 & 0.07955 & 4.136 & 5074 \\
\hline 6224 & 0.6978 & 9.903 & 339.155 & 0.01963 & 1.44 & -1.67 & 0.01525 & 0.814 & 262 \\
\hline 6225 & 0.6980 & 9.903 & 339.273 & 0.01996 & 1.01 & -0.04 & 0.01991 & 1.045 & 336 \\
\hline 6226 & 0.6979 & 9.899 & 339.413 & 0.01995 & 0.71 & -0.12 & 0.02519 & 1.323 & 424 \\
\hline 6227 & 0.6980 & 9.895 & 339.567 & 0.01993 & 0.74 & -0.24 & 0.03110 & 1.634 & 523 \\
\hline 6228 & 0.6979 & 9.887 & 339.741 & 0.01992 & 0.54 & -0.33 & 0.03763 & 1.977 & 631 \\
\hline 6229 & 0.6977 & 9.883 & 339.920 & 0.01992 & 0.55 & -0.41 & 0.04477 & 2.353 & 749 \\
\hline 6230 & 0.6977 & 9.875 & 340.129 & 0.01987 & 0.49 & -0.69 & 0.05254 & 2.767 & 878 \\
\hline 6231 & 0.6977 & 9.867 & 340.354 & 0.01986 & 0.50 & -0.80 & 0.06092 & 3.209 & 1015 \\
\hline 6232 & 0.6977 & 9.859 & 340.586 & 0.01990 & 0.50 & -0.64 & 0.06993 & 3.675 & 1158 \\
\hline 6233 & 0.6977 & 9.855 & 340.836 & 0.01989 & 0.46 & -0.76 & 0.07955 & 4.182 & 1314 \\
\hline 6242 & 0.3486 & 4.942 & 339.149 & 0.01970 & 1.77 & -0.79 & 0.01525 & 0.811 & 65 \\
\hline 6243 & 0.3485 & 4.942 & 339.275 & 0.01969 & 0.99 & -0.86 & 0.01991 & 1.060 & 84 \\
\hline 6244 & 0.3487 & 4.942 & 339.406 & 0.01969 & 0.88 & -0.89 & 0.02519 & 1.341 & 107 \\
\hline 6245 & 0.3486 & 4.938 & 339.576 & 0.01969 & 0.54 & -0.92 & 0.03110 & 1.655 & 131 \\
\hline 6246 & 0.3487 & 4.938 & 339.744 & 0.01970 & 0.64 & -0.91 & 0.03763 & 2.001 & 158 \\
\hline 6247 & 0.3487 & 4.934 & 339.933 & 0.01970 & 0.56 & -0.98 & 0.04477 & 2.382 & 188 \\
\hline 6248 & 0.3487 & 4.930 & 340.138 & 0.01969 & 0.52 & -1.04 & 0.05254 & 2.795 & 220 \\
\hline
\end{tabular}


Table 3. Thermal conductivity of argon, steady-state method-Continued

\begin{tabular}{|c|c|c|c|c|c|c|c|c|c|}
\hline $\begin{array}{l}\text { Point } \\
\text { no. }\end{array}$ & $\begin{array}{c}p \\
(\mathrm{MPa})\end{array}$ & $\begin{array}{c}\rho \\
\left(\mathrm{kg} \mathrm{m}^{-3}\right)\end{array}$ & $\begin{array}{l}T_{\text {exp }} \\
(\mathrm{K})\end{array}$ & $\begin{array}{c}\lambda_{\exp } \\
\left(\mathrm{W} \mathrm{m}^{-1} \mathrm{~K}^{-1}\right)\end{array}$ & TBAND & $\begin{array}{l}\text { Relative } \\
\text { dev. }(\%)\end{array}$ & $\begin{array}{c}q \\
\left(\mathrm{~W} \mathrm{~m}^{-1}\right)\end{array}$ & $\Delta T$ & $R a$ \\
\hline \multicolumn{10}{|c|}{ Nominal temperature $340 \mathrm{~K}$} \\
\hline 6249 & 0.3487 & 4.926 & 340.359 & 0.01972 & 0.43 & -0.97 & 0.06092 & 3.237 & 254 \\
\hline 6250 & 0.3486 & 4.922 & 340.601 & 0.01974 & 0.45 & -0.92 & 0.06992 & 3.711 & 290 \\
\hline 6251 & 0.3487 & 4.922 & 340.854 & 0.01974 & 0.38 & -0.99 & 0.07954 & 4.221 & 329 \\
\hline 6260 & 0.1685 & 2.389 & 339.169 & 0.01924 & 1.78 & -2.89 & 0.01525 & 0.830 & 15 \\
\hline 6261 & 0.1685 & 2.389 & 339.292 & 0.01943 & 1.08 & -1.94 & 0.01991 & 1.074 & 20 \\
\hline 6262 & 0.1685 & 2.385 & 339.430 & 0.01954 & 0.64 & -1.41 & 0.02519 & 1.352 & 25 \\
\hline 6263 & 0.1685 & 2.385 & 339.589 & 0.01960 & 0.56 & -1.11 & 0.03110 & 1.663 & 31 \\
\hline 6264 & 0.1685 & 2.385 & 339.760 & 0.01964 & 0.40 & -0.94 & 0.03763 & 2.008 & 37 \\
\hline 6265 & 0.1684 & 2.381 & 339.948 & 0.01966 & 0.33 & -0.90 & 0.04477 & 2.387 & 44 \\
\hline 6266 & 0.1684 & 2.381 & 340.158 & 0.01966 & 0.25 & -0.96 & 0.05254 & 2.801 & 51 \\
\hline 6267 & 0.1684 & 2.377 & 340.381 & 0.01967 & 0.23 & -0.94 & 0.06092 & 3.246 & 59 \\
\hline 6268 & 0.1685 & 2.377 & 340.621 & 0.01968 & 0.16 & -0.98 & 0.06992 & 3.724 & 68 \\
\hline 6269 & 0.1684 & 2.377 & 340.870 & 0.01969 & 0.15 & -0.98 & 0.07955 & 4.235 & 77 \\
\hline
\end{tabular}

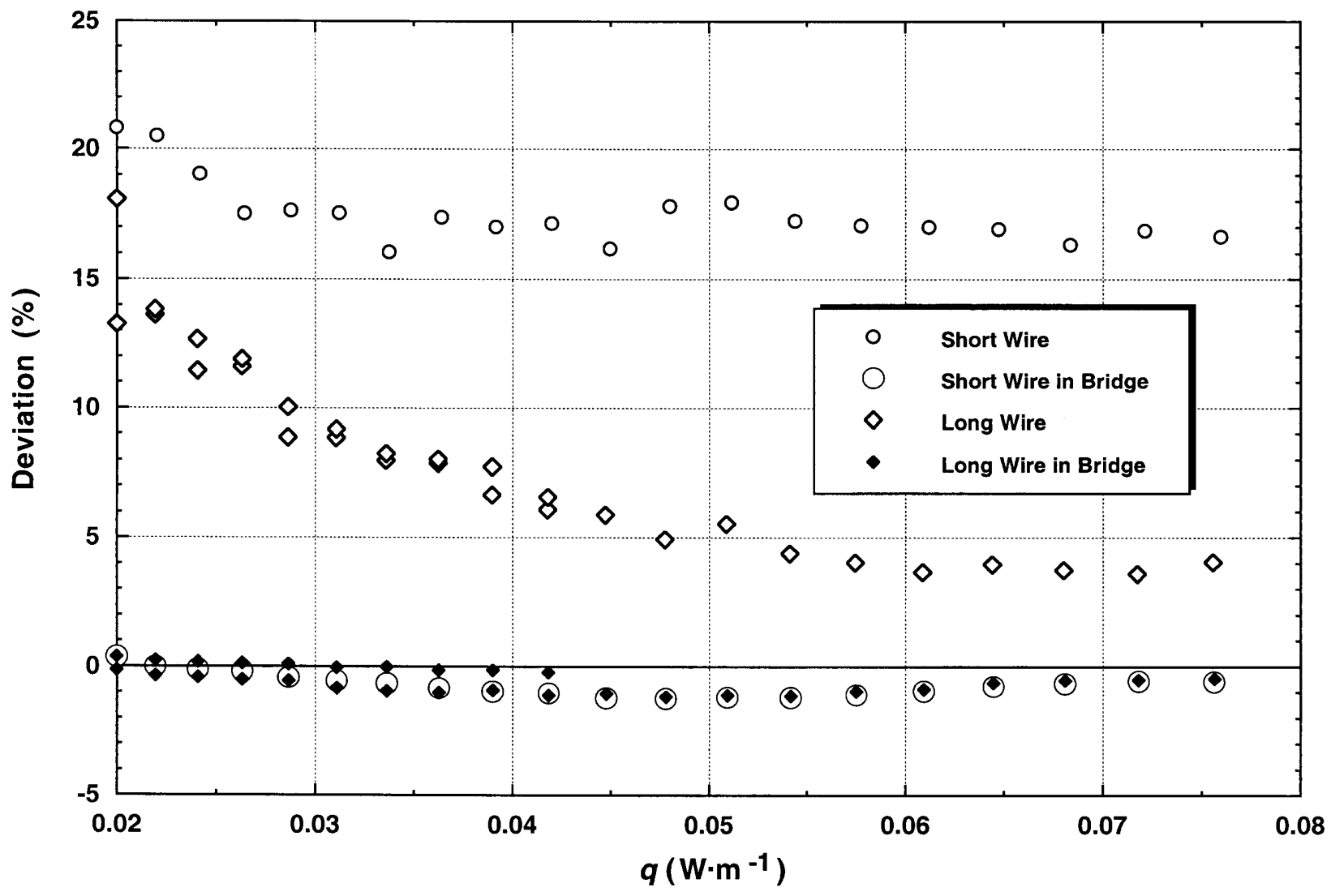

Fig. 11. End effect ratios from "single wire" measurements to determine the effectiveness of end effect compensation by the bridge circuit. The baseline is the correlation of Younglove and Hanley [12]. 
Table 4. Steady-state results with single wires

\begin{tabular}{ccccccccc}
\hline \hline $\begin{array}{c}\text { Point } \\
\text { no. }\end{array}$ & $p$ & $\rho$ & $T_{\text {exp }}$ & $\lambda_{\text {exp }}$ & TBAND & Relative & $q$ \\
$(\mathrm{MPa})$ & $\left(\mathrm{kg} \mathrm{m}^{-3}\right)$ & $(\mathrm{K})$ & $\left(\mathrm{W} \mathrm{m}^{-1} \mathrm{~K}^{-1}\right)$ & & Dev. $(\%)$ & $\left.(\mathrm{W} \mathrm{m})^{-1}\right)$ &
\end{tabular}

\begin{tabular}{|c|c|c|c|c|c|c|c|c|}
\hline \multicolumn{9}{|c|}{ Long hot wire, high pressure } \\
\hline 8001 & 2.0753 & 29.546 & 339.323 & 0.02038 & 1.60 & -0.14 & 0.01991 & 2970 \\
\hline 8001 & 2.0753 & 29.554 & 339.255 & 0.02352 & 0.00 & 13.25 & 0.01989 & 2575 \\
\hline 8002 & 2.0752 & 29.542 & 339.382 & 0.02034 & 1.37 & -0.36 & 0.02195 & 3276 \\
\hline 8002 & 2.0752 & 29.550 & 339.304 & 0.02362 & 0.00 & 13.60 & 0.02193 & 2824 \\
\hline 8003 & 2.0754 & 29.538 & 339.440 & 0.02033 & 1.17 & -0.43 & 0.02409 & 3593 \\
\hline 8003 & 2.0754 & 29.546 & 339.367 & 0.02305 & 0.00 & 11.43 & 0.02407 & 3172 \\
\hline 8004 & 2.0754 & 29.534 & 339.491 & 0.02031 & 1.28 & -0.52 & 0.02633 & 3925 \\
\hline 8004 & 2.0754 & 29.542 & 339.408 & 0.02316 & 0.00 & 11.88 & 0.02631 & 3445 \\
\hline 8005 & 2.0752 & 29.526 & 339.557 & 0.02031 & 0.99 & -0.56 & 0.02867 & 4267 \\
\hline 8005 & 2.0752 & 29.534 & 339.479 & 0.02269 & 0.00 & 10.02 & 0.02864 & 3822 \\
\hline 8006 & 2.0754 & 29.522 & 339.612 & 0.02025 & 0.90 & -0.84 & 0.03111 & 4636 \\
\hline 8006 & 2.0754 & 29.530 & 339.533 & 0.02248 & 0.00 & 9.16 & 0.03108 & 4181 \\
\hline 8007 & 2.0754 & 29.514 & 339.683 & 0.02023 & 0.87 & -0.96 & 0.03364 & 5010 \\
\hline 8007 & 2.0754 & 29.522 & 339.604 & 0.02225 & 0.00 & 8.22 & 0.03361 & 4560 \\
\hline 8008 & 2.0754 & 29.510 & 339.748 & 0.02022 & 0.68 & -1.02 & 0.03628 & 5396 \\
\hline 8008 & 2.0754 & 29.518 & 339.666 & 0.02216 & 0.00 & 7.85 & 0.03624 & 4929 \\
\hline 8009 & 2.0755 & 29.506 & 339.819 & 0.02024 & 0.76 & -0.93 & 0.03901 & 5788 \\
\hline 8009 & 2.0755 & 29.514 & 339.733 & 0.02214 & 0.00 & 7.72 & 0.03898 & 5299 \\
\hline 8010 & 2.0755 & 29.498 & 339.898 & 0.02021 & 0.75 & -1.12 & 0.04185 & 6207 \\
\hline 8010 & 2.0755 & 29.506 & 339.817 & 0.02186 & 0.00 & 6.55 & 0.04181 & 5743 \\
\hline 10001 & 2.0757 & 29.494 & 339.967 & 0.02023 & 0.59 & -1.06 & 0.04478 & 6627 \\
\hline 10001 & 2.0757 & 29.502 & 339.888 & 0.02171 & 0.00 & 5.87 & 0.04474 & 6180 \\
\hline 10002 & 2.0759 & 29.490 & 340.038 & 0.02021 & 0.55 & -1.17 & 0.04781 & 7070 \\
\hline 10002 & 2.0759 & 29.498 & 339.964 & 0.02150 & 0.00 & 4.92 & 0.04776 & 6651 \\
\hline 10003 & 2.0759 & 29.482 & 340.120 & 0.02022 & 0.72 & -1.11 & 0.05095 & 7513 \\
\hline 10003 & 2.0759 & 29.490 & 340.034 & 0.02164 & 0.00 & 5.52 & 0.05090 & 7030 \\
\hline 10004 & 2.0759 & 29.478 & 340.197 & 0.02022 & 0.73 & -1.14 & 0.05417 & 7974 \\
\hline 10004 & 2.0759 & 29.482 & 340.122 & 0.02138 & 0.00 & 4.38 & 0.05412 & 7547 \\
\hline 10005 & 2.0759 & 29.470 & 340.283 & 0.02026 & 0.99 & -0.97 & 0.05750 & 8431 \\
\hline 10005 & 2.0759 & 29.474 & 340.211 & 0.02131 & 0.00 & 4.02 & 0.05745 & 8023 \\
\hline 10006 & 2.0760 & 29.462 & 340.360 & 0.02028 & 1.01 & -0.88 & 0.06093 & 8908 \\
\hline 10006 & 2.0760 & 29.470 & 340.290 & 0.02123 & 0.01 & 3.65 & 0.06087 & 8516 \\
\hline 10007 & 2.0759 & 29.454 & 340.455 & 0.02033 & 1.57 & -0.64 & 0.06446 & 9379 \\
\hline 10007 & 2.0759 & 29.462 & 340.380 & 0.02130 & 0.01 & 3.95 & 0.06440 & 8959 \\
\hline 10008 & 2.0759 & 29.446 & 340.539 & 0.02036 & 1.47 & -0.54 & 0.06809 & 9873 \\
\hline 10008 & 2.0759 & 29.450 & 340.465 & 0.02125 & 0.01 & 3.73 & 0.06802 & 9463 \\
\hline 10009 & 2.0761 & 29.438 & 340.630 & 0.02037 & 1.38 & -0.51 & 0.07181 & 10386 \\
\hline 10009 & 2.0761 & 29.446 & 340.556 & 0.02123 & 0.01 & 3.58 & 0.07174 & 9974 \\
\hline 10010 & 2.0761 & 29.430 & 340.724 & 0.02038 & 1.37 & -0.46 & 0.07564 & 10907 \\
\hline 10010 & 2.0761 & 29.438 & 340.639 & 0.02133 & 0.01 & 4.04 & 0.07557 & 10432 \\
\hline
\end{tabular}

\begin{tabular}{rlrrrrrr}
\hline \multicolumn{7}{c}{ Long hot wire, low pressure } \\
\hline 8001 & 0.1697 & 2.405 & 339.253 & 0.01988 & 1.31 & 0.37 & 0.01991 \\
8001 & 0.1697 & 2.405 & 339.160 & 0.02417 & 0.00 & 18.06 & 0.01989 \\
8002 & 0.1697 & 2.405 & 339.305 & 0.01985 & 1.37 & 0.23 & 0.02195 \\
8002 & 0.1697 & 2.405 & 339.225 & 0.02298 & 0.00 & 13.82 & 0.02193 \\
8003 & 0.1697 & 2.405 & 339.359 & 0.01985 & 1.11 & 0.18 & 0.02409 \\
8003 & 0.1697 & 2.405 & 339.279 & 0.02267 & 0.00 & 12.65 & 0.02406 \\
8004 & 0.1697 & 2.405 & 339.425 & 0.01984 & 0.99 & 0.12 & 0.02633 \\
8004 & 0.1697 & 2.405 & 339.345 & 0.02241 & 0.00 & 11.59 & 0.02630 \\
8005 & 0.1697 & 2.405 & 339.477 & 0.01983 & 0.88 & 0.09 & 0.02866 \\
8005 & 0.1697 & 2.405 & 339.410 & 0.02173 & 0.00 & 8.85 & 0.02863 \\
8006 & 0.1697 & 2.405 & 339.544 & 0.01981 & 0.80 & -0.05 & 0.03110 \\
8006 & 0.1697 & 2.405 & 339.470 & 0.02173 & 0.00 & 8.83 & 0.03107 \\
8007 & 0.1698 & 2.405 & 339.611 & 0.01982 & 0.67 & -0.03 & 0.03364 \\
\hline
\end{tabular}


Table 4. Steady-state results with single wires-Continued

\begin{tabular}{|c|c|c|c|c|c|c|c|c|}
\hline $\begin{array}{c}\text { Point } \\
\text { no. }\end{array}$ & $\begin{array}{c}p \\
(\mathrm{MPa})\end{array}$ & $\begin{array}{c}\rho \\
\left(\mathrm{kg} \mathrm{m}^{-3}\right)\end{array}$ & $\begin{array}{l}T_{\text {exp }} \\
(\mathrm{K})\end{array}$ & $\left(\mathrm{W} \mathrm{m}^{-1} \mathrm{~K}^{-1}\right)$ & $T B A N D$ & $\begin{array}{l}\text { Relative } \\
\text { Dev. }(\%)\end{array}$ & $\begin{array}{c}q \\
\left(\mathrm{~W} \mathrm{~m}^{-1}\right)\end{array}$ & $R a$ \\
\hline \multicolumn{9}{|c|}{ Long hot wire, low pressure } \\
\hline 8007 & 0.1698 & 2.405 & 339.540 & 0.02153 & 0.00 & 7.96 & 0.03360 & 31 \\
\hline 8008 & 0.1696 & 2.401 & 339.682 & 0.01980 & 0.69 & -0.15 & 0.03627 & 36 \\
\hline 8008 & 0.1696 & 2.401 & 339.603 & 0.02155 & 0.00 & 8.01 & 0.03624 & 33 \\
\hline 8009 & 0.1697 & 2.401 & 339.748 & 0.01980 & 0.65 & -0.14 & 0.03901 & 39 \\
\hline 8009 & 0.1697 & 2.401 & 339.678 & 0.02124 & 0.00 & 6.64 & 0.03897 & 36 \\
\hline 8010 & 0.1696 & 2.401 & 339.827 & 0.01979 & 0.48 & -0.23 & 0.04184 & 41 \\
\hline 8010 & 0.1696 & 2.401 & 339.757 & 0.02111 & 0.00 & 6.07 & 0.04180 & 39 \\
\hline
\end{tabular}

\begin{tabular}{|c|c|c|c|c|c|c|c|c|}
\hline \multicolumn{9}{|c|}{ Short hot wire, high pressure } \\
\hline 9001 & 2.0756 & 29.550 & 339.322 & 0.02048 & 1.73 & 0.36 & 0.01991 & 2956 \\
\hline 9001 & 2.0756 & 29.562 & 339.220 & 0.02577 & 0.00 & 20.81 & 0.01999 & 2365 \\
\hline 9002 & 2.0757 & 29.550 & 339.379 & 0.02041 & 1.36 & -0.01 & 0.02195 & 3267 \\
\hline 9002 & 2.0757 & 29.558 & 339.267 & 0.02567 & 0.00 & 20.51 & 0.02204 & 2615 \\
\hline 9003 & 2.0757 & 29.542 & 339.433 & 0.02039 & 1.55 & -0.12 & 0.02409 & 3583 \\
\hline 9003 & 2.0757 & 29.554 & 339.318 & 0.02521 & 0.00 & 19.03 & 0.02419 & 2919 \\
\hline 9004 & 2.0758 & 29.538 & 339.487 & 0.02038 & 1.15 & -0.20 & 0.02633 & 3914 \\
\hline 9004 & 2.0758 & 29.550 & 339.371 & 0.02475 & 0.00 & 17.52 & 0.02644 & 3245 \\
\hline 9005 & 2.0758 & 29.534 & 339.548 & 0.02033 & 1.12 & -0.44 & 0.02867 & 4265 \\
\hline 9005 & 2.0758 & 29.546 & 339.420 & 0.02478 & 0.00 & 17.63 & 0.02878 & 3524 \\
\hline 9006 & 2.0757 & 29.526 & 339.613 & 0.02031 & 1.10 & -0.57 & 0.03110 & 4625 \\
\hline 9006 & 2.0757 & 29.538 & 339.474 & 0.02476 & 0.00 & 17.54 & 0.03123 & 3822 \\
\hline 9007 & 2.0757 & 29.522 & 339.680 & 0.02029 & 0.94 & -0.66 & 0.03364 & 4997 \\
\hline 9007 & 2.0757 & 29.534 & 339.542 & 0.02432 & 0.00 & 16.04 & 0.03377 & 4200 \\
\hline 9008 & 2.0758 & 29.518 & 339.746 & 0.02026 & 0.89 & -0.85 & 0.03628 & 5389 \\
\hline 9008 & 2.0758 & 29.530 & 339.583 & 0.02471 & 0.00 & 17.37 & 0.03642 & 4453 \\
\hline 9009 & 2.0757 & 29.510 & 339.817 & 0.02023 & 0.78 & -0.98 & 0.03901 & 5792 \\
\hline 9009 & 2.0757 & 29.526 & 339.644 & 0.02461 & 0.00 & 17.01 & 0.03917 & 4802 \\
\hline 9010 & 2.0758 & 29.502 & 339.884 & 0.02022 & 0.78 & -1.04 & 0.04185 & 6205 \\
\hline 9010 & 2.0758 & 29.522 & 339.697 & 0.02466 & 0.00 & 17.15 & 0.04202 & 5135 \\
\hline 11001 & 2.0765 & 29.506 & 339.950 & 0.02019 & 0.58 & -1.22 & 0.04478 & 6644 \\
\hline 11001 & 2.0765 & 29.526 & 339.759 & 0.02437 & 0.00 & 16.18 & 0.04496 & 5552 \\
\hline 11002 & 2.0766 & 29.502 & 340.028 & 0.02019 & 0.83 & -1.25 & 0.04781 & 7081 \\
\hline 11002 & 2.0766 & 29.522 & 339.805 & 0.02486 & 0.00 & 17.80 & 0.04801 & 5807 \\
\hline 11003 & 2.0767 & 29.498 & 340.106 & 0.02020 & 0.76 & -1.20 & 0.05094 & 7527 \\
\hline 11003 & 2.0767 & 29.518 & 339.868 & 0.02490 & 0.00 & 17.94 & 0.05115 & 6167 \\
\hline 11004 & 2.0766 & 29.490 & 340.184 & 0.02021 & 0.73 & -1.21 & 0.05417 & 7987 \\
\hline 11004 & 2.0766 & 29.510 & 339.940 & 0.02470 & 0.00 & 17.24 & 0.05439 & 6601 \\
\hline 11005 & 2.0766 & 29.482 & 340.266 & 0.02023 & 0.94 & -1.10 & 0.05750 & 8450 \\
\hline 11005 & 2.0766 & 29.502 & 340.011 & 0.02464 & 0.00 & 17.06 & 0.05774 & 7009 \\
\hline 11006 & 2.0766 & 29.474 & 340.357 & 0.02026 & 1.04 & -0.96 & 0.06093 & 8921 \\
\hline 11006 & 2.0766 & 29.498 & 340.091 & 0.02464 & 0.00 & 17.02 & 0.06118 & 7415 \\
\hline 11007 & 2.0766 & 29.466 & 340.440 & 0.02031 & 1.60 & -0.77 & 0.06446 & 9398 \\
\hline 11007 & 2.0766 & 29.490 & 340.163 & 0.02462 & 0.00 & 16.94 & 0.06472 & 7837 \\
\hline 11008 & 2.0768 & 29.458 & 340.523 & 0.02033 & 1.45 & -0.66 & 0.06808 & 9894 \\
\hline 11008 & 2.0768 & 29.486 & 340.243 & 0.02445 & 0.00 & 16.33 & 0.06836 & 8320 \\
\hline 11009 & 2.0768 & 29.450 & 340.622 & 0.02035 & 1.52 & -0.57 & 0.07181 & 10401 \\
\hline 11009 & 2.0768 & 29.478 & 340.320 & 0.02461 & 0.00 & 16.87 & 0.07211 & 8703 \\
\hline 11010 & 2.0768 & 29.442 & 340.706 & 0.02035 & 1.39 & -0.59 & 0.07563 & 10930 \\
\hline 11010 & 2.0768 & 29.470 & 340.392 & 0.02455 & 0.00 & 16.65 & 0.07595 & 9171 \\
\hline \multicolumn{9}{|c|}{ Voltmeter across the bridge } \\
\hline 7001 & 0.1695 & 2.401 & 339.238 & 0.01977 & 1.19 & -0.18 & 0.01991 & 20 \\
\hline 7001 & 0.1695 & 2.401 & 339.238 & 0.01977 & 0.20 & -0.18 & 0.01991 & 20 \\
\hline 7002 & 0.1696 & 2.405 & 339.289 & 0.01979 & 0.96 & -0.07 & 0.02195 & 22 \\
\hline
\end{tabular}


Table 4. Steady-state results with single wires-Continued

\begin{tabular}{|c|c|c|c|c|c|c|c|c|}
\hline $\begin{array}{l}\text { Point } \\
\text { no. }\end{array}$ & $\begin{array}{c}p \\
(\mathrm{MPa})\end{array}$ & $\begin{array}{c}\rho \\
\left(\mathrm{kg} \mathrm{m}^{-3}\right)\end{array}$ & $\begin{array}{l}T_{\exp } \\
(\mathrm{K})\end{array}$ & $\left(\begin{array}{c}\lambda_{\exp } \\
\left(\mathrm{W} \mathrm{m}^{-1} \mathrm{~K}^{-1}\right)\end{array}\right.$ & $T B A N D$ & $\begin{array}{l}\text { Relative } \\
\text { Dev. (\%) }\end{array}$ & $\begin{array}{c}q \\
\left(\mathrm{~W} \mathrm{~m}^{-1}\right)\end{array}$ & $R a$ \\
\hline
\end{tabular}

\begin{tabular}{|c|c|c|c|c|c|c|c|c|}
\hline \multicolumn{9}{|c|}{ Voltmeter across the bridge } \\
\hline 7002 & 0.1696 & 2.405 & 339.289 & 0.01979 & 0.15 & -0.07 & 0.02195 & 22 \\
\hline 7003 & 0.1697 & 2.405 & 339.346 & 0.01978 & 0.77 & -0.14 & 0.02409 & 24 \\
\hline 7003 & 0.1697 & 2.405 & 339.346 & 0.01978 & 0.14 & -0.14 & 0.02409 & 24 \\
\hline 7004 & 0.1697 & 2.405 & 339.401 & 0.01978 & 1.07 & -0.16 & 0.02632 & 26 \\
\hline 7004 & 0.1697 & 2.405 & 339.401 & 0.01978 & 0.15 & -0.16 & 0.02632 & 26 \\
\hline 7005 & 0.1698 & 2.405 & 339.460 & 0.01978 & 0.89 & -0.18 & 0.02866 & 28 \\
\hline 7005 & 0.1698 & 2.405 & 339.460 & 0.01978 & 0.13 & -0.18 & 0.02866 & 28 \\
\hline 7006 & 0.1697 & 2.405 & 339.531 & 0.01976 & 0.57 & -0.28 & 0.03110 & 31 \\
\hline 7006 & 0.1697 & 2.405 & 339.531 & 0.01976 & 0.12 & -0.28 & 0.03110 & 31 \\
\hline 7007 & 0.1697 & 2.401 & 339.597 & 0.01975 & 0.54 & -0.36 & 0.03363 & 33 \\
\hline 7007 & 0.1697 & 2.401 & 339.597 & 0.01975 & 0.06 & -0.36 & 0.03363 & 33 \\
\hline 7008 & 0.1697 & 2.401 & 339.661 & 0.01976 & 0.57 & -0.34 & 0.03627 & 36 \\
\hline 7008 & 0.1697 & 2.401 & 339.661 & 0.01976 & 0.11 & -0.34 & 0.03627 & 36 \\
\hline 7009 & 0.1697 & 2.401 & 339.733 & 0.01977 & 0.42 & -0.27 & 0.03900 & 39 \\
\hline 7009 & 0.1697 & 2.401 & 339.733 & 0.01977 & 0.11 & -0.27 & 0.03900 & 39 \\
\hline 7010 & 0.1697 & 2.401 & 339.815 & 0.01975 & 0.41 & -0.42 & 0.04184 & 41 \\
\hline 7010 & 0.1697 & 2.401 & 339.815 & 0.01975 & 0.13 & -0.42 & 0.04184 & 41 \\
\hline \multicolumn{9}{|c|}{ Vacuum, short hot wire, very low pressure } \\
\hline 12001 & 0.0013 & 0.020 & 338.819 & 0.00472 & 25.82 & -318.00 & 0.00125 & 0 \\
\hline 12001 & 0.0013 & 0.020 & 338.901 & 0.00297 & 0.14 & -564.60 & 0.00125 & 0 \\
\hline 12002 & 0.0013 & 0.020 & 339.281 & 0.00431 & 2.59 & -358.20 & 0.00498 & 0 \\
\hline 12002 & 0.0013 & 0.020 & 338.941 & 0.00995 & 0.07 & -98.29 & 0.00500 & 0 \\
\hline 12003 & 0.0014 & 0.020 & 340.050 & 0.00426 & 0.90 & -364.60 & 0.01119 & 0 \\
\hline 12003 & 0.0014 & 0.020 & 339.964 & 0.00456 & 0.04 & -334.00 & 0.01124 & 0 \\
\hline 12004 & 0.0015 & 0.020 & 341.128 & 0.00423 & 0.56 & -369.00 & 0.01989 & 0 \\
\hline 12004 & 0.0015 & 0.020 & 340.812 & 0.00488 & 0.03 & -306.40 & 0.01997 & 0 \\
\hline 12005 & 0.0014 & 0.020 & 342.520 & 0.00422 & 0.38 & -371.80 & 0.03105 & 0 \\
\hline 12005 & 0.0014 & 0.020 & 342.110 & 0.00474 & 0.03 & -319.60 & 0.03118 & 0 \\
\hline
\end{tabular}

thermal conductivity surface for argon [12]. The mean of this adjustment is $-0.35 \%$, the maximum is $-1.25 \%$. The next step is to average the results for the various power levels at each pressure. This step gives us 13 or 14 points per isotherm. Theory indicates that the thermal conductivity is a nearly linear function of density at low densities. Since values are now available, measured by two different methods in the same apparatus, it is reasonable to combine both transient and steady-state values into one result. Thus the final step is to obtain averaged straight lines for each isotherm from the experimental results. These averaged straight lines become the basis for deviation plots to assess the accuracy of the present steady-state and transient results.

The averaged thermal conductivities adjusted to nominal isotherm temperatures are given in Table 5 along with the deviations of these values from the straight lines. From Table 5 it is easy to establish that the mean difference between transient and steady-state measurements is about $1 \%$, with the steady-state values nearly always higher. While transient and steady-state values agree to within their combined uncertainties, we cannot exclude the possibility that a systematic difference of about $1 \%$ may exist between the two methods. Our straight-line intercepts are the values of the dilute-gas thermal conductivity $\lambda_{0}$ and the slopes are values of the first density correction $\lambda_{1}$. The coefficients of the lines with their calculated expanded uncertainty at a level of uncertainty of $95 \%$ are given in Table 6 .

The reason that we have used the thermal conductivity surface of Younglove and Hanley [12] in this paper for comparisons, etc., rather than some of the other possible choices, will now become clear. The dilute-gas thermal conductivities of the Younglove and Hanley model [12] are equivalent to the theoretically derived values of Kestin et al. [14]. We conclude from Table 6 that our dilute-gas thermal conductivities are lower than the theoretical values of Kestin et al. [14] as well as those of Aziz [15]. However, they appear to be in better agreement with those of Aziz. Our first density corrections are seen to depend slightly on temperature, unlike the theoretical results, which are constant. They appear 
Table 5. Averaged thermal conductivity of argon, adjusted to even temperature

\begin{tabular}{|c|c|c|c|c|c|}
\hline \multicolumn{3}{|c|}{ Averaged transient data } & \multicolumn{3}{|c|}{ Averaged steady-state data } \\
\hline $\begin{array}{c}\rho \\
\left(\mathrm{kg} \mathrm{m}^{-3}\right)\end{array}$ & $\left(\begin{array}{c}\lambda_{\exp } \\
\left(\mathrm{W} \mathrm{m}^{-1} \mathrm{~K}^{-1}\right)\end{array}\right.$ & $\begin{array}{l}\text { Dev. from } \\
\text { line }(\%)\end{array}$ & $\begin{array}{c}\rho \\
\left(\mathrm{kg} \mathrm{m}^{-3}\right)\end{array}$ & $\left(\mathrm{W} \mathrm{m}^{-1} \mathrm{~K}^{-1}\right)$ & $\begin{array}{c}\text { Dev. from } \\
\text { line }(\%)\end{array}$ \\
\hline \multicolumn{6}{|c|}{ Nominal temperature $300 \mathrm{~K}$} \\
\hline 122.4886 & 0.02067 & 0.09 & 123.395 & 0.02055 & -0.60 \\
\hline 113.6361 & 0.02043 & -0.01 & 114.259 & 0.02046 & 0.06 \\
\hline 102.7463 & 0.02015 & -0.05 & 103.361 & 0.02007 & -0.53 \\
\hline 91.3571 & 0.01984 & -0.19 & 91.868 & 0.02024 & 1.73 \\
\hline 80.7629 & 0.01956 & -0.28 & 81.234 & 0.01991 & 1.42 \\
\hline 69.1979 & 0.01922 & -0.56 & 69.581 & 0.01934 & 0.02 \\
\hline 57.5930 & 0.01892 & -0.63 & 57.909 & 0.01915 & 0.54 \\
\hline 45.8204 & 0.01868 & -0.35 & 46.072 & 0.01865 & -0.55 \\
\hline 33.9558 & 0.01831 & -0.77 & 34.156 & 0.01847 & 0.07 \\
\hline 21.9155 & 0.01800 & -0.85 & 22.027 & 0.01811 & -0.25 \\
\hline 11.1655 & 0.01778 & -0.59 & 11.229 & 0.01802 & 0.74 \\
\hline 6.0162 & 0.01752 & -1.35 & 6.044 & 0.01802 & 1.45 \\
\hline 2.6765 & 0.01781 & 0.76 & 2.689 & 0.01777 & 0.54 \\
\hline
\end{tabular}

\begin{tabular}{|c|c|c|c|c|c|}
\hline \multicolumn{6}{|c|}{ Nominal temperature $320 \mathrm{~K}$} \\
\hline 127.462 & 0.02181 & -0.48 & 128.149 & 0.02202 & 0.40 \\
\hline 115.574 & 0.02146 & -0.65 & 116.452 & 0.02173 & 0.50 \\
\hline 104.588 & 0.02120 & -0.51 & 105.379 & 0.02148 & 0.71 \\
\hline 93.974 & 0.02087 & -0.75 & 94.717 & 0.02116 & 0.54 \\
\hline 83.579 & 0.02057 & -0.88 & 84.178 & 0.02090 & 0.64 \\
\hline 73.472 & 0.02035 & -0.65 & 73.908 & 0.02080 & 1.47 \\
\hline 62.646 & 0.02008 & -0.57 & 62.982 & 0.02041 & 1.01 \\
\hline 52.152 & 0.01982 & -0.49 & 52.480 & 0.02008 & 0.77 \\
\hline 41.654 & 0.01955 & -0.45 & 41.862 & 0.01959 & -0.28 \\
\hline 31.367 & 0.01920 & -0.86 & 31.399 & 0.01931 & -0.29 \\
\hline 20.677 & 0.01889 & -1.02 & 20.785 & 0.01918 & 0.49 \\
\hline 10.227 & 0.01867 & -0.73 & 10.291 & 0.01908 & 1.43 \\
\hline 5.481 & 0.01851 & -0.92 & 5.513 & 0.01879 & 0.58 \\
\hline 2.573 & 0.01868 & 0.41 & 2.593 & 0.01868 & 0.41 \\
\hline \multicolumn{6}{|c|}{ Nominal temperature $340 \mathrm{~K}$} \\
\hline 115.629 & 0.02243 & -0.85 & 116.632 & 0.02301 & 1.57 \\
\hline 108.091 & 0.02223 & -0.82 & 109.046 & 0.02262 & 0.80 \\
\hline 98.332 & 0.02194 & -0.92 & 99.195 & 0.02247 & 1.35 \\
\hline 88.401 & 0.02173 & -0.64 & 89.156 & 0.02190 & 0.05 \\
\hline 78.434 & 0.02144 & -0.71 & 79.077 & 0.02171 & 0.46 \\
\hline 68.579 & 0.02117 & -0.71 & 69.182 & 0.02151 & 0.80 \\
\hline 58.660 & 0.02091 & -0.65 & 59.203 & 0.02111 & 0.23 \\
\hline 48.800 & 0.02065 & -0.60 & 49.228 & 0.02081 & 0.11 \\
\hline 39.061 & 0.02040 & -0.52 & 39.389 & 0.02059 & 0.37 \\
\hline 29.214 & 0.02009 & -0.72 & 29.442 & 0.02023 & -0.05 \\
\hline 19.283 & 0.01977 & -0.96 & 19.431 & 0.02005 & 0.43 \\
\hline 9.811 & 0.01947 & -1.17 & 9.883 & 0.01989 & 0.96 \\
\hline 4.890 & 0.01951 & -0.27 & 4.934 & 0.01971 & 0.74 \\
\hline 2.365 & 0.01971 & 1.10 & 2.381 & 0.01958 & 0.45 \\
\hline
\end{tabular}

to be in better agreement with the values of Rainwater and Friend $[16,17]$ than with those of Bich and Vogel [18]. Good agreement is found with the previous work using the NIST low temperature instrument [19].

Finally, in Fig. 12 the present results are compared with our earlier ones [1,4,19-22], and with those of other authors [23-28] for a temperature of $300 \mathrm{~K}$, where the baseline is the present least-squares fitted line (coefficients in Table 6). The present results are connected by lines to set them off from the others. All other results were shifted to a temperature of $300 \mathrm{~K}$ by using the thermal-conductivity surface of [12]. The largest shift is 
Table 6. Experimental and theoretical dilute-gas thermal conductivity and first-density coefficients ${ }^{\mathrm{a}}$ for argon. Here, $U$ is the expanded uncertainty (coverage factor $k=2$, and thus a 2 standard deviation estimate)

\begin{tabular}{|c|c|c|c|c|c|c|c|c|}
\hline $\begin{array}{c}T \\
(\mathrm{~K})\end{array}$ & $\begin{array}{c}\lambda_{0}(\exp ) \\
\left(\mathrm{W} \mathrm{m}^{-1} \mathrm{~K}^{-1}\right)\end{array}$ & $\begin{array}{c}2 U \\
\left(\mathrm{~W} \mathrm{~m}^{-1} \mathrm{~K}^{-1}\right)\end{array}$ & $\begin{array}{c}\lambda_{0}^{\mathrm{b}} \\
\left(\mathrm{W} \mathrm{m}{ }^{-1} \mathrm{~K}^{-1}\right)\end{array}$ & $\begin{array}{l}\text { Relative } \\
\text { dev. }(\%)\end{array}$ & $\begin{array}{c}\lambda_{0}^{\mathrm{c}} \\
\left(\mathrm{W} \mathrm{m}^{-1} \mathrm{~K}^{-1}\right)\end{array}$ & $\begin{array}{l}\text { Relative } \\
\text { dev. }(\%)\end{array}$ & $\begin{array}{c}\lambda_{0}^{\mathrm{d}} \\
\left(\mathrm{W} \mathrm{m}^{-1} \mathrm{~K}^{-1}\right)\end{array}$ & $\begin{array}{l}\text { Relative } \\
\text { dev. (\%) }\end{array}$ \\
\hline \multicolumn{9}{|c|}{ Dilute-gas thermal conductivity } \\
\hline 300 & 0.01761 & 0.00010 & 0.01761 & 0 & 0.01784 & -1.30 & 0.01772 & -0.62 \\
\hline 320 & 0.01853 & 0.00010 & 0.01871 & -0.92 & 0.01883 & -1.62 & 0.01870 & -0.92 \\
\hline 340 & 0.01943 & 0.00010 & & & 0.01979 & -1.85 & 0.01966 & -1.18 \\
\hline $\begin{array}{c}T \\
(\mathrm{~K})\end{array}$ & $\begin{array}{c}\lambda_{1}(\exp ) \\
\left(\mathrm{W} \mathrm{m}^{-1} \mathrm{~K}^{-1}\right)\end{array}$ & $\begin{array}{c}2 U \\
\left(\mathrm{~W} \mathrm{~m}^{-1} \mathrm{~K}^{-1}\right)\end{array}$ & $\begin{array}{c}\lambda_{1}^{\mathrm{b}} \\
\left(\mathrm{W} \mathrm{m}^{-1} \mathrm{~K}^{-1}\right)\end{array}$ & $\begin{array}{l}\text { Relative } \\
\text { dev. }(\%)\end{array}$ & $\begin{array}{c}\lambda_{1}^{\mathrm{e}} \\
\left(\mathrm{W} \mathrm{m}^{-1} \mathrm{~K}^{-1}\right)\end{array}$ & $\begin{array}{l}\text { Relative } \\
\text { dev. }(\%)\end{array}$ & $\begin{array}{c}\lambda_{1}{ }^{\mathrm{f}} \\
\left(\mathrm{W} \mathrm{m}{ }^{-1} \mathrm{~K}^{-1}\right)\end{array}$ & $\begin{array}{l}\text { Relative } \\
\text { dev. }(\%)\end{array}$ \\
\hline \multicolumn{9}{|c|}{ First-density coefficient } \\
\hline 300 & 0.00099 & 0.00006 & 0.00106 & -7.07 & 0.00105 & -5.74 & 0.00110 & -10.77 \\
\hline 320 & 0.00106 & 0.00006 & 0.00106 & 0 & 0.00104 & 1.79 & 0.00110 & 3.87 \\
\hline 340 & 0.00110 & 0.00007 & & & 0.00104 & 5.71 & 0.00110 & 0.27 \\
\hline
\end{tabular}

${ }^{\mathrm{a}} \lambda_{1}=(\partial \lambda / \partial \rho)_{T} \mathrm{~W} \mathrm{~L} \mathrm{~mol}{ }^{-1} \mathrm{~m}^{-1} \mathrm{~K}^{-1}$

${ }^{\mathrm{b}}$ Ref. [22]

${ }^{\mathrm{c}}$ Ref. [14]

${ }^{\mathrm{d}}$ Ref. [15]

${ }^{\mathrm{e}}$ Refs. [16,17]

${ }^{\mathrm{f}}$ Ref. [18]

around $2.3 \%$, because a few of the original experimental temperatures are as high as $308 \mathrm{~K}$. Figure 12 shows that the present results, including the new steady-state data, are in good agreement with our earlier results [1,4,19-22]. Figure 12 also shows that all of the values assembled here, which include those from transient experiments, steady-state concentric cylinders [24], and steady-state parallel plate systems [23], agree to within $1 \%$, a truly remarkable result. Comparisons made at the other two temperatures, 320 and $340 \mathrm{~K}$, are quite similar to Fig. 12.

\subsection{Uncertainty}

The measurements were deliberately made over a wide range of power levels. For both transient and steady-state results those power levels which were either too small or too large were eliminated. For power levels that are too low, the bridge imbalance becomes comparable to the background noise level and there is significant uncertainty in the measured temperature rises. In general the instruments require a temperature rise of at least $2.5 \mathrm{~K}$ to obtain accurate transient results (STAT $<0.003$ ). With power levels that are too high, curvature is found in the relation for $\Delta T$ vs $\ln (t)$ for the transient measurements, as is typical of convection. The onset of natural convection is also observed in the steady-state measurements as a time-varying steady-state temperature rise. Imposing certain limits on the experimental uncertainty parameter-a maximum STAT of 0.003 for the transient points and a maximum of $2 \%$ in TBAND for the steady-state points-seem to be appropriate restrictions. The uncertainty in transient thermal conductivity data increases at densities below $28 \mathrm{~kg} \mathrm{~m}^{-3}(p=1$ $\mathrm{MPa})$. For valid steady-state measurements of the thermal conductivity, the Rayleigh number must be less than 70000 . With these restrictions it is found that the relative expanded uncertainty of the transient thermal conductivity is $1 \%$ ( $k=2$, see Fig. 9), the uncertainty of the steady-state thermal conductivity is $2 \%(k=2$, see Fig. 10 ), while the agreement between the two methods is $1 \%$ (see Table 5 ). The overall agreement between our present results, our earlier transient results and the results of many other authors is a truly remarkable $1 \%$ (see Fig. 12).

The steady-state results have a greater uncertainty than the transient ones. This can be attributed to a number of factors. First, the steady-state experiment requires an accurate measurement of the temperature rise $\Delta T$ quite similar to the measurement of thermal diffusivity in the transient hot-wire system [20,21]. Second, the steady-state measurement requires accurate determination of the cell geometry: wire radius, cavity radius, eccentricity. Due to the constraints imposed by the onset of convection, the valid temperature rises for steadystate measurements are half those of the transient ones. Table 7 shows the mean temperature rises for both modes of operation. This may serve as a guide for operating hot-wire cells in either the transient or steady-state mode. 


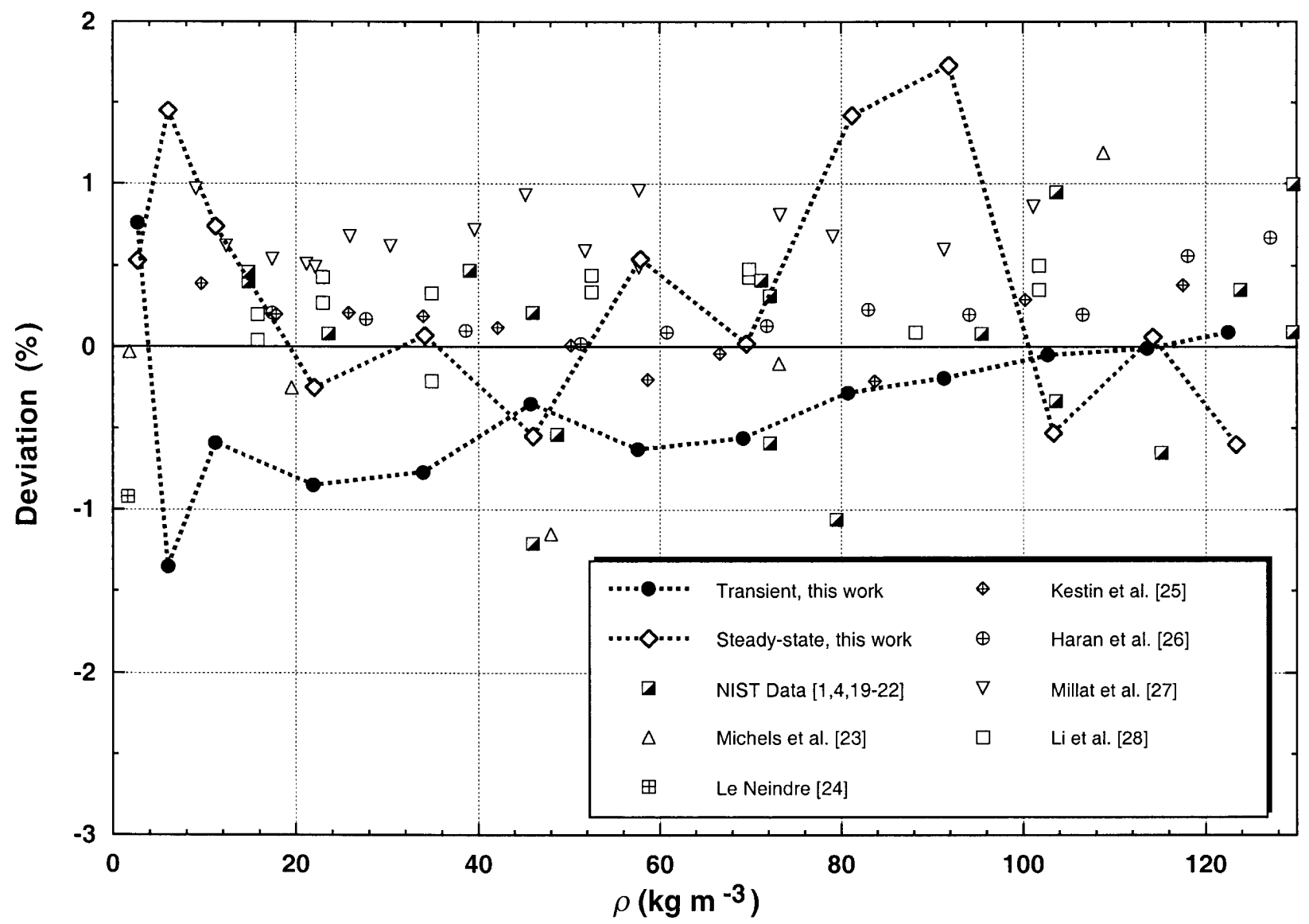

Fig. 12. Deviations of the present results and those of other authors from the line average of the present transient and steady-state results for argon at $300 \mathrm{~K}$.

Table 7. Survey of the valid temperature rises

\begin{tabular}{ccccc}
\hline \hline & \multicolumn{2}{c}{ Transient } & \multicolumn{2}{c}{ Steady state } \\
$T$ & No. points & $\Delta T_{\text {mean }}$ & No. points & $\Delta T_{\text {mean }}$ \\
$(\mathrm{K})$ & & $(\mathrm{K})$ & & $(\mathrm{K})$ \\
& & & & \\
\hline 300 & 98 & 2.082 & 104 & 1.261 \\
320 & 105 & 2.215 & 120 & 1.473 \\
340 & 102 & 3.378 & 119 & 1.826 \\
& & & & \\
\hline
\end{tabular}

\section{Summary}

It has been demonstrated that the thermal conductivity of argon can be measured with a relative expanded uncertainty $(k=2)$ of $2 \%$, using a transient hot-wire system operating in an absolute steady-state mode. The bridge arrangement used in this experiment provides sufficient compensation, eliminating the problem of end effects for both transient and steady-state measurements. The selection of valid steady-state results is based on the shape of the curve of measured voltage rises, on the size of the error in the temperature rise, $T B A N D$, and on the magnitude of the Rayleigh number. Since the fluid gap is quite large in transient hot-wire cells, the steady-state mode is restricted to the low-density gas. The use of the absolute steady-state mode requires no information on the thermophysical properties of the fluid of interest. This is a significant advantage for the measurement of thermal conductivities in the vapor phase of refrigerants where the fluid properties are not known well enough to obtain accurate corrections for transient hot-wire measurements. From a fundamental point of view it can contribute to the determination of accurate pair-interaction potentials for diatomic molecules, especially the long-range weak interactions, a very active research field, by measuring the thermal conductivity of low-temperature vapors. 


\section{References}

[1] H. M. Roder, J. Res. Natl. Bur. Stand. (U.S.) 86, 457 (1981).

[2] H. M. Roder, in Proc. 5th Symposium on Energy and Engineering Sciences, Dept. of Energy (U.S.) CONF-87067 (1987) p.61.

[3] H. M. Roder and R. A. Perkins, J. Res. Natl. Inst. Stand. Technol. 94, 1 (1989).

[4] R. A. Perkins, H. M. Roder, and C. A. Nieto de Castro, J. Res. Natl. Inst. Stand. Technol. 96, 247 (1991).

[5] J. J. Healy, J. J. de Groot, and J. Kestin, Physica 82C, 392 (1976).

[6] M. J. Assael, C. A. Nieto de Castro, H. M. Roder, and W. A. Wakeham, in Measurement of the Transport Properties of Fluids, Experimental Thermodynamics Volume III, W. A. Wakeham, A. Nagashima, and J. V. Sengers, eds., Blackwell Scientific Publications, Oxford (1991) p. 164.

[7] B. Taxis and K. Stephan, Int. J. Thermophys. 15, 141 (1994).

[8] M. J. Assael, L. Karagiannidis, S. M. Richardson, and W. A. Wakeham, Int. J. Thermophys. 13, 223 (1992).

[9] B. Le Neindre and R. Tufeu, in Measurement of the Transport Properties of Fluids, Experimental Thermodynamics Volume III, W. A. Wakeham, A. Nagashima, and J. V. Sengers, eds., Blackwell Scientific Publications, Oxford (1991) Eq (6.1), p. 114.

[10] B. A. Younglove, J. Phys. Chem. Ref. Data 11, Suppl. 1 (1982).

[11] W. J. Taylor and H. L. Johnston, J. Chem. Phys. 14, 219 (1946).

[12] B. A. Younglove and H. J. M. Hanley, J. Phys. Chem. Ref. Data 15, 1323 (1986).

[13] B. Le Neindre and R. Tufeu, in Measurement of the Transport Properties of Fluids, Experimental Thermodynamics Volume III, W. A. Wakeham, A. Nagashima, and J. V. Sengers, eds., Blackwell Scientific Publications, Oxford (1991) Eq. (6.4), p. 137 and Eq. (6.10), p. 140.

[14] J. Kestin, K. Knierim, E. A. Mason, B. Najafi, S. T. Ro, and M. Waldman, J. Phys. Chem. Ref. Data 13, 229 (1984).

[15] R. Aziz, Int. J. Thermophys. 8, 193 (1987).

[16] D. G. Friend and J. C. Rainwater, Chem. Phys. Lett. 107, 590 (1984).

[17] J. C. Rainwater and D. G. Friend, Phys. Rev. A 36, 4062 (1987).

[18] E. Bich and E. Vogel, Int. J. Thermophys. 12, 27 (1991).

[19] C. A. Nieto de Castro and H. M. Roder, J. Res. Natl. Bur. Stand. (U.S.) 86, 293 (1981).

[20] H. M. Roder and C. A. Nieto de Castro, Cryogenics 27, 312 (1987).

[21] H. M. Roder and C. A. Nieto de Castro, in Thermal Conductivity 20, D. P. H. Hasselman, and J. R. Thomas, Jr., eds. Plenum Press, New York (1989) p. 173.

[22] R. A. Perkins, D. G. Friend, H. M. Roder, and C. A. Nieto de Castro, Int. J. Thermophys. 12, 965 (1991).

[23] A. Michels, J. V. Sengers, and L. J. M. van de Klundert, Physica 29, 149 (1963).

[24] B. Le Neindre, Int. J. Heat Mass Transfer 15, 1 (1972).

[25] J. Kestin, R. Paul, A. A. Clifford, and W. A. Wakeham, Physica 100, 349 (1980).

[26] E. N. Haran, G. C. Maitland, M. Mustafa, and W. A. Wakeham, Ber. Bunsenges. Phys. Chem. 87, 657 (1983). [27] J. Millat, M. J. Ross, and W. A. Wakeham, Physica 159A, 28 (1989).

[28] S. F. Y. Li, M. Papadaki, and W. A. Wakeham, in Thermal Conductivity 22, T. W. Tong, ed. Technomic Pub. Co., Inc., Lancaster (1994) p. 531.
About the authors: Hans M. Roder is a physicist retired from the NIST Physical and Chemical Properties Division. Richard A. Perkins and Arno Laesecke are chemical engineers in the NIST Physical and Chemical Properties Division. Carlos A. Nieto de Castro is a chemical engineer, director of the Centro de Ciência e Tecnologia de Materiais and of the Laboratory of Metrology and Testing of the Instituto de Ciência Aplicada e Tecnologia, Faculdade de Ciências da Universidade de Lisboa, and was a Guest Researcher at NIST. The National Institute of Standards and Technology is an agency of the Technology Administration, U.S. Department of Commerce. 